\title{
Modellierung der Feststoffbildung in Einzeltropfen bei der Sprühtrocknung
}

\author{
Dissertation \\ zur \\ Erlangung des Grades \\ Doktor-Ingenieur \\ der \\ Fakultät für Maschinenbau \\ der Ruhr-Universität Bochum
}

von

Philipp Seydel

aus Kiel

Bochum 2005 
Dissertation eingereicht am: 5. Oktober 2005

Tag der mündlichen Prüfung: 10. Januar 2006

Erster Referent: Prof. Dr.-Ing. E. Weidner

Zweiter Referent: Prof. Dr. rer. nat. R. Kümmel 


\section{Inhaltsverzeichnis}

1 Einleitung 11

2 Stand der Technik $\quad 15$

2.1 Allgemeines . . . . . . . . . . . . . . . . . . . . . 15

2.2 Zerstäuber . . . . . . . . . . . . . . . . . . . . . . . 17

2.3 Prozesführung . . . . . . . . . . . . . . . . . . . . . . . . . 18

2.4 Energie- und Massenbilanz . . . . . . . . . . . . . . . . . . . . . . . . . . . 19

2.5 Simulation des Tropfenverhaltens . . . . . . . . . . . . . . . . . . . . . . . . . . . . . . . . . . . . . . .

2.6 Simulation der Sprühtrocknung . . . . . . . . . . . . . . . . . 21

2.7 Berechnung von Verteilungsgrößen mit Populationsbilanzen . . . . . . . . 25

2.8 Problemstellung und Zielsetzung . . . . . . . . . . . . . . . . 25

3 Physikalische Grundlagen der Tropfentrocknung $\quad 27$

3.1 Grundlagen der Trocknung . . . . . . . . . . . . . . . . . . 27

3.1.1 Wärme- und Stoffübergang . . . . . . . . . . . . . . 27

3.1 .2 Verdunstung/Verdampfung . . . . . . . . . . . . . . . 28

3.1 .3 Sattdampfdruck . . . . . . . . . . . . . . . . . . . . 29

3.1.4 Kühlgrenztemperatur . . . . . . . . . . . . . . . . . . . 29

3.1 .5 Trocknung von Tropfen . . . . . . . . . . . . . . . . . . . 31

3.1.6 Genereller Trocknungsverlauf . . . . . . . . . . . . . . . . . 31

3.2 Trocknungsverlauf bei Lösungstropfen . . . . . . . . . . . . . . . . . . 32

3.3 Stabilität des Tropfens und Strömungen im Tropfen . . . . . . . . . . . . . . . 33

3.4 Hüllenwiderstände . . . . . . . . . . . . . . . . . . . . . . 35

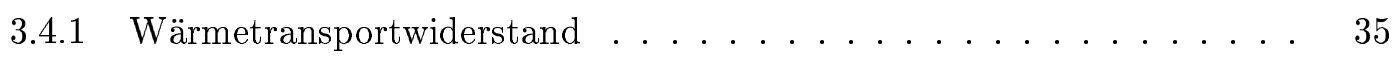

3.4 .2 Stofftransportwiderstand . . . . . . . . . . . . . . . . . 36

4 Grundlagen der Feststoffbildung $\quad 37$

4.1 Übersättigung . . . . . . . . . . . . . . . . . . . . . . . . . . . . . . . 37

4.2 Keimbildung . . . . . . . . . . . . . . . . . . . . . . . . . . 38 
4.3 Kristallwachstum . . . . . . . . . . . . . . . . . . . . . . . . . . . 39

4.4 Kristallisation im Tropfen . . . . . . . . . . . . . . . . . . . . . . 41

5 Mathematisches Modell 43

5.1 Bewegliche Phasengrenzen . . . . . . . . . . . . . . . . . . . . 43

$5.2 \quad$ Stofftransport . . . . . . . . . . . . . . . . . . . . . . . . 44

5.3 Temperatur des Tropfens . . . . . . . . . . . . . . . . . . . . 46

5.4 Modellierung der Feststoffbildung mit Populationsbilanzen . . . . . . . . . . 48

5.4 .1 Partikeldiffusion . . . . . . . . . . . . . . . . . . . 50

5.5 Modellierung des 2. Trocknungsabschnitts . . . . . . . . . . . . . 51

5.5.1 Simulation des mehrschaligen Tropfens mit zwei Koordinatensystemen $\quad 52$

5.5.2 Simulation des mehrschaligen Tropfens in einem Koordinatensystem . 54

5.5.3 Bildung einer Gasblase und Aufblähen des Tropfens . . . . . . . . . 55

6 Numerische Lösung der Differentialgleichungen $\quad 59$

6.1 Lösung der Diffusionsgleichungen . . . . . . . . . . . . . . . . . . . . 59

6.2 Lösung der Populationsbilanzen . . . . . . . . . . . . . . . . . . 60

6.2 .1 Verwendete Diskretisierungsmethode . . . . . . . . . . . . . 62

6.2 .2 Randbedingung am äußeren Tropfenrand . . . . . . . . . . . 65

6.3 Wahl des Lösers . . . . . . . . . . . . . . . . . . . . . . . . . . 65

6.4 Numerische Fehler . . . . . . . . . . . . . . . . . . . . 66

6.5 Numerik bei zwei Koordinatensystemen . . . . . . . . . . . . . 68

6.5.1 Berechnung des Temperaturverlaufs . . . . . . . . . . . . . 69

$\begin{array}{lll}7 & \text { Experimentelles } & \mathbf{7 1}\end{array}$

7.1 Durchgeführte Versuche . . . . . . . . . . . . . . . . . . . . . . . . . 72

7.1 .1 Versuche am Fallturm . . . . . . . . . . . . . . . . . 72

7.1 .2 Tropfen am Draht . . . . . . . . . . . . . . . 75

7.2 Ergebnisse . . . . . . . . . . . . . . . . . 76

7.2 .1 Versuche am Fallturm . . . . . . . . . . . . . . . . . 76

7.2 .2 Tropfen am Draht . . . . . . . . . . . . . . . . . . 82

7.3 Zusammenfassung der Versuche . . . . . . . . . . . . . . . . . 82

8 Ergebnisse der Modellrechnungen $\quad 83$

8.1 Trocknung von Suspensionstropfen . . . . . . . . . . . . . . . 86

8.2 Trocknung von Lösungstropfen . . . . . . . . . . . . . . . . . 88

8.2.1 Einfluß der Trocknungsgastemperatur . . . . . . . . . . . . . . . . 93

8.2.2 Einfluß der Lösungskonzentration . . . . . . . . . . . . . . . . . . . 101 
8.3 Simulation mit gleichzeitiger Bilanzierung des Trocknungsgases . . . . . . 105

8.4 Einfluß der Diskretisierung auf die Partikelanzahldichteverteilung . . . . . . 108

9 Zusammenfassung und Ausblick $\quad 109$

$\begin{array}{ll}\text { A Programmstruktur } & 113\end{array}$

$\begin{array}{ll}\text { Literaturverzeichnis } & \mathbf{1 2 0}\end{array}$ 


\section{Zeichenerklärung}

$\begin{array}{ll}A & \text { Hamaker-Konstante, } \mathrm{J} \\ a_{p} & \text { Partikelabstand, } \mathrm{m} \\ a=\frac{\lambda}{\rho c_{p}} & \text { Temperaturleitkoeffizient, } \mathrm{m} / \mathrm{s} \\ B & \text { Nukleation, } 1 /\left(\mathrm{m}^{3} \mathrm{~s}\right) \\ B_{M} & \text { Spaldingsche Stoffübertragungszahl } \\ C & \text { Konstante } \\ c & \text { Konzentration, } \mathrm{kmol} / \mathrm{m}^{3} \\ c_{p} & \text { Wärmekapazität, } \mathrm{J} /(\mathrm{kg} \mathrm{K}) \\ c_{c} & \text { Clusterkonzentration, } \mathrm{kmol} / \mathrm{m}^{3} \\ c_{m} & \text { Konzentration metastabile Zone, } \mathrm{kmol} / \mathrm{m}^{3} \\ c_{s} & \text { Sättigungskonzentration, } \mathrm{kmol} / \mathrm{m}^{3} \\ c^{*} & \text { Löslichkeit, } k m o l / \mathrm{m}^{3} \\ D & \text { Diffusionskoeffizient, } \mathrm{m}^{2} / \mathrm{s} \\ D_{S} & \text { Oberfächendiffusionskoeffizient, } \mathrm{m}^{2} / \mathrm{s} \\ D_{A B} & \text { Volumendiffusionskoeffizient }, \mathrm{m}^{2} / \mathrm{s} \\ d & \text { Durchmesser, } \mathrm{m} \\ d_{p} & \text { Porendurchmesser, } \mathrm{m} \\ d_{m} & \text { Moleküldurchmesser, } \mathrm{m} \\ F & \text { Haftkraft, N } \\ G & \text { Wachstum, } \mathrm{m} / \mathrm{s} \\ g & \text { Erdbeschleunigung, } \mathrm{m} / \mathrm{s}^{2} \\ h_{v} & \text { Verdampfungsenthalpie }, \mathrm{J} / \mathrm{kg} \\ k & \text { Boltzmannkonstante, } \mathrm{J} / \mathrm{K} \\ k_{B} & \text { Keimbildungsfaktor } \\ k_{G} & \text { Wachstumsfaktor } \\ k_{v} & \text { Volumenformfaktor } \\ L & \text { Partikellänge, } \mathrm{m} \\ l & \text { Länge, m } \\ & \end{array}$




$\begin{array}{ll}M & \text { Molmasse, } \mathrm{g} / \mathrm{mol} \\ \dot{m} & \text { Massenstrom, } \mathrm{kg} / \mathrm{s} \\ N & \text { absolute Partikelzahl } \\ n & \text { Partikelanzahldichte }, 1 / \mathrm{m}^{4} \\ n^{*} & \text { Partikelvolumendichte, } 1 / \mathrm{m} \\ \dot{n} & \text { Partikelanzahldichtestrom }, 1 / \mathrm{m}^{3} \mathrm{~s} \\ n_{b} & \text { Exponent } \\ p & \text { Druck, Pa } \\ R & \text { spezielle Gaskonstante, } \mathrm{J} /(\mathrm{kg} \mathrm{K}) \\ \Re & \text { allgemeine Gaskonstante, } \mathrm{J} /(\mathrm{mol} \mathrm{K}) \\ r & \text { Radius, m } \\ S & \text { Übersättigung, } \mathrm{kmol} / \mathrm{kmol} \\ T & \text { Temperatur, K } \\ t & \text { Zeit, s } \\ v & \text { Geschwindigkeit, } \mathrm{m} / \mathrm{s} \\ w & \text { Massenanteil, kg/kg Lösung } \\ X & \text { Feuchtemassenanteil, } \mathrm{kg} / \mathrm{kg}\end{array}$

\section{Griechische Zeichen}

$\begin{array}{ll}\alpha & \text { Wärmeübergangskoeffizient, } \mathrm{W} /\left(\mathrm{m}^{2} \mathrm{~K}\right) \\ \beta & \text { Stoffübergangskoeffizient, } \mathrm{m} / \mathrm{s} \\ \delta & \text { Diffusionskoeffizient, } \mathrm{m}^{2} / \mathrm{s} \\ \epsilon & \text { Volumenanteil der festen Phase }, \mathrm{m}^{3} / \mathrm{m}^{3} \\ \lambda & \text { Wärmeleitfähigkeit, } \mathrm{W} /(\mathrm{m} \mathrm{K}) \\ \eta & \text { dynamische Viskosität, } \mathrm{kg} /(\mathrm{m} \mathrm{s}) \\ \mu & \text { Diffusionswiderstandsfaktor, - } \\ \nu & \text { kinematische Viskosität, } \mathrm{m}^{2} / \mathrm{s} \\ \nu & \text { Stöchiometrischer Koeffizient, - } \\ \rho & \text { Dichte, kg } / \mathrm{m}^{3} \\ \rho^{\prime} & \text { Stoffdichte (Stoffkonstante), } \mathrm{kg} / \mathrm{m}^{3} \\ \sigma & \text { Oberflächenspannung, } \mathrm{N} / \mathrm{m} \\ \sigma_{Z} & \text { Zugspannung, } \mathrm{N} / \mathrm{m}^{2} \\ \sigma & \text { relative Übersättigung } \\ \tau & \text { Schubspannung, } \mathrm{N} / \mathrm{m}^{2}\end{array}$


Hoch- und tiefgestellte Zeichen

$\begin{array}{ll}\text { a } & \text { außen } \\ \text { i } & \text { innen } \\ \text { G } & \text { gelöste Substanz } \\ \text { hom } & \text { homogen } \\ \text { het } & \text { heterogen } \\ \text { K } & \text { Knudsendiffusion } \\ \text { L } & \text { Lösungsmittel } \\ \text { M } & \text { Molare Diffusion } \\ \text { O } & \text { Oberfläche } \\ \text { P } & \text { Pore } \\ \text { Pg } & \text { Phasengrenze } \\ \text { Sat } & \text { Sättigung } \\ \text { s } & \text { Strahl } \\ \text { U } & \text { Umgebung }\end{array}$

Dimensionslose Kennzahlen

$\mathrm{Nu} \quad$ Nusselt-Zahl

Oh Ohnesorg-Zahl

Pr Prandtl-Zahl

Sc Schmidt-Zahl

Sh Sherwood-Zahl

Re Reynolds-Zahl

We Weber-Zahl 


\section{Kapitel 1}

\section{Einleitung}

Bei der Sprühtrocknung handelt es sich um ein seit etwa 100 Jahren bekanntes Verfahren zur Erzeugung von Pulvern. Eine Flüssigkeit wird in einem heißen Gasstrom zerstäubt, und aus dem feinen Tröpfchennebel entsteht ein Pulver. Bereits Anfang des letzten Jahrhunderts hat es weite Verbreitung in verschiedenen Industrien gefunden. Die Einsatzgebiete liegen von der chemischen über die pharmazeutische bis hin zur Lebensmittelindustrie. Ein Vorteil der Sprühtrocknung besteht darin, daß es sich um einen schonenden Prozeß handelt, d. h. auch temperaturempfindliche Stoffe können getrocknet werden, weil die Tropfentemperatur die Siedetemperatur des Lösungsmittels nicht überschreiten kann. Darüber hinaus besitzen die für den Prozeß verwendeten Apparate einen relativ einfachen Aufbau mit nur wenigen mechanisch bewegten Teilen. Ein weiterer Vorteil gegenüber anderen Verfahren ist zudem, daß keine weiteren Prozeßschritte im Anschluß an die Sprühtrocknung mehr notwendig sind. Demgegenüber stehen allerdings relativ hohe Energiekosten zur Verdampfung des Lösungsmittels. Auch ist bei Verwendung organischer Lösungsmittel umfangreicher Explosionsschutz notwendig.

Es werden je nach Prozeßführung verschiedene Sprühtrocknertypen unterschieden: Die flüssige Lösung kann im Gleichstrom oder im Gegenstrom zur Strömungsrichtung des Trocknungsgases getrocknet werden. Ein wichtiges Element ist der Zerstäuber. Je nach Zerstäubertyp können bestimmte Tropfengrößen und Verteilungsbreiten erreicht werden. Tropfen können durch Zentrifugal- oder Druckkräfte, kinetische oder Schallenergie erzeugt werden.

Die Wahl des Trockners und die Steuerung der Prozesse basieren bisher jedoch noch immer hauptsächlich auf Empirie. Es gibt bislang noch keine ausreichende theoretische oder physikalische Basis für die Auslegung von Trocknern und die Steuerung der Prozesse. Vor dem Hintergrund steigender Produktanforderungen sind detaillierte Kenntnisse der mikroskopischen Feststoffbildungsprozesse jedoch notwendig, um Produkte mit neuen Eigenschaften entwickeln zu können. Produkteigenschaften sind hier Staubneigung, Rieselfähigkeit, Löslichkeit oder Freisetzungsrate. Diese können u.a. mittels Einflußnahme auf Partikelgrößenverteilung, Kri- 
stallmodifikation, Grenzflächenspannung der Flüssigkeit oder Partikelform verändert werden [1].

Beim Sprühtrocknungsprozeß gilt es, drei Bereiche zu modellieren: Die Gasphase und die Bewegung der Tröpfchen im Trocknungsraum, die Zerstäubung der Flüssigkeit und die trocknenden Tropfen. Zur Simulation der Gasphase sowie der Zerstäubung kann kommerzielle CFD-Software (Computational Fluid Dynamics) verwendet werden. Für die Beschreibung der sich bildenden Festphase in den Tropfen zur Vorhersage der mikroskopischen Struktur der einzelnen Pulverpartikel und damit der Pulvereigenschaften muß eigens ein neues Modell entwickelt werden. Die prinzipiell bei der Sprühtrocknung auftretenden Morphologien sind durch verschiedene Untersuchungen in den vergangenen Jahren bekannt. Ziel einer Modellierung muß es sein, sowohl die Verteilung des Feststoffs als auch weitere Eigenschaften des Feststoffs im trocknenden Tropfen vorhersagen zu können. In den letzten Jahrzehnten haben sich bereits einige Arbeitsgruppen mit der Entwicklung solcher Modelle befaßst und diese haben im Laufe der Jahre mit zunehmender Computerleistung an Komplexität gewonnen. In den meisten Fällen wird die Festphasenbildung im Tropfen jedoch mit sehr einfachen Modellen beschrieben. Die Erstarrungsprozesse, bei denen der Feststoff eine kristalline oder amorphe Struktur erhält, werden in der Regel nicht modelliert. Allerdings ist bei der Modellierung des Sprühtrocknungsprozesses ein großes Problem, daß eine physikalisch-chemische Datenbasis für die Feststoffbildung im mikroskopischen Bereich kaum verfügbar ist. Darüber hinaus werden in der Regel Stoffgemische versprüht, deren physikalisches Verhalten komplex und daher entsprechend schwierig zu beschreiben ist.

Ein geeignetes Mittel zur Beschreibung der Feststoffverteilung im Tropfen sind Populationsbilanzen, mit denen sich Verteilungsgrößen berechnen lassen. Mit ihnen können die Verteilung von Feststoffpartikeln im trocknenden Tropfen und die Eigenschaften dieser Partikel berechnet werden. Mit der Kenntnis der Größe und Verteilung der Feststoffpartikel im Tropfen kann dann auch die Struktur und Dicke einer sich bildenden Hülle beschrieben werden und damit auch ihre Festigkeit. Dies ist wichtig, weil das häufig auftretende Platzen von Hohlpartikeln bei der Sprühtrocknung teilweise unerwünscht ist. Die Populationsbilanzen müssen gekoppelt mit der Massen- und Energiebilanz gelöst werden, um die Schrumpfungsgeschwindigkeit des Tropfens und die Verteilung gelöster Substanzen im Tropfen berechnen zu können. Die Berechnung der Temperaturverteilung im Tropfen ist insbesondere wichtig, sobald sich eine Hülle gebildet hat und sich Gas im Tropfen bildet.

Das Hauptanliegen dieser Arbeit ist, ein besseres Verständnis der mikroskopischen, physikalischen Vorgänge während der Trocknung eines Lösungs- oder Suspensionstropfens zu gewinnen. Dazu soll ein Simulationsprogramm entwickelt werden, welches die Feststoffbildung im Tropfen detailliert mit Hilfe von Populationsbilanzen simuliert. 
Im folgenden wird zunächst der Stand der Technik geschildert. Daran anschließend werden die Grundlagen der Trocknungstechnik sowie der Kristallisation erläutert. Schließlich werden das mathematische Modell beschrieben sowie die verwendeten numerischen Verfahren zur Lösung der Gleichungssysteme. In den abschließenden Kapiteln werden die Ergebnisse der Simulationsrechnungen sowie der Experimente dargestellt. 


\section{Kapitel 2}

\section{Stand der Technik}

\subsection{Allgemeines}

Abbildung 2.1 zeigt das prinzipielle Schema des Sprühtrocknungsprozesses. Eine Lösung oder Suspension wird mit einer Düse in einen heißen Gasstrom zerstäubt. Aus den feinen Tröpfchen (Größe: in der Regel 5 bis $600 \mu \mathrm{m}$ ) entstehen während des Fluges durch den Trocknungsraum durch Verdunstung/Verdampfung der Flüssigkeit Partikel. Die Partikel verlassen den Trocknungsraum gemeinsam mit dem Gas und werden in einem Zyklon vom Gasstrom abgetrennt. Sehr feine Partikel werden anschließend in einem nachgeschalteten Filter aus dem Gas entfernt. Haben die Partikel bereits die gewünschten Eigenschaften, ist keine weitere Behandlung notwendig. Andernfalls müssen weitere Prozesse nachgeschaltet werden.

Die Auslegung von Sprühtrocknern beruht zum derzeitigen Stand der Technik hauptsächlich auf empirisch gewonnenen Daten und der Bilanzierung der Massen- und Energieströme über den Trocknungsraum. Die physikalischen und chemischen Eigenschaften des Eduktes und die Anforderungen an das Produkt bestimmen die Auslegung des Sprühtrockners. Zu den Eigenschaften des Eduktes gehören

- Temperaturstabilität,

- Dichte,

- Feststoffgehalt,

- Viskosität der Lösung bzw. Suspension,

- Art des Lösungsmittels und

- Grenzflächenspannung.

Die Eigenschaften des Edukts bestimmen auch das zu verwendende Trocknungsgas. Die Anforderungen an das Produkt werden bestimmt u.a. durch die 


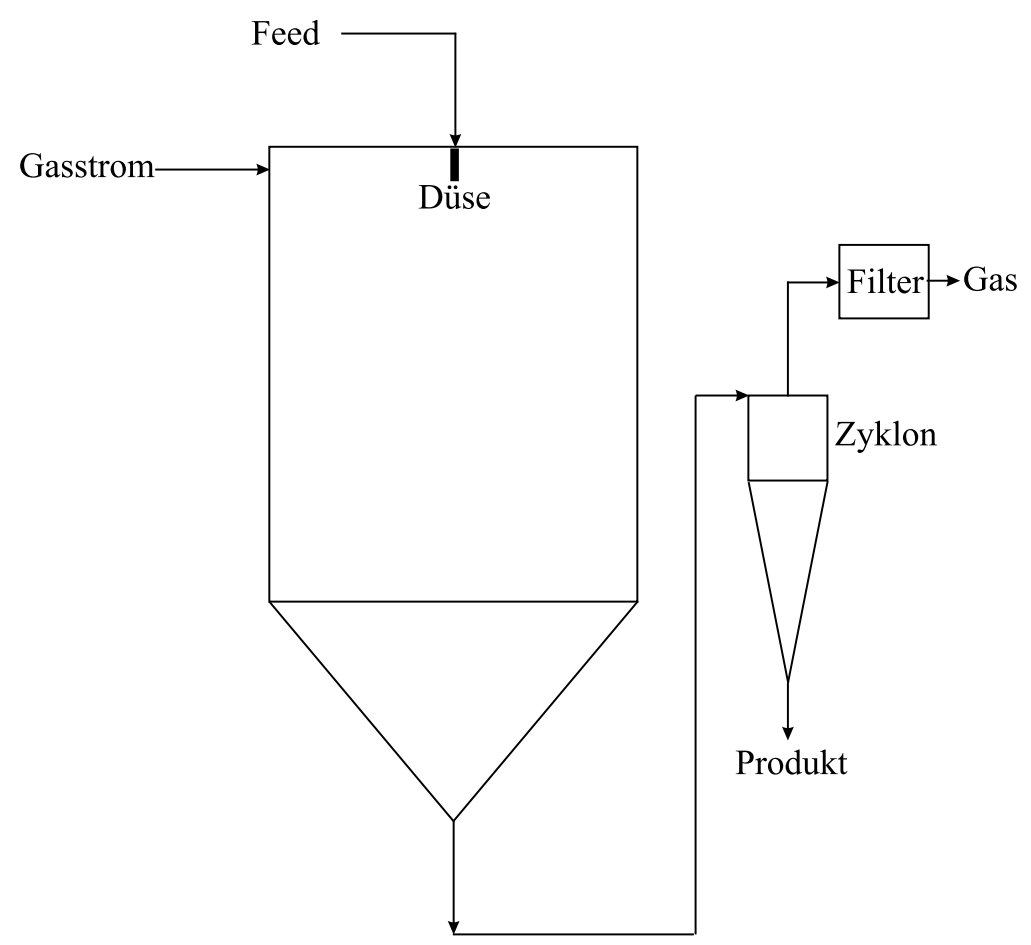

Abbildung 2.1: Aufbau eines Sprühtrocknungsprozesses

- Löslichkeit

- Festigkeit

- Fließ-, Rieselfähigkeit

- Staubneigung

- effektive Dichte

- Schüttdichte

- Restfeuchte

Durch die Wahl der Trocknungsbedingungen und der Zerstäubungsparameter kann Einfluß auf diese Eigenschaften genommen werden (Abb. 2.2). Ohne die mikroskopischen Vorgänge im Detail zu kennen, können die funktionalen Zusammenhänge zwischen Produkteigenschaften und Prozeßparametern jedoch nur empirisch ermittelt und qualitativ beschrieben werden.

Durch die Variation der Eingangs- und Ausgangstemperaturen, des Trocknungsgasmassenstroms und des Eduktmassenstroms können Feuchtigkeit und Dichte des Endprodukts beeinflußt werden. Die Staubneigung wird hauptsächlich durch die Partikelgröße beeinflußt, welche sich durch die Wahl des Zerstäubers und der Zerstäubungsbedingungen steuern läßst. 


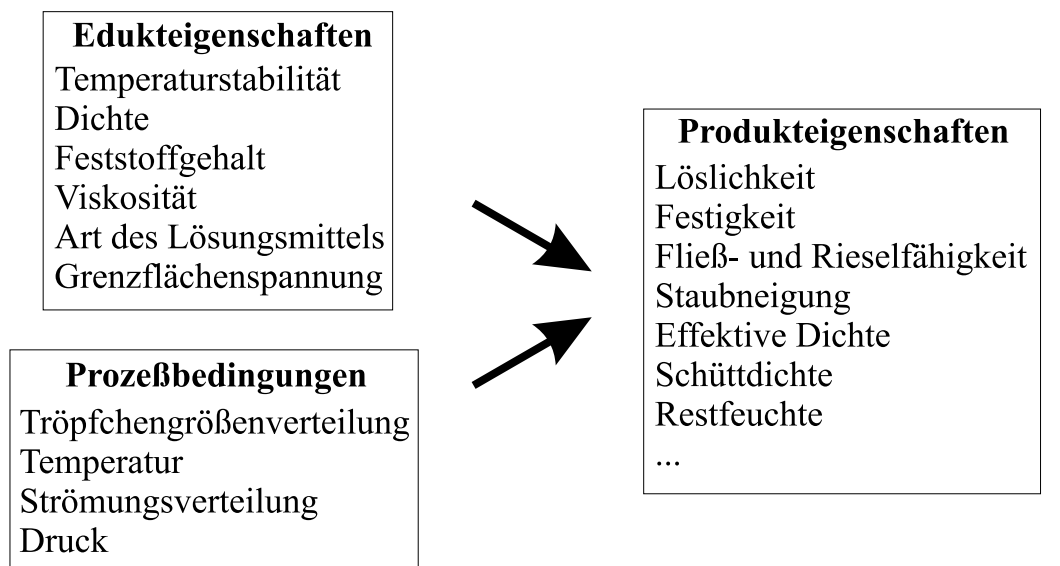

Abbildung 2.2: Die Produkteigenschaften werden von den Eigenschaften des Eduktes und den Prozeßbedingungen beeinflußt.

Die Festigkeit der Partikel hängt von der Dicke und Struktur der Feststoffhülle der einzelnen Partikel ab, was durch die Wahl der Trocknungsgastemperatur beeinflußt wird. Zur Steuerung der Schüttdichte sind das Verhältnis von Feedstrom und Trocknungsgasstrom sowie die Trocknungsgastemperatur geeignet, weil durch diese Parameter die Partikelgröße des Produktes beeinflußt wird. Darüber hinaus hängt die Schüttdichte von der Breite der Partikelgrößenverteilung ab. Je breiter sie ist, desto größer ist die Schüttdichte. Die effektive Dichte ergibt sich aus dem Verhältnis von Durchmesser zur Dicke der Feststoffhülle der einzelnen Partikel. Das Verhältnis kann hauptsächlich über die Trocknungsgastemperatur beeinflußst werden. Je größer die Partikel sind, desto besser ist ihre Fließ- und Rieselfähigkeit, weil das Verhältnis von Haftkraft zu Gewichtskraft bei großen Partikeln geringer ist und die großen Partikel eine geringere relative Oberfläche haben, so daß die zwischen ihnen wirkenden Kräfte geringer sind [2]. Die Partikelgröße wird durch die Zerstäubung beeinflußt [3, 4].

\subsection{Zerstäuber}

Der Zerstäuber, der für die Erzeugung der Tröpfchen verwendet wird, ist der Haupteinflußfaktor für die Partikelgrößenverteilung des Produkts. Die Eigenschaften des Edukts beeinflussen wiederum die Wahl des Zerstäubers, weil nicht jeder Zerstäuber für alle Flüssigkeiten geeignet ist. Bei hoher Viskosität oder hohem Feststoffgehalt kann es zur Verstopfung und zu starkem Abrieb kommen. Zur Sprayerzeugung werden

- Druckdüsen

- Zweistoffdüsen

- Rotationszerstäuber 
- Ultraschallzerstäuber und

- elektrostatische Zerstäuber

verwendet, wobei die Tropfen jeweils aufgrund unterschiedlicher Kräfte entstehen. Bei der Druckdüse strömt die Flüssigkeit in einer turbulenten Strömung mit hohem Druck aus der Düse. Durch die Reibung mit der Luft und durch in der Düse erzeugte Störungen zerfällt der Strahl in Tropfen. Im Fall von Zweistoffdüsen wird die Flüssigkeit durch ein Gas am Düsenaustritt zerstäubt. Bei Rotationszerstäubern dreht sich eine Scheibe bzw. ein Rad, das von der Flüssigkeit überströmt wird bzw. aus dem die Flüssigkeit ausströmt. Durch die Fliehkräfte entstehen aus den Flüssigkeitsstrahlen bzw. dem Flüssigkeitsfilm Tropfen. Bei Ultraschallzerstäubern wirken Schallwellen auf den Flüssigkeitsstrahl, wodurch dieser in Tröpfchen zerfällt. Bei elektrostatischen Zerstäubern wirkt ein statisches elektrisches Feld auf die Austrittsöffnung der Düse. Durch die Zugkräfte wird der Flüssigkeitsstrahl in einzelne Tröpfchen zerstäubt.

Für sehr feine Tröpfchen sind Zweistoffdüsen, Ultraschallzerstäuber sowie elektrostatische Zerstäuber am besten geeignet. Elektrostatische Zerstäuber und Ultraschallzerstäuber werden jedoch nur im Labormaßstab verwendet. Sehr große Tropfen werden mit Rotationszerstäubern oder Druckdüsen erzeugt. Die Tropfengröße hängt im Fall der Rotationszerstäuber von der Rotationsgeschwindigkeit des Zerstäuberrades ab [3, 5].

\subsection{Prozeßführung}

Die Wahl des Trocknungsprozesses hängt davon ab, welches Lösungsmittel eingesetzt werden soll und ob die Partikel nachbehandelt werden müssen:

- offener Kreislauf

- geschlossener Kreislauf

- halb-geschlossener Kreislauf

- keimfrei und hygienisch

- einstufig

- mehrstufig

- Systeme mit Pulveraufbewahrung

Für Prozesse, bei denen wasserlösliche Substanzen mit Luft getrocknet werden sollen, werden offene Kreisläufe verwendet. Wird hingegen mit organischen Lösungsmitteln getrocknet, 


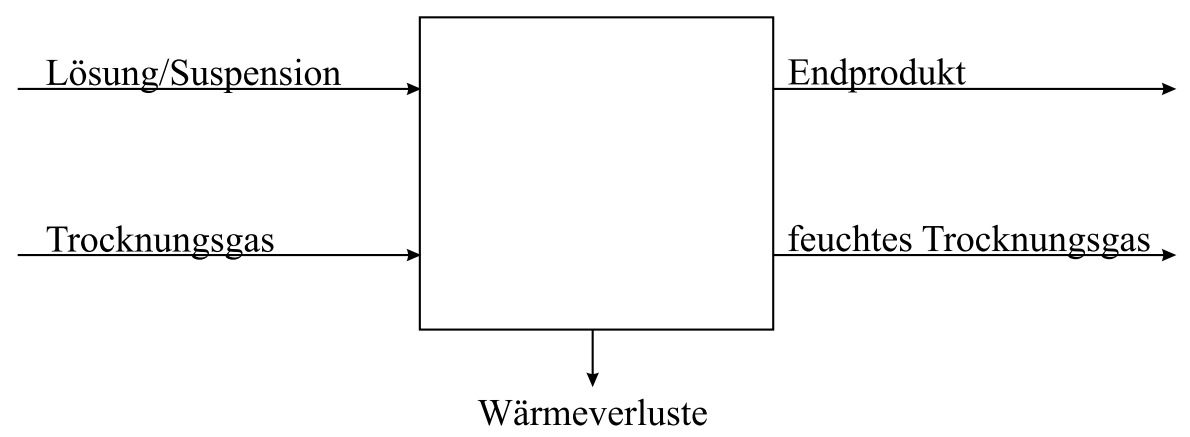

Abbildung 2.3: Bilanz um den Trocknungsraum eines Sprühtrockners

muß ein geschlossener Kreislauf gewählt werden, um eine Umweltbelastung zu vermeiden und das Lösungsmittel zurückgewinnen zu können. Als Übergangsformen gibt es darüber hinaus halb-geschlossene Kreisläufe. In der pharmazeutischen Industrie und Lebensmittelindustrie muß der Prozeß unter keimfreien, hygienischen Bedingungen ablaufen. Machen die Produktanforderungen eine Nachbehandlung der Partikel notwendig, so muß ein mehrstufiger Prozeß verwendet werden. So können in weiteren Schritten die Partikel nachgetrocknet, agglomeriert oder gekühlt werden. Auch eine Energieeffizienzsteigerung ist durch das Nachschalten weiterer Prozesse möglich. Solche Prozesse sind häufig Wirbelschichten oder Bandtrockner [3].

Je nach Produkt werden Gegen- und Gleichstromsprühtrockner sowie Zwischenformen eingesetzt. Das Trocknungsgas wird dabei in oder entgegen der Richtung der Tröpfchen durch den Trocknungsraum geleitet. Bei Gegenstromsprühtrocknern erreicht das Produkt höhere Temperaturen als bei der Gleichstromsprühtrocknung. Daher kann die Gegenstromsprühtrocknung nicht für temperaturempfindliche Stoffe, wie sie beispielsweise in der Lebensmittelindustrie vorkommen, eingesetzt werden. Der Vorteil liegt jedoch in einer höheren Energieeffizienz.

\subsection{Energie- und Massenbilanz}

Aus der Energiebilanz um den Trocknungsraum (Abb. 2.3)

$$
\begin{aligned}
& \underbrace{\dot{m}_{\text {trockene Luft }}\left(c_{p_{\text {Luft ein }}}+X_{\text {ein }} c_{p_{\mathrm{L} \text { Dampf ein }}}\right) T_{\text {ein }}}_{\text {feuchte Luft }}+\underbrace{\dot{m}_{\mathrm{L}_{\text {füssig }}} c_{p_{\mathrm{L} \text { flüssig ein }}} T_{\text {ein }}}_{\text {Lösungsmittel }}+\underbrace{\dot{m}_{\mathrm{G}} c_{p_{\mathrm{G} \text { ein }}} T_{\text {ein }}}_{\text {gelöste Substanz }}= \\
& \underbrace{\dot{m}_{\mathrm{G}} c_{p_{\mathrm{G} \text { aus }}} T_{\text {aus }}}_{\text {Produkt }}+\underbrace{\dot{m}_{\text {trockene Luft }}\left(c_{p_{\text {Luft aus }}}+X_{\text {aus }} c_{p_{\mathrm{L} \text { Dampf aus }}}\right) T_{\text {aus }}}_{\text {feuchte Luft }}+ \\
& \underbrace{\dot{Q}_{\text {Verlust }}} \\
& \text { Wärmeverluste }
\end{aligned}
$$

kann bei bekannter Trocknergeometrie die energetisch erforderliche Trocknungsgasmenge berechnet werden, die zur Trocknung eines bestimmten Massenstroms Edukt notwendig ist. Der 
gesamte Enthalpiestrom, der dem Sprühtrockner zugeführt wird, setzt sich aus dem Enthalpiestrom des heißen Trocknungsgases mit einem bekannten geringen Feuchtegehalt $X_{\text {ein }}$ sowie dem Enthalpiestrom des feuchten Eduktes zusammen. Dabei bezeichnet der Index G die gelöste Substanz und L das Lösungsmittel. Am Ausgang des Trockners ist in der Enthalpiebilanz das trockene Produkt zusammen mit dem feuchten Trocknungsgas zu berücksichtigen. Für die Verdampfung des Lösungsmittels wird dem Trocknungsgas die Verdampfungsenthalpie $h_{v}$ entzogen. Weitere Wärmeverluste $\dot{Q}_{\text {Verlust }}$ treten über die Wände des Trocknungsraumes auf. Als Optimierungsparameter ist der thermische Wirkungsgrad $\eta$ zu nennen, der den Anteil der für die Verdampfung verwendeten Wärme angibt und definiert ist als

$$
\eta=\left(\frac{T_{\text {ein }}-T_{\text {aus }}}{T_{\text {ein }}-T_{\text {Umgebung }}}\right)
$$

Für eine möglichst günstige Nutzung der Energie sollte der Sprühtrockner so ausgelegt werden, daß die Austrittstemperatur möglichst niedrig ist. Bei Temperaturen unter $100^{\circ} \mathrm{C}$ muß der Partialdruck des Lösungsmittels im Trocknungsgas am Austritt gerade eben unterhalb der Sättigungsgrenze liegen, um ein Kondensieren des Lösungsmittels zu verhindern. Bei bekannter Feuchte im Trocknungsgas am Trocknereintritt kann demnach der Gesamtmassenstrom wie folgt berechnet werden:

$$
\dot{m}_{\text {Luft }}=\dot{m}_{\text {trockeneLuft }}+\dot{m}_{\text {trockeneLuft }} \cdot X_{\text {ein }} .
$$

Praktische Erfahrungen zeigen, daß eine Auslegung basierend ausschließlich auf Energie- und Massenbilanz nicht ausreicht, um das gewünschte Produkt zu erhalten. Auch können unerwünschte Ablagerungen an den Wänden des Trocknungsraumes infolge ungünstiger Strömungsverhältnisse auftreten. Erst nach umfangreichen Experimenten kann mit dem Trockner ein Produkt bei gleichbleibender Qualität produziert werden [3].

\subsection{Simulation des Tropfenverhaltens}

Da bei der Sprühtrocknung die entscheidenden Prozesse in den Tropfen ablaufen, ist die Kenntnis des Tropfenverhaltens von großer Bedeutung. In den letzten Jahren sind Fortschritte auf dem Gebiet der Simulation des Tropfenverhaltens gemacht worden [6, 7]. Dazu gehören insbesondere die Vorgänge beim Zusammenprallen von Tropfen [8]. Das Zusammenprallen von Tropfen spielt bei der Sprühtrocknung eine große Rolle, weil es im Trocknungsraum häufig zu Kollisionen von Tropfen kommen kann und dadurch zur Agglomeration [9]. Die Vorgänge beim Zerfallen von Tropfen durch die Wechselwirkung mit dem umgebenden Gas werden bereits seit dem Beginn des letzten Jahrhunderts untersucht [5]. Verschiedene Veröffentlichungen in den letzten Jahren beschäftigen sich mit Transportprozessen in Einzeltropfen in 
Tabelle 2.1: Einzeltropfenmodelle

\begin{tabular}{|l|l|}
\hline Autoren & Modellsystem \\
\hline Sano und Keey (1981) [18] & Suspension \\
Cheong et al. (1986) [19] & Suspension \\
Nešić und Vodnik (1990) [20] & Suspension \\
Lee und Law (1991) [21] & Suspension \\
Bhatia und Sirignano (1992) [22] & Suspension \\
Elperin und Krasovitov (1994) [23] & Suspension \\
Ford (1995) [24] & Lösung \\
Hecht und King (2000) [25] & Lösung \\
Liang et al. (2000) [26] & Suspension \\
Brenn et al. (2001) [27] & Lösung \\
Weiß (2002) [28] & Lösung \\
Kadja und Bergeles (2003) [29] & Suspension \\
Brenn (2004) [30] & Lösung \\
\hline
\end{tabular}

einer umgebenden kontinuierlichen Phase [10, 11]. Darunter sind zahlreiche Modelle, die im Bereich der Verbrennungstechnik entwickelt worden sind und die Verdunstung des Tropfens mitberücksichtigen $[12,13,14,15,16,17]$.

\subsection{Simulation der Sprühtrocknung}

Zur Auslegung von Sprühtrocknern werden zunehmend auch Simulationswerkzeuge verwendet. Die makroskopischen Zustände im Trocknungsraum, wie die Strömung, die Temperaturund Feuchteverteilung sowie die Partikelbahnen, können mit kommerzieller CFD-Software berechnet werden. Zur Beschreibung der mikroskopischen Struktur der Pulver und ihrer Entstehung während des Trocknungsprozesses sind bereits einige Modelle entwickelt worden (Tab. 2.1). Die Modelle sind radialsymmetrisch und eindimensional. Die mikroskopische Struktur des Feststoffs in den einzelnen Partikeln wird jedoch nur in zwei der aufgelisteten Modelle (Ford und Weiß) detaillierter modelliert. Auch der radiale, zeitliche Temperaturverlauf in den Tropfen wird nicht berechnet. Einige der Modelle entstammen auch der Verbrennungstechnik. Da die Vorgänge bei der Verbrennung von Treibstofftröpfchen teilweise den Vorgängen bei der Sprühtrocknung ähneln, sind diese Modelle mit in die Auflistung aufgenommen worden.

Sano und Keey [18]: In diesem Modell wird die Feststoffbildung in Kondensmilchtropfen mit Hilfe von Massenanteilen berechnet. Das Modell kann den zeitlichen radialen Verlauf 
des Massenanteils an Kondensmilch im Tropfen sowie die zeitliche Temperaturänderung im Tropfen berechnen, wobei angenommen wird, daß im Tropfen überall die gleiche Temperatur herrscht. Der Stofftransport findet durch Diffusion statt. Weiterhin kann das Modell das Aufblähen des Partikels aufgrund der Verdampfung der Flüssigkeit im Tropfeninneren simulieren. Die Struktur der Hülle wird jedoch nicht modelliert.

Cheong et al. [19]: Cheong et al. beschreiben mit ihrem Modell das Wachstum einer festen Hülle in einem trocknenden Suspensionstropfen, wobei die Struktur der Hülle nicht beschrieben wird. Das Modell konzentriert sich auf die Beschreibung der Trocknungsvorgänge, nachdem sich eine feste Hülle um den Tropfen gebildet hat, bis er komplett getrocknet ist. Die Temperatur im Tropfen wird als konstant über dem Radius angenommen.

Nešić und Vodnik [20]: Zunächst wird die Verdunstung und damit die Massenabnahme eines Lösungstropfens mit einer freien Flüssigkeitsoberfläche berechnet. Aus der sich ändernden Masse des Tropfens ergibt sich der aktuelle Radius. Die Tropfentemperatur sowie die radiale Konzentrationsverteilung der gelösten Substanz im Tropfen werden berechnet. Dabei wird der Einfluß der Konzentration der gelösten Substanz auf den Dampfdruck am Tropfenrand berücksichtigt. Die radiale Temperaturverteilung im Tropfen wird als konstant angenommen. Die Dicke der sich im Laufe des Trocknungsprozesses ausbildenden Tropfenhülle wird aus einer Massenbilanz ermittelt und in den Gleichungen für den Stoff- und Wärmeübergang berücksichtigt. Eine detaillierte Modellierung der Struktur der Hülle wird nicht durchgeführt.

Lee und Law [21]: Lee und Law haben ein Modell entwickelt, das den Trocknungsprozeß in einem Tropfen, der aus Treibstoff mit darin suspendierten Feststoffpartikeln besteht, beschreibt. Zunächst wird die Bildung einer festen Schale mit Hilfe einer Massenbilanz beschrieben, wobei die Porosität der Hülle berücksichtigt wird. Das Modell berücksichtigt außerdem die Bildung einer Gasblase im Inneren, wobei jedoch keine Drücke berechnet werden, so daß ein späteres Aufblähen der Hülle nicht simuliert werden kann.

Bhatia und Sirignano [22]: Bhatia und Sirignano beschäftigen sich ebenfalls mit dem Trocknungsprozeß bei der Verbrennung. Das Modell beschreibt wie das von Lee und Law die Verdunstung eines Treibstofftropfens mit suspendierten Partikeln. Die Entwicklung der Hüllendicke wird ebenfalls aus einer Massenbilanz berechnet. Im Inneren des Tropfens wird ebenfalls die Bildung einer Gasblase berücksichtigt, wobei angenommen wird, daß die Gasblase aus Trocknungsgas und verdampftem Treibstoff besteht.

Elperin und Krasovitov [23]: Das Modell beschreibt die Trocknungsvorgänge in einem Suspensionstropfen. Im Tropfen werden die radiale Konzentrationsverteilung der Suspensionspartikel und die Temperatur berechnet, wobei die Temperatur über dem Radius als konstant angenommen wird. Sobald sich eine feste Hülle ausgebildet hat werden die Stoff- und Wärmetransportwiderstände der Schale berücksichtigt. Die Partikel werden über Volumenanteile 
berücksichtigt.

Ford [24]: Als Feststoffbildungsprozeß wird in dem Einzeltropfenmodell die Kristallisation berücksichtigt. Es wird die zeitliche Änderung der Konzentrationsverteilung im Tropfen und die damit verbundene Kristallisation simuliert, wobei der Stofftransport im Tropfen durch Diffusion stattfindet. Zur Berechnung der Partikelgrößenverteilung werden Populationsbilanzen verwendet. Die Dicke der festen Hülle wird aus einer Massenbilanz berechnet. Allerdings wird die Struktur der sich bildenden Hülle nicht mit Hilfe der Partikelgrößenverteilungen beschrieben. Sobald sich eine feste Hülle ausgebildet hat, wird die radialsymmetrische Berechnung beendet und die Dicke der Hülle aus der noch gelösten Salzmenge berechnet, wobei als Porosität in der Schale die der dichtesten Kugelpackung angenommen wird.

Hecht und King [25]: Bei dem Modell von Hecht und King handelt es sich um eine Weiterentwicklung des Modells von Sano und Keey [18] für eine Zuckerlösung. In dem Modell wird der Stofftransport in einem Dreikomponentensystem bestehend aus Wasser, Zucker und Schwefelhexafluorid $\left(S F_{6}\right)$ modelliert. Die Stofftransportgleichung des Mehrkomponentengemisches ist in der Form des Fickschen Gesetzes aufgestellt. Im Inneren wird die Bildung einer Blase simuliert, die zu einem Aufblähen, jedoch nicht zum Platzen des Partikels führt.

Liang et al. [26]: Liang et al. haben ein Modell entwickelt, das die Vorgänge in einem trocknenden Suspensionstropfen beschreibt, bis sich eine feste Hülle ausgebildet hat. Zur Modellierung der Transportvorgänge im Tropfen werden Gleichungen aus der Schüttgutmechanik verwendet, wobei die Suspensionspartikel als Volumenanteil berücksichtigt werden. Aus einer Bilanz um ein Volumenelement ergibt sich eine Transportgleichung für die Flüssigkeit, die ähnlich der Diffusionsgleichung ist. Die Triebkraft für den Flüssigkeits- und Partikeltransport ergibt sich aus einer Energiebetrachtung an der Tropfenoberfläche. Der Partikeltransport erfolgt im Tropfen in entgegengesetzter Richtung zum Flüssigkeitstransport und ist von gleicher Größe.

Brenn [27]: In seinem Modell hat Brenn eine dimensionslose Kennzahl G entwickelt, deren Größe darüber entscheidet, ob ein Voll- oder Hohlpartikel entsteht. Der Stofftransport im Tropfen wird mit äquimolarer Diffusion berechnet. Die Kennzahl G ergibt sich aus dem Verhältnis der Diffusionskoeffizienten der gelösten Substanz im Tropfen und dem Diffusionskoeffizienten im Trocknungsgas sowie dem Verhältnis der Dichten. Der Trocknungsprozeß wird nicht wie in der Trocknungstechnik üblich in verschiedenen Trocknungsabschnitten getrennt beschrieben. In einem weiteren Modell hat er die zeitliche Änderung der Konzentrationsverteilung der gelösten Substanz in einem trocknenden Tropfen berechnet [30], wobei die Diffusionsgleichung analytisch gelöst wird.

Weiß [28]: Das eindimensionale, radialsymmetrische Modell simuliert die Kristallisation in einem verdampfenden Lösungstropfen. Im Tropfen wird die zeitliche Änderung der Konzen- 
trationsverteilung über dem Radius berechnet, wobei in der Salzlösung äquimolare Diffusion angenommen wird. In Abhängigkeit von der sich ausbildenden Übersättigung kommt es im schrumpfenden Tropfen zur Kristallisation. Zur Beschreibung der Partikelverteilung im Tropfen werden Populationsbilanzen eingesetzt. Auf diese Weise kann die Anzahldichteverteilung der Partikel über dem Radius und der Partikelgröße berechnet werden. Dabei bewegen sich die im Tropfen entstehenden Partikel mit dem schrumpfenden Koordinatensystem nach innen. Zur Beschreibung der Entstehung und des Wachstums der Partikel werden Kristallisationskinetiken aus der Literatur verwendet. Im Tropfen wird eine gleichmäßige Temperaturverteilung angenommen. Das Hüllenwachstum wird durch das Modell nicht beschrieben.

Kadja und Bergeles [29]: Das eindimensionale, radialsymmetrische Modell beschreibt sowohl die Bewegung eines Suspensionstropfens im Gas als auch die Verdampfung des Lösungsmittels und die Temperaturentwicklung im Tropfen. Dabei wird eine über dem Tropfenradius konstante Temperatur angenommen. Die radiale Verteilung der Suspensionspartikel wird nicht modelliert. Sobald sich eine feste Hülle ausgebildet hat, werden die zusätzlichen Widerstände der festen Hülle für den Stoff- und Wärmetransport berücksichtigt. Die Dicke der sich ausbildenden festen Hülle wird analytisch aus dem Feststoffmassenanteil im Tropfen berechnet, wobei ein über dem Tropfenradius konstanter Feststoffvolumenanteil angenommen wird. Die Struktur der festen Hülle wird nicht detailliert betrachtet.

Neben diesen Modellen werden auch weniger komplexe Modelle verwendet, um das Trocknungsverhalten von Lösungs- oder Suspensionstropfen zu beschreiben. Dazu gehören das CDRC- (characteristic drying rate curve) und REA-Modell (reaction engineering approach), die von Chen und Lin [31] mit experimentellen Untersuchungen zur Trocknung von entrahmter Milch verglichen worden sind. Keines der Modelle berücksichtigt die Verteilung der Feuchtigkeit im Tropfen. Das CDRC-Modell geht von der Annahme aus, daß der Trocknungsverlauf aus dem Verhältnis aktuelle Verdunstungsrate zu Verdunstungsrate bei ungehinderter Verdunstung berechnet werden kann. Die Verdunstungsrate des Tropfen berechnet sich aus dem freien Flüssigkeitsgehalt und dem Wärmeübergang auf den Tropfen. Beim REA-Modell wird angenommen, daß die Verdunstung ein Aktivierungsprozeß ist. In der Beziehung, welche die zeitliche Änderung der Tropfenmasse beschreibt, wird daher eine Aktivierungsenergie berücksichtigt. Diese Aktivierungsenergie ist gleich Null, wenn der Tropfen eine flüssige Oberfläche hat. 


\subsection{Berechnung von Verteilungsgrößen mit Populationsbilan- zen}

Zur Berechnung von Verteilungsgrößen werden Populationsbilanzen eingesetzt. Insbesondere in der Partikeltechnik werden Populationsbilanzen zur Simulation von Prozessen wie Kristallisation, Fällung, Granulation, Polymerisation, Zerkleinerung oder Verbrennung verwendet [32]. Mit Populationsbilanzen werden u.a. die Größenverteilung und der Agglomerationsgrad der Partikel beschrieben. In der Regel wird mit Populationsbilanzen nur eine Partikeleigenschaft beschrieben, weil die Berücksichtigung einer weiteren Dimension mathematisch aufwendig ist und auch viel Rechenkapazität erfordert. Zur Untersuchung von räumlichen Verteilungen werden sie auch mit CFD-Software gekoppelt [33]. Ein Beispiel für den Einsatz zur Berechnung räumlicher Verteilungen ist die Simulation von Blasensäulen [34]. Ford [24] und Weiß [28] haben Populationsbilanzen zur Beschreibung der Partikelverteilung im schrumpfenden Tropfen verwendet. Dabei werden der radiale Ort und die Partikelgröße jedes Partikels bilanziert. In dem Modell von Weiß bewegen sich die Partikel jedoch an jedem radialen Punkt des Tropfens mit dem Gitter mit, so daß der radiale Ort nicht mehr durch die Populationsbilanz erfaßt wird. Auch Ford hat sich nicht die Möglichkeit zunutze gemacht, die radiale Lage der Partikel durch die Populationsbilanzen zu beschreiben. Weitere Arbeiten, in denen Populationsbilanzen mit normierten beweglichen Koordinatensystemen eingesetzt werden, sind nicht bekannt.

\subsection{Problemstellung und Zielsetzung}

Die Grobauslegung von Sprühtrocknern erfolgt zum heutigen Stand der Technik zunächst auf Basis von Energie- und Massenbilanzen um den gesamten Prozeß. Für die Detailauslegung wird dann auf Erfahrungen aus bisher bestehenden Prozessen zurückgegriffen. Zur Optimierung der Form des Trocknungsraumes und der Zuführung des Trocknungsgases steht leistungsfähige Simulationssoftware aus dem Bereich der Strömungssimulation zur Verfügung. Für ein besseres Verständnis der mikroskopischen Vorgänge während der Trocknung ist in den letzten Jahren eine Reihe von Modellen entwickelt worden, die die mikroskopischen Feststoffbildungsprozesse in Einzeltropfen beschreiben. Allen Modellen ist jedoch gemeinsam, daß sie eine Reihe von Vereinfachungen vornehmen. Um die Endstruktur des Produktes vorhersagen zu können, ist jedoch eine detailliertere Modellierung der mikroskopischen Prozesse notwendig. Daher wird in dieser Arbeit ein Modell entwickelt, welches die mikroskopische Struktur des Produkts modelliert. Die Modellierung der Verteilung der Feststoffpartikel im Tropfen erfolgt dabei mit Hilfe von Populationsbilanzen. Im Gegensatz zu bisherigen Arbeiten wird dabei von den Möglichkeiten der Beschreibung des Ortes der Partikel mit Populationsbilanzen in einem normierten, beweglichen Koordinatensystem Gebrauch gemacht werden. 


\section{Kapitel 3}

\section{Physikalische Grundlagen der Tropfentrocknung}

\subsection{Grundlagen der Trocknung}

\subsubsection{Wärme- und Stoffübergang}

Der Wärmeübergang an überströmten Einzelkörpern wird mit Hilfe der dimensionslosen NusseltZahl dargestellt, die aus dem Wärmeübergangskoeffizienten $\alpha$ im Gas, einer Charakteristischen Überströmlänge $l$ und der Wärmeleitfähigkeit $\lambda$ des Gases gebildet wird.

$$
N u=\frac{\alpha l}{\lambda}
$$

Die Nusselt-Zahl ist eine Funktion der Reynolds-Zahl

$$
R e=\frac{v l}{\nu},
$$

welche sich aus der Geschwindigkeit des Gases $v$, der Überströmlänge $l$ und der kinematischen Viskosität $\nu$ zusammensetzt und die Ausbildung einer hydrodynamischen Grenzschicht charakterisiert, sowie der Prandtl-Zahl

$$
\operatorname{Pr}=\frac{\nu}{a} \operatorname{mit} a=\frac{\alpha l}{\lambda},
$$

welche sich aus der kinematischen Viskosität $\nu$, dem Wärmeübergangskoeffizienten $\alpha$ im Gas, einer Charakteristischen Überströmlänge $l$ und der Wärmeleitfähigkeit des Gases zusammensetzt und dem Verhältnis der thermischen zur hydrodynamischen Grenzschichtdicke entspricht. Bei Gasen ist die Strömungsgrenzschicht geringfügig dünner als die Temperaturgrenzschicht $(0,6 \leq \operatorname{Pr} \leq 1)$ [35].

$$
N u=f(R e, P r)
$$


Die Nusselt-Zahl wird mit Hilfe empirischer Gleichungen für verschiedene Körper in Abhängigkeit vom Strömungszustand und der Form des überströmten Körpers berechnet.

Der Stoffübergang an überströmten Einzelkörpern wird mit der Sherwood-Zahl

$$
S h=\frac{\beta l}{D}
$$

beschrieben, wobei $\beta$ der Stoffübergangskoeffizient ist. Analog zur Nusselt-Zahl ist die SherwoodZahl eine Funktion der Reynolds-Zahl. Im Gegensatz zur Nusselt-Zahl ist die Sherwood-Zahl jedoch nicht von der Prandtl-Zahl, sondern von der Schmidt-Zahl

$$
S c=\frac{\nu}{D}
$$

abhängig, die sich aus der kinematischen Viskosität $\nu$ und dem Diffusionskoeffizienten $D$ berechnet. Die Sherwood-Zahl wird ebenfalls als Funktion dimensionsloser Kennzahlen dargestellt.

$$
S h=f(R e, S c)
$$

Für kleine Reynolds-Zahlen streben die Nusselt- und Sherwood-Zahl einem unteren Grenzwert zu. Für Kugeln beträgt dieser Wert 2. Ist eine Kugel von einem ruhenden Fluid umgeben, so wird die Wärme ausschließlich durch Leitung übertragen, und aus einer Energiebilanz

$$
2 \lambda d \pi\left(\vartheta_{O}-\vartheta_{U}\right)=\alpha \pi d^{2}\left(\vartheta_{O}-\vartheta_{U}\right)
$$

ergibt sich $\mathrm{Nu}=2[35,36]$, wobei $\vartheta_{O}$ die Wandtemperatur und $\vartheta_{U}$ die Umgebungstemperatur bezeichnet. Nach [35] ist der Wärmeübergang bei einem quer angeströmten kreisförmigen Körper sowohl am Staupunkt als auch im Bereich der Strömungsablösung am größten. Wegen der Analogie von Stoff- und Wärmeübergang sind die gleichen Verhältnisse für den Stoffübergang anzunehmen.

\subsubsection{Verdunstung/Verdampfung}

Bei der Verdunstung diffundiert der Dampf aufgrund eines Partialdruckgefälles von der Flüssigkeitsoberfläche in das Gas, während das Gas nicht in die Flüssigkeit diffundieren kann. Wegen des Partialdruckgefälles im Gas muß Gas jedoch immer entgegen dem Dampf diffundieren. Zur Kompensation des Diffusionsstroms des Gases überlagert sich daher ein Ausgleichsstrom des Dampfes (Abb. 3.1).

$$
\dot{m}_{\text {Dampf }}=-A \frac{D}{R_{\text {Dampf }} T} \frac{d p_{\text {Dampf }}}{d l}+\dot{m}_{\text {Dampf }} \frac{p_{\text {Dampf }}}{p}
$$

Daraus ergibt sich durch Umformung

$$
\dot{m}_{\text {Dampf }}=A \frac{D}{R_{\text {Dampf }} T} p \frac{d \ln \left(p-p_{\text {Dampf,U }}\right)}{d l} .
$$




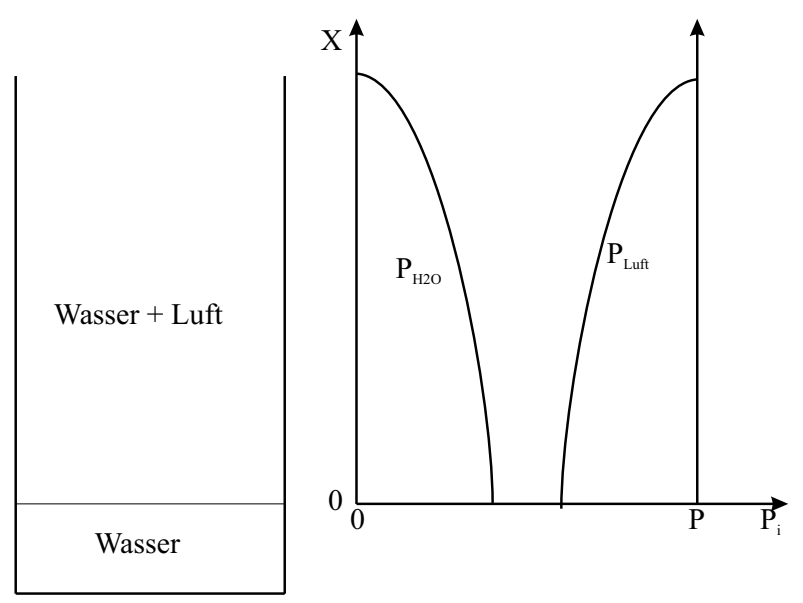

Abbildung 3.1: Links: Luftsäule über einem Wasserspiegel, rechts: Verlauf der Partialdrücke von Wasserdampf $p_{\mathrm{H}_{2} \mathrm{O}}$ und Luft $p_{\text {Luft }}$ bei der Verdunstung über dem Wasserspiegel [35]

Sind die Partialdrücke klein gegenüber dem Gesamtdruck, so vereinfacht sich die Gleichung $\mathrm{zu}$

$$
\dot{m}_{\text {Dampf }}=A \frac{D}{R_{\text {Dampf }} T} \frac{1}{l}\left(p_{\text {Dampf,O}}-p_{\text {Dampf,U }}\right) .
$$

$R_{\text {Dampf }}$ bezeichnet dabei die spezielle Gaskonstante [36]. Erreicht die Temperatur $T$ den Siedepunkt, so wird der Dampfpartialdruck $p_{\text {Damp }, O}$ gleich dem Gesamtdruck $p$ und somit der Dampfmassenstrom $\dot{m}_{\text {Dampf }}$ unendlich. Der Stofftransport findet dann aufgrund absoluter Druckunterschiede statt, welche für die Überwindung äußerer Widerstände verbraucht werden [36].

$$
\dot{m}_{\text {Dampf }}=\frac{1}{h_{v}} \frac{1}{\frac{1}{\alpha}+\frac{s}{\lambda}}\left(\vartheta_{L}-\vartheta_{O}\right)
$$

Die treibende Kraft für den Stofftransport ist also die Temperaturdifferenz zwischen der Oberfläche und dem Gas. Bei Temperaturen nahe dem Siedepunkt bestimmen sowohl der Wärmetransport als auch die Diffusion den Massentransport.

\subsubsection{Sattdampfdruck}

Der Sattdampfdruck an der Flüssigkeitsoberfläche läßst sich mit Hilfe der Antoine-Gleichung

$$
p_{\text {Dampf }, O}=10^{A-\frac{B}{T+C-273,15}}
$$

berechnen. Für Wasser sind die Konstanten $\mathrm{A}=5,11564, \mathrm{~B}=1687,537$ und $\mathrm{C}=230,17$ [37].

\subsubsection{Kühlgrenztemperatur}

Strömt warme Luft entlang eines feuchten Körpers, so nimmt die Feuchte der Luft zu und die Temperatur nimmt wegen der Wärmeabgabe an das Gut ab. Die Kühlgrenztemperatur ist die 


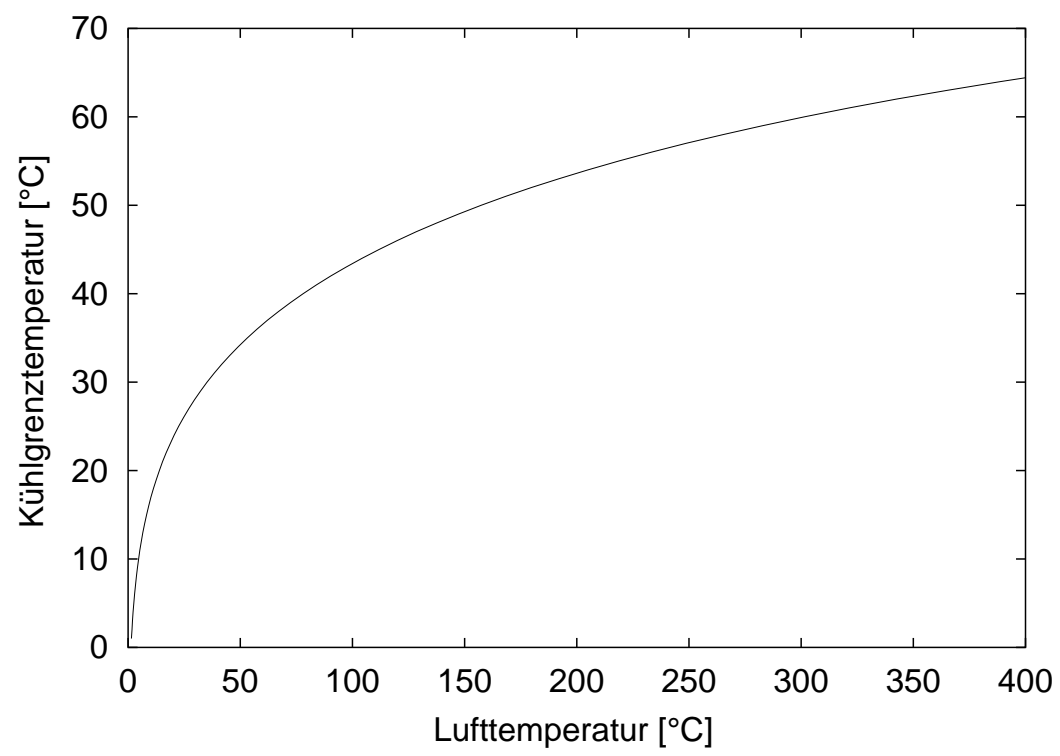

Abbildung 3.2: Kühlgrenztemperatur bei der Verdunstung von Wasser in Luft $\left(p_{u}=0\right)$ in Abhängigkeit von der Lufttemperatur nach [36]

Temperatur, welche die Trocknungsluft annimmt, wenn sich die Luft vom Eintrittszustand bis zur vollständigen Sättigung mit Feuchte anreichert. Sie hängt ausschließlich vom Anfangszustand des Trocknungsgases ab (Abb. 3.2) [36]. Bereits nach einer kurzen Zeit nimmt das Trocknungsgut ebenfalls die Kühlgrenztemperatur an.

$$
\begin{gathered}
\vartheta_{\text {Luft }}-\vartheta_{\text {Oberfläche }}=\frac{h_{v}\left(\theta_{0}\right)}{c_{p D}}\left[(1+B)^{\gamma}-1\right], \\
\text { mit } B=\frac{p_{\text {Dampf }, O}-p_{\text {Dampf }, U}}{p-p_{\text {Dampf }, O}} \\
\text { und } \gamma=\frac{c_{p \text { Dampf }} M_{\text {Dampf }}}{\overline{c_{p} M}} \cdot\left(\frac{a}{\delta}\right)^{-(1-n)}
\end{gathered}
$$

Hierin ist $p_{\text {Dampf,O }}$ der Dampfpartialdruck an der Oberfläche, $p_{\text {Dampf,U }}$ der Dampfpartialdruck in der Umgebung, p der Gesamtdruck, $c p_{\text {Dampf }}$ die Wärmekapazität des Dampfes, $M_{\text {Dampf }}$ die molare Masse des Dampfes, $\delta$ der Diffusionskoeffizient, $a$ der Temperaturleitkoeffizient und $B$ das Dampfdruckverhältnis. n wird für den Fall vollkommener Turbulenz oder Pr $=$ Sc zu 1 und bei ruhender Luft zu 0. Der Wert von $\gamma$ liegt im Bereich von 1 (für Wasserdampf-Luft gilt $\gamma \approx 1,3)[36]$. 


\subsubsection{Trocknung von Tropfen}

Bei einem verdunstenden Tropfen nimmt der quadrierte Durchmesser linear mit der Zeit ab. Das sogenannte $d^{2}$-Gesetz ergibt sich aus der Integration der Gleichung

$$
-(d / 2) \frac{d(d / 2)}{d t}=\frac{S h D}{2} \frac{\left(p_{o}-p_{u}\right)}{R_{D} T \rho}
$$

wobei sich dann ein linearer Zusammenhang zwischen der Abnahme des quadrierten Radius und der Zeit ergibt [8]:

$$
\frac{1}{2}\left((d / 2)_{0}^{2}-(d / 2)^{2}\right)=\frac{S h D}{2} \frac{\left(p_{o}-p_{u}\right)}{R_{D} T} t
$$

Bei $S h=2$ erhält man

$$
(d / 2)_{0}^{2}-(d / 2)^{2}=2 D \frac{\left(p_{o}-p_{u}\right)}{R_{D} T} t
$$

\subsubsection{Genereller Trocknungsverlauf}

Der trocknende Tropfen kann als poröser Körper betrachtet werden, sobald sich größere Mengen an Feststoffpartikeln im Tropfen gebildet haben. Im Unterschied zu den in der Trocknungstechnik beschriebenen Körpern ändert sich bei der Sprühtrocknung der Feststoffgehalt im Tropfen. Bei porösen Körpern stellt sich zunächst überall eine gleichmäßige konstante Temperatur ein, solange so viel Flüssigkeit durch Kapillarkräfte an die Körperoberfläche transportiert werden kann, wie an dieser verdunstet. Dieser Abschnitt konstanter Trocknungsgeschwindigkeit wird als 1 . Trocknungsabschnitt bezeichnet. Die Verdunstung ist nur von den Widerständen in der umgebenden Gasphase abhängig, weil die Verdunstung an der Körperoberfläche stattfindet. Während des 1 . Trocknungsabschnitts ändert sich nur die Flüssigkeitsverteilung im Körper. Wenn nicht mehr genügend Flüssigkeit an die Oberfläche transportiert werden kann, bewegt sich der Trocknungsspiegel ins Innere des Trocknungsguts. Die Verdunstung findet nun im Inneren des Körpers statt, und der Dampf muß an die Oberfläche des Körpers diffundieren. Der Dampfmassenstrom wird also geringer, und somit verlangsamt sich der Trockungsprozeß. Die zur Verdunstung notwendige Wärme muß hauptsächlich durch Leitung an den Trocknungsspiegel transportiert werden. Dieser Abschnitt wird als 2. Trocknungsabschnitt bezeichnet. Bei hygroskopischen Stoffen beginnt der 2. Abschnitt, wenn der maximale hygroskopische Feuchtigkeitsgehalt an der Oberfläche unterschritten wird. Wenn im gesamten Körper der maximale hygroskopische Feuchtigkeitsgehalt erreicht wird, beginnt ein 3. Trocknungsabschnitt, bei dem die Trocknungsgeschwindigkeit dem Wert 0 zustrebt, wenn die Gleichgewichtsfeuchte erreicht ist [36]. Bei nichtporigen Körpern, in denen keine Dampfdiffusion möglich ist (z.B. Seife), findet die Trocknung ausschließlich an der Oberfläche statt, und der Trocknungsprozeß läßst sich nicht in Abschnitte unterteilen [36]. 


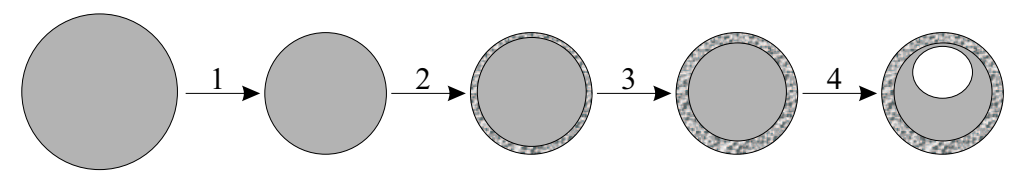

Abbildung 3.3: Physikalisches Modell des Trocknungsprozesses (Beschreibung siehe Kapitel $3.2)$

\subsection{Trocknungsverlauf bei Lösungstropfen}

Die Vorgänge bei der Trocknung eines Lösungs- bzw. Suspensionstropfens werden seit etwa 50 Jahren experimentell untersucht [38]. Zur Untersuchung werden Tropfen entweder am Draht oder einer Scheibe in einem Heißgasstrom aufgehängt, oder es werden Tropfenketten in einem Fallturm getrocknet und aufgefangen. Auf Basis der Beobachtungen läßt sich der Trocknungsverlauf beschreiben [39, 38]. Im ersten Trocknungsabschnitt hat der Tropfen eine flüssige Oberfläche und nimmt die Kühlgrenztemperatur an (Schritt 1, Abb. 3.3). Die Wärmeund Stofftransportwiderstände liegen ausschließlich in der Gasphase und werden aus Nusseltund Sherwood-Zahl berechnet. Wegen der Verdunstung des Lösungsmittels konzentriert sich die gelöste Substanz vor allen Dingen am Tropfenrand auf. Sobald die Sättigungskonzentration überschritten ist, beginnt der Kristallisationsprozeß (Schritt 2, Abb. 3.3). Aufgrund des radialen Konzentrationsverlaufs und der Bewegung der Phasengrenze, die die Partikel am Tropfenrand mit nach innen bewegt, konzentrieren sich die Partikel am Tropfenrand. Wenn die Zahl der Partikel zu gering ist oder der Trocknungsprozeß sehr langsam ist, kann sich keine Hülle ausbilden. Es entstehen dann bevorzugt Vollpartikel, weil bei langsamer Verdunstung auch im Tropfeninneren Partikel aufgrund des Konzentrationsausgleichs der gelösten Substanz entstehen können. Sobald eine feste Hülle vorhanden ist, beginnt der 2. Trocknungsabschnitt (Schritt 3, Abb. 3.3). Zu den Wärme- und Stofftransportwiderständen in der Gasphase kommen nun die Widerstände der festen Hülle hinzu. Die Temperatur im Tropfen kann aufgrund des zusätzlichen Stofftransportwiderstands in der Hülle die Kühlgrenztemperatur übersteigen. Im Inneren des Tropfens kann sich schließlich eine aus Trocknungsgas oder Lösungsmitteldampf bestehende Blase bilden (Schritt 4, Abb. 3.3). Wird die Flüssigkeit durch Kapillarkräfte an die Oberfläche gesogen, so fällt der Druck im Tropfeninneren ab, und Lösungsmittel kann verdampfen. Durch das Eindringen von Trocknungsgas durch trocken gefallene Kapillaren kann es jedoch auch wieder zu einem Druckausgleich kommen. Im Falle einer undurchlässigen Hülle verdampft das Lösungsmittel im Inneren, und das Partikel bläht sich auf und kollabiert am Ende des Trocknungsprozesses infolge der Kondensation des Lösungsmittels. Übersteigen die Druckkräfte im Tropfen die Festigkeit der Hülle, so kommt es zum Bruch. Am Ende des Prozesses steht entweder ein Hohlkörper mit einer festen porösen Hülle, ein Vollkörper oder 


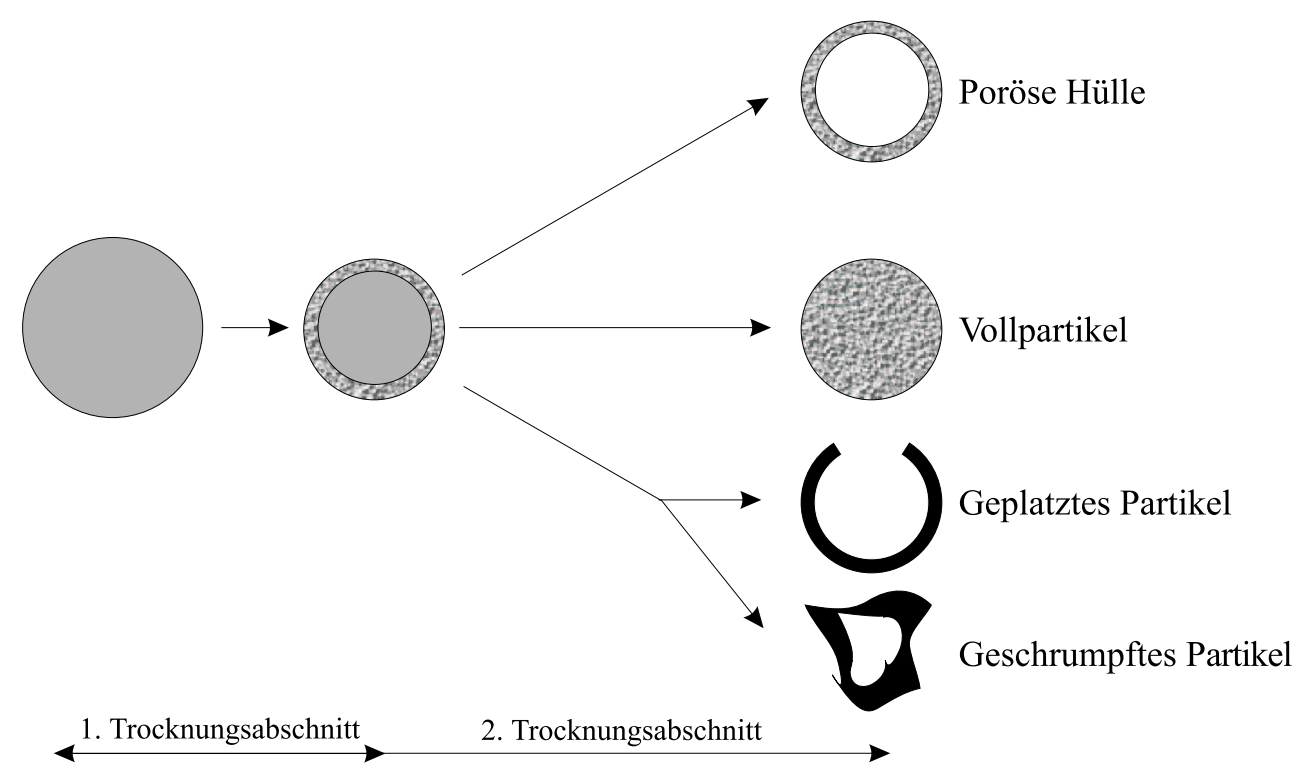

Abbildung 3.4: Morpholgien sprühgetrockneter Partikel

ein Hohlkörper mit einer festen undurchlässigen und ggf. geplatzten Hülle. Abb. 3.4 zeigt verschiedene mögliche Morphologien. Undurchlässige Hüllen treten bei filmbildenden Substanzen auf, wohingegen poröse Hüllen bei Suspensionen und kristallisierenden Substanzen auftreten.

\subsection{Stabilität des Tropfens und Strömungen im Tropfen}

Aufgrund der Oberflächenspannung der Lösung nimmt ein frei fallender Tropfen die Kugelform an, weil so die Oberflächenenergie am geringsten ist. Bewegt sich ein Tropfen relativ zu einem umgebenden Medium, so wirken auf den Tropfen Druck- und Reibungskräfte. Diese bewirken das Auftreten von Strömungen im Tropfen sowie seine Verformung [5]. Die auf den Tropfen wirkenden Kräfte sind abhängig von der Relativgeschwindigkeit des Tropfens im Gas. Die Weber-Zahl

$$
W e=\frac{\rho_{a} v^{2} d}{\sigma}
$$

stellt das Verhältnis von aerodynamischem Widerstand zur Oberflächenspannung dar, wobei $\rho_{a}$ die Viskosität des Gases, $v$ die Geschwindigkeit, $d$ der Tropfendurchmesser und $\sigma$ die Oberflächenspannung ist. Solange $W e / 8 \lll 1$ ist, wird die Kugelform des Tropfens durch die Oberflächenspannung gewährleistet. Wesentliche Abweichungen von der Kugelgestalt treten erst ab Weber-Zahlen $>6$ auf, was einem Wassertropfen in Luft mit einem Durchmesser von $4 \mathrm{~mm}$ entspricht [40]. Durch die tangentiale Schubspannung des Gases an der Tropfenoberfläche bilden sich im Tropfen Wirbel, was wiederum zu einer Erhöhung der Fallgeschwindigkeit aufgrund der Abnahme der Reibung führt. Die Schubspannung an der Tropfenoberfläche be- 


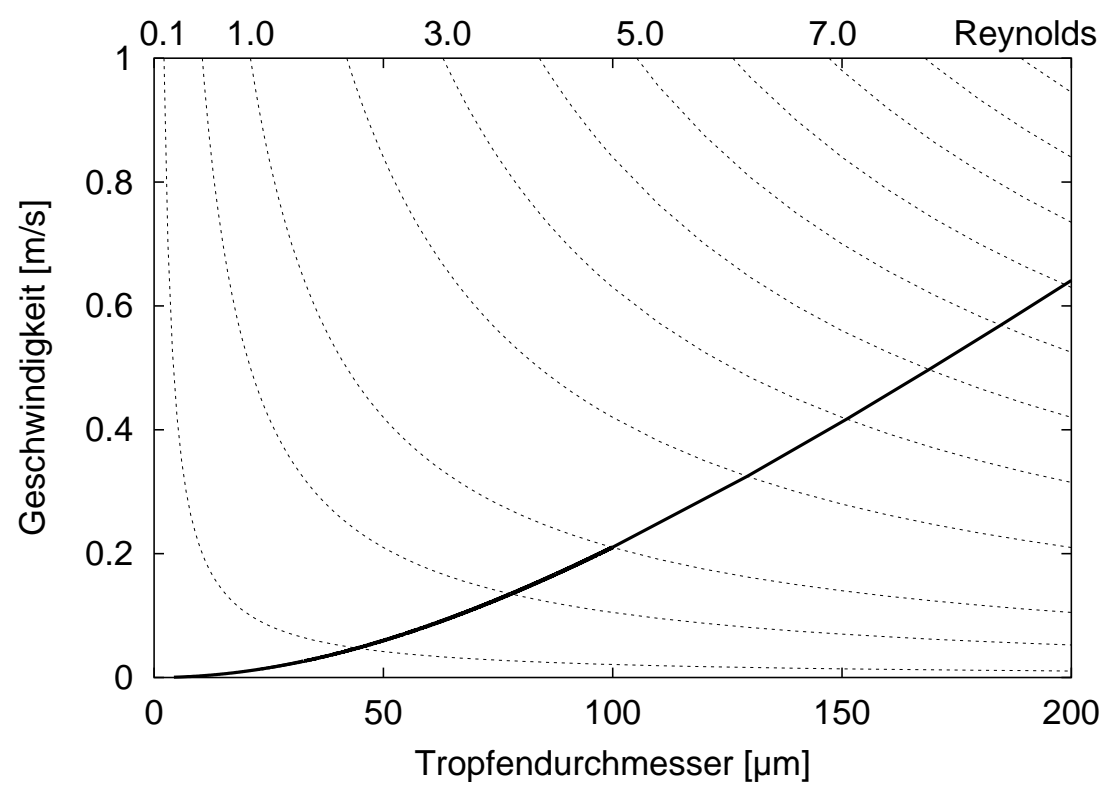

Abbildung 3.5: Fallgeschwindigkeit und Reynolds-Zahl eines Tropfens in ruhender Luft in Abhängigkeit vom Durchmesser

rechnet sich nach [12] zu

$$
\tau=\eta r \frac{\partial}{\partial r}\left(\frac{v_{\theta}}{r}\right)+\frac{\eta}{r} \frac{\partial v_{r}}{\partial \theta}
$$

wobei $\eta$ die dynamische Viskosität, $v_{\theta}$ die Geschwindigkeitskomponente mit Winkel $\theta$ und $v_{r}$ die radiale Richtungskomponenten der Geschwindigkeit ist. Im Sprühtrockner ist die Relativgeschwindigkeit jedoch gering, weil die Tröpfchen in der Regel einen Durchmesser von nur wenigen $100 \mu \mathrm{m}$ haben. Abbildung 3.5 zeigt, daß ein in ruhender Luft frei fallender Wassertropfen mit einem Durchmesser von $200 \mu$ m nur eine Reynolds-Zahl von ungefähr 6 erreicht. Diese Geschwindigkeit wird in sehr kurzer Zeit erreicht, das heißt, die Phase der Beschleunigung ist sehr kurz. Darüber hinaus ist die Viskosität des Lösungstropfens sehr viel größer als die des umgebenden Trocknungsgases. Nach [40] entspricht die Fallgeschwindigkeit eines Tropfens einer hochviskosen Flüssigkeit in einem Gas der einer Feststoffkugel der gleichen Größe und Masse. Aus der Gleichung für den Widerstand eines kugelförmigen Tropfens nach Ribtschinski und Hadamard [40]

$$
W=\pi d v \eta_{a}\left(2 \eta_{a}+3 \eta_{i}\right) /\left(\eta_{a}+\eta_{i}\right)
$$

ergibt sich wieder die Stokessche Formel, wenn die Viskosität $\eta_{i}$ im Tropfen sehr viel höher ist als in der Gasphase, dabei sind $\eta_{a}$ und $\eta_{i}$ die Viskosität der Gasphase bzw. des Tropfens, $v$ die Geschwindigkeit und $d$ der Durchmesser. Aus dem Kräftegleichgewicht am Tropfen berechnet 
sich die Fallgeschwindigkeit zu

$$
v=\frac{g\left(\rho_{i}-\rho_{a}\right) d^{2}\left(\eta_{a}-\eta_{i}\right)}{6 \eta_{a}\left(2 \eta_{a}+3 \eta_{i}\right)} .
$$

Wenn $\rho_{a} / \rho_{i} \rightarrow \infty$ und $\eta_{a} / \eta_{i} \rightarrow \infty$, dann entsteht wieder die Stokessche Gleichung. Daher kann davon ausgegangen werden, daß im Tropfen keine Strömungen auftreten.

Zusammenfassend kann man sagen, daß in den kleinen Tröpfchen bei der Sprühtrocknung keine Zirkulationen auftreten, weil sie sehr schnell ihre Endgeschwindigkeit erreicht haben, aufgrund ihrer geringen Größe nur eine sehr geringe Relativgeschwindigkeit zum Gas erreichen und weil die Viskosität der Flüssigkeit sehr viel größer ist als die des Gases.

\subsection{Hüllenwiderstände}

Die feste Hülle bildet im zweiten Trocknungsabschnitt einen Widerstand sowohl für den Wärme- als auch den Stofftransport.

\subsubsection{Wärmetransportwiderstand}

Die Wärme aus dem Trocknungsgas wird zunächst auf die feste Oberfläche übertragen und durch die Hülle zur Flüssigkeit weitergeleitet. Im Falle einer porösen Hülle berühren sich die Partikel jedoch nur an bestimmten Stellen, so daß die Wärmeleitung schlechter ist als in einem Festkörper. Es gibt mehrere Möglichkeiten, die Wärmeleitfähigkeit in porösen Körpern zu berechnen. Für den Grenzfall guter Wärmeleitung $\lambda$, wenn die Partikel durch zahlreiche Feststoffbrücken verbunden sind, wird diese mit

$$
\lambda=\epsilon \lambda_{\text {Partikel }}+(1-\epsilon) \lambda_{\text {Luft }}
$$

berechnet, wobei $\epsilon$ der Feststoffvolumenanteil ist [36]. Die Feststoffschichten liegen parallel in Richtung des Wärmestroms getrennt durch Luftschichten, wodurch die Wärmeleitung nicht so stark behindert wird. Im Fall schlechter Wärmeleitung, wenn die Partikel nur durch wenige Feststoffbrücken verbunden sind, läßt sie sich mit

$$
\lambda=\frac{1}{\frac{\epsilon}{\lambda_{\text {Partikel }}}+\frac{(1-\epsilon)}{\lambda_{\text {Luft }}}}
$$

berechnen [36]. In diesem Fall liegen die Feststoffschichten senkrecht zur Richtung des Wärmestroms und sind durch Luftschichten getrennt, wodurch die Wärmeleitung stark behindert wird. Darüber hinaus kann die Wärmeleitfähigkeit der Hülle $\lambda_{\text {Hülle }}$ auch wie die einer Schüt- 
tung berechnet werden.

$$
\begin{gathered}
\frac{\lambda_{\text {Hülle }}}{\lambda_{\text {Luft }}}=1-\sqrt{1-\epsilon}+\sqrt{1-\epsilon} \cdot \frac{2}{1-\frac{\lambda_{\text {Partikel }}}{\lambda_{\text {Luft }}}} \cdot \\
\left(\frac{1-\frac{\lambda_{\text {Partikel }}}{\lambda_{\text {Luft }}} B}{\left(1-\frac{\lambda_{\text {Partikel }}}{\lambda_{\text {Luft }}} B\right)^{2}} \cdot \ln \frac{\lambda_{\text {Partikel }}}{B \lambda_{\text {Luft }}}-\frac{B+1}{2}-\frac{B-1}{1-\frac{\lambda_{\text {Partikel }}}{\lambda_{\text {Luft }}}}\right)
\end{gathered}
$$

B ist ein experimentell ermittelter Verformungsparameter und wird aus

$$
B=C\left(\frac{\epsilon}{1-\epsilon}\right)^{10 / 9}
$$

berechnet. Für Kugeln ist $\mathrm{C}=1,25[36]$.

\subsubsection{Stofftransportwiderstand}

Der Lösungsmitteldampf, der an der Flüssigkeitsoberfäche verdunstet oder verdampft, muß durch die Poren in der festen Hülle an die Oberfläche diffundieren. Je nach Durchmesser der Poren gibt es unterschiedliche Regime. Bei großen Poren wird der Gasstrom nicht durch die Wände behindert und der Diffusionskoeffizient $D_{M}^{P}$ berechnet sich zu

$$
D_{M}^{P}=D \cdot \frac{1-\epsilon}{\tau},
$$

wobei die Indizes $M$ und $P$ die molekulare Diffusion bzw. Porendiffusion kennzeichnen. $1-\epsilon$ ist die Porosität und $\tau$ der sogenannte Tortuositätsfaktor. Er beschreibt das Verhältnis von zu durchströmender Körperlänge zum tatsächlich zurückzulegenden Weg [35]. Der Diffusionskoeffizient $D$ von Wasserdampf in Luft in Abhängigkeit von der Temperatur $T$ kann nach Fuller mit

$$
D=\frac{0,00143 T^{1,75}}{0,0120}
$$

berechnet werden [37]. Bei sehr engen Poren im Bereich der Knudsendiffusion berechnet sich der Diffusionskoeffizient $D_{K}^{P} \mathrm{zu}$

$$
D_{K}^{P}=\frac{d_{P}}{3} \cdot \sqrt{\frac{8 \cdot \Re \cdot T}{\pi \cdot M}} \cdot \frac{\epsilon}{\tau}
$$

wobei $\Re$ die allgemeine Gaskonstante, $M$ die molare Masse und $T$ die Temperatur darstellt. Da die Diffusion durch die Wände behindert wird, nimmt der Diffusionskoeffizient mit abnehmendem Porendurchmesser $d_{p}$ ab. Für den Übergangsbereich zwischen molekularer und Knudsen-Diffusion werden die beiden Diffusionskoeffizienten in Reihe geschaltet [35]:

$$
\frac{1}{D^{P}}=\frac{1}{D_{M}^{P}}+\frac{1}{D_{K}^{P}}
$$




\section{Kapitel 4}

\section{Grundlagen der Feststoffbildung}

Es gibt verschiedene Feststoffbildungsmechanismen. Feststoff kann sich durch Kristallisation, amorphe Erstarrung oder Polymerisation bilden. Das vorliegende Modell konzentriert sich auf die Berücksichtigung der Kristallisation als Feststoffbildungsprozeß. Prinzipiell ist es jedoch möglich, die Polymerisation oder die Erstarrung der gelösten Substanz zu einem amorphen Feststoff als Feststoffbildungsprozeß zu integrieren.

\section{1 Übersättigung}

Der Kristallisationsprozeß aus einer Lösung beginnt, sobald die Sättigungsgrenze überschritten wurde. Dies kann durch Kühlung der Lösung, Verdampfung des Lösungsmittels, durch die Reaktion zweier Komponenten zu einer schwerlöslichen Substanz oder das Hinzufügen einer weiteren Komponente, welche die Löslichkeit herabsetzt, erfolgen. In verdünnten Salzlösungen liegt das Salz vollständig ionisiert vor, wobei jedes Ion wegen der polaren Natur der Wassermoleküle von einer Hydrat-Hülle umgeben ist, die je nach Stärke und Reichweite der Wechselwirkung aus Regionen der Strukturbildung bzw. Strukturbrechung besteht. Eine weitere Hydrat-Hülle, die mit der Umgebung Wassermoleküle austauscht, ist locker mit der ersten verbunden. Mit zunehmender Konzentration der Lösung nimmt der Grad der Ionisierung ab, und die gelöste Substanz liegt zu einem großen Teil als Ionen-Paare vor. Mit zunehmender Konzentration bilden sich Cluster aus Salzgitterbausteinen aus, in denen die Gitterbausteine wie im späteren Kristall vorliegen. Diese Cluster sind jedoch noch zu klein, um als eigene Phase bezeichnet werden zu können. Übersättigte Lösungen weisen eine metastabile Zone auf, in welcher nur Wachstum stattfindet, jedoch keine Keimbildung. Die Breite dieser Zone hängt von der Bewegung, der Konzentration der Kristalle und dem Anteil weiterer gelöster Stoffe in der Lösung ab [42] (Abb. 4.1). 


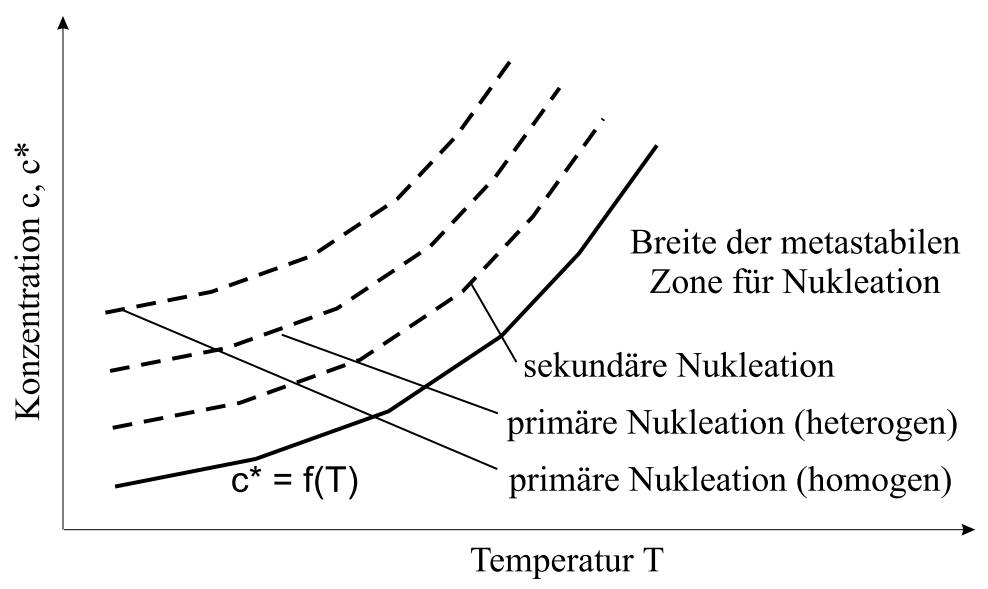

Abbildung 4.1: Dargestellung der Breite der metastabilen Zone in Abhängigkeit von der Temperatur $T$ nach [41] ( $c$ : Konzentration, $c^{*}$ : Löslichkeit)

\subsection{Keimbildung}

Bei der Kristallisation wird zwischen primärer und sekundärer Keimbildung unterschieden (Abb. 4.2). Primäre Keimbildung findet in einer homogenen Lösung statt, wohingegen bei sekundärer Kristallisation die Kristallisation durch mechanische Einwirkungen auf die Kristalle stattfindet.

Damit die Atome, Moleküle oder Ionen aus einer homogenen Lösung ein Kristallgitter bilden, ist Energie für die Bildung der Grenzfläche und der festen Phasen notwendig. Die Änderung der freien Enthalpie bei der Kristallisation setzt sich aus der Änderung der freien Enthalpie aufgrund der Oberflächenbildung $G_{S}$ und der Änderung aufgrund der Kristallentstehung $G_{V}$ zusammen [42].

$$
\Delta G=\Delta G_{S}+\Delta G_{V}
$$

Dabei ist die Enthalpieänderung $\Delta G_{S}$ bei der Oberflächenbildung positiv und die Änderung der freien Enthalpie durch den Phasenübergang $\Delta G_{S}$ negativ. Daraus ergibt sich die kritische Keimgröße. Dies ist der Keimdurchmesser, ab welchem ein entstandener Keim wächst, weil die Änderung der freien Enthalpie negativ ist. Keime, deren Durchmesser geringer ist, lösen sich wieder auf. Die kritische Keimgröße berechnet sich nach [41] zu

$$
L_{k r i t}^{*}=\frac{4 \sigma}{\left(1-k_{v}+\nu k_{v}\right) \Re T c_{c} \ln S}
$$

mit der Übersättigung $S=c / c_{s}$, dem Formfaktor $k_{v}$, der Oberflächenspannung $\sigma$, der Clusterkonzentration $c_{c}$ und der kinematischen Viskosität $\nu$. Je größer die Übersättigung $\mathrm{S}$ ist, desto geringer ist die kritische Keimgröße.

Bei der primären Kristallisation wird zwischen homogener und heterogener Kristallisation differenziert. Bei der homogenen Kristallisation entstehen Keime durch die Übersättigung der 


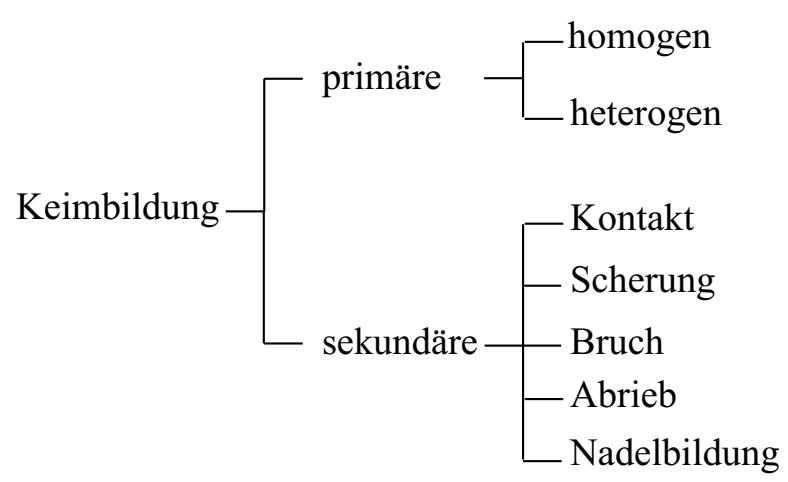

Abbildung 4.2: Verschiedene Keimbildungsmechanismen [42]

Lösung. Heterogene Kristallisation hingegen findet an Verunreinigungen oder Oberflächen des Kristallisators als Kristallisationskeimen statt. Ein Ansatz zur Berechnung der Keimbildungsrate bei der primären homogenen Keimbildung ist

$$
B^{h o m}=C \exp \left(\frac{16 \pi \sigma^{3} \nu^{2}}{3 k^{3} T^{3}(\ln S)^{2}}\right)
$$

mit k als Boltzmannkonstante. Dieser Ausdruck kann auch durch

$$
B^{h o m}=k_{B}\left(c-c_{m}\right)^{n_{b}}
$$

angenähert werden, wobei $c_{m}$ die Konzentration am Rand des metastabilen Bereichs und $k_{b}$ der Nukleationsfaktor ist. Bei den meisten anorganischen Systemen ist $c_{m}$ jedoch dicht bei der Sättigungskonzentration $c_{s}$, so daß auch

$$
B^{h o m}=k_{B}\left(c-c_{s}\right)^{n_{b}}
$$

geschrieben werden kann [42]. Für die heterogene Keimbildung ist das folgende Modell [42] vorgeschlagen worden:

$$
B^{\text {het }}=B^{\text {hom }} \exp \left(\frac{16 \pi \sigma^{3} \nu^{2}}{3 k^{3} T^{3}(\ln S)^{2}}\right) .
$$

Die größte Quelle für neue Keime bei der sekundären Keimbildung ist die Kontaktnukleation. Nach einem Stoß senkrecht zur Oberfläche, welcher den Kristall nicht beschädigt, entstehen neue Kristalle. Die Keimbildung ist dabei auf eine an der Kristalloberfläche adsorbierte Schicht noch nicht kristallisierter gelöster Substanz zurückzuführen. Bei höherer Übersättigung entstehen mehr Keime, weil die Schicht dicker und die kritische Keimgröße geringer ist. Die Keimbildung findet dabei nur an wachsenden Kristallen statt [42].

\subsection{Kristallwachstum}

Das Kristallwachstum ist ein sehr komplexer Prozeß. Generell wird zwischen diffusionskontrolliertem und einbaukontrolliertem Wachstum unterschieden. Die Cluster und Moleküle diffun- 
dieren zunächst zur Oberfläche des Kristalls und werden dort anschließend in die Oberfläche eingebaut. Bei diffusionskontrolliertem Wachstum wird die Wachstumsgeschwindigkeit von der Diffusion der Ionen durch die Grenzschicht bestimmt, und die Wachstumsrate läßst sich mit Hilfe einer Massentransportgleichung beschreiben. Die Wachstumsgeschwindigkeit wird durch die Geschwindigkeit der Übersättigungszunahme relativ zur Kristalloberfläche gesteigert. Ist dies nicht mehr der Fall, wird die Wachstumsgeschwindigkeit durch den Einbauschritt des Ions in die Oberfäche bestimmt. An der Oberfläche des Kristalls bildet sich eine "dritte Phase", die aus gelöster Substanz mit teilweise geordneter Struktur besteht. Für den Integrationsschritt gibt es verschiedene Modelle. Das kontinuierliche Wachstumsmodell (Abb. 4.3, (a)) nimmt eine raue Oberfläche an, in die neue Bauteile eingebaut werden, wenn dadurch ihre freie Enthalpie abnimmt. Beim Oberflächen-Keimbildungs-Wachstums-Modell (Abb. 4.3, (b)) ist das Wachstum durch die Frequenz der Bildung von zweidimensionalen Keimen an einer glatten Oberfläche bestimmt. Der bestimmende Schritt ist die Keimbildung, während die nachfolgenden Schritte auf Grund der geringeren notwendigen Energie sehr viel schneller sind. Das am meisten verbreitete Modell ist das BCF-Modell (Burton-Canberra-Frank) (Abb. 4.3, (c)). Bei diesem Modell wird durch die Einbindung einer Wachstums-Einheit eine Rille gefüllt und gleichzeitig eine neue geschaffen, die das Wachstum begünstigt. Dies führt zu Schraubenversetzungen. Bei der BCF-Theorie ist die Wachstumsgeschwindigkeit von der Übersättigung der Lösung abhängig. Die Wachstumsgeschwindigkeit wird dabei aus der Gleichung

$$
G=\frac{d L}{d t}=k_{G}\left(c-c_{s}\right)
$$

berechnet mit

$$
k_{G}=\frac{\beta k_{v}}{3 k \nu \rho},
$$

wenn das Wachstum sowohl durch die Diffusion als auch durch den Einbau bestimmt wird [42].

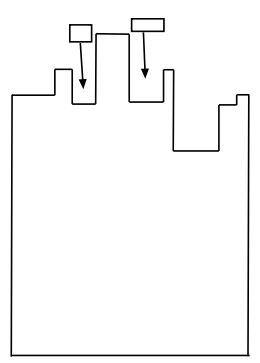

(a)

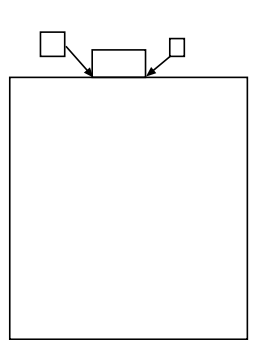

(b)

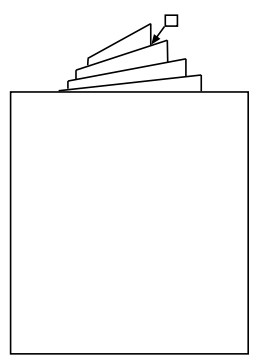

(c)

Abbildung 4.3: Modelle für das Kristallwachstum: (a) Kontinuierliches Wachstum, (b) Oberflächen-Keimbildungs-Wachstums-Modell, (c) Schraubenversetzungen (BCF-Modell) [42] 


\subsection{Kristallisation im Tropfen}

Im Tropfen ist davon auszugehen, daß die Feststoffbildung auf Grund von sowohl homogener wie heterogener Kristallisation stattfindet. Durch das Zerstäuben der Lösung werden Tropfen erzeugt, die alle die gleiche Konzentration gelöster Substanz haben. Durch die je nach Umgebungsbedingung vorhandenen unterschiedlichen Mengen an Verunreinigungen in der Lösung kommt es zu homogener oder heterogener Keimbildung. Da im Tropfen keine Strömung vorhanden ist und somit auch keine großen Kräfte auf die entstehenden Kristalle wirken, kann sekundäre Kristallisation ausgeschlossen werden. 


\section{Kapitel 5}

\section{Mathematisches Modell}

Um die Feststoffbildung simulieren zu können, müssen die zeitliche Änderung des Massenanteils der gelösten Substanz und der Temperatur im Tropfen berechnet werden. Temperatur und Dampfpartialdruckdifferenz zwischen der Tropfenoberfläche und dem Trocknungsgas bestimmen die Geschwindigkeit des Schrumpfungsprozesses, durch den sich die gelöste Substanz aufkonzentriert. Von der Verteilung der gelösten Substanz hängt wiederum die Kristallisation ab. Sobald sich eine feste Hülle gebildet hat, muß das Modell das Wachsen der Hülle simulieren können.

Das mathematische Modell ist eindimensional und radialsymmetrisch. Die Differentialgleichungen werden mit einem Finite-Differenzen- und einem Finite-Volumen-Verfahren dikretisiert und gekoppelt gelöst.

Im Folgenden werden zunächst die Gleichungen für den 1. Trocknungsabschnitt dargestellt. Anschließend wird auf die Modellierung des 2. Trocknungsabschnitts eingegangen. Abb. 5.1 veranschaulicht das mathematische Modell im 1. Trocknungsabschnitt.

\subsection{Bewegliche Phasengrenzen}

Während des Trocknungsprozesses bewegen sich die Phasengrenzen des Tropfens. Es ist daher notwendig, die zeitliche Änderung des Ortes der Phasengrenze zu verfolgen. Im vorliegenden Fall wird die Bewegung der Phasengrenze durch eine Normierung des Radius $r$ berücksichtigt, wodurch sich die Phasengrenze immer auf dem selben Diskretisierungspunkt befindet. Dadurch ist keine weitere Gleichung zur Bestimmung des Ortes der Phasengrenze, wie im Falle eines festen Koordinatensystems, notwendig. Auch kann bei einem festen Koordinatensystem die Phasengrenze zwischen zwei Punkten des Koordinatensystems liegen, was die Lösung der Gleichungen erschwert. Zur Normierung wird die lineare Beziehung

$$
\tilde{r}=\frac{r-r_{i}(t)}{r_{a}(t)-r_{i}(t)}
$$




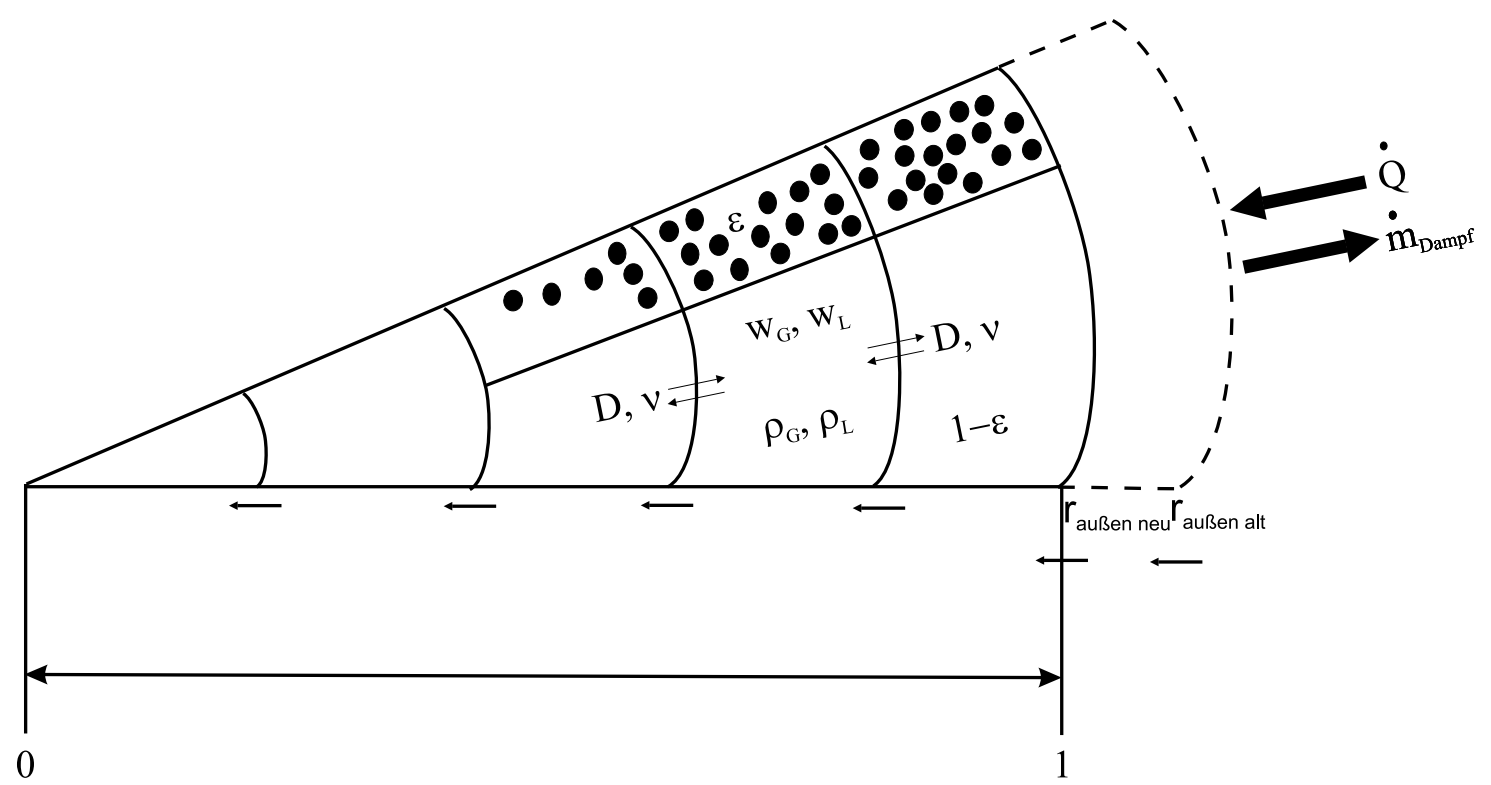

Abbildung 5.1: Mathematisches Modell des Trocknungsprozesses: Dem Tropfen wird der Wärmestrom $\dot{Q}$ zugeführt. Durch die Verdunstung wird der Lösunsgmittelmassenstrom $\dot{m}$ abgeführt. Der Stofftransport im Tropfen findet durch Diffusion $D$ und Konvektion $v$ statt. Der Wärmetransport erfolgt durch Wärmeleitung $\lambda$ und Massentransport. Die Partikel nehmen den Volumenanteil $\epsilon$ ein.

verwendet, wobei die Indizes $a$ und $i$ den Tropfenrand und den Tropfenmittelpunkt kennzeichnen. Die Normierung hat eine Transformation der Bilanzgleichungen zur Folge.

$$
\left(\frac{\partial}{\partial t}\right)_{r}=\left(\frac{\partial}{\partial \tilde{t}}\right)_{\tilde{r}} \frac{d \tilde{t}}{d t}+\frac{\partial}{\partial \tilde{r}} \frac{\partial \tilde{r}}{\partial t}
$$

$\tilde{t}$ ist dabei die transformierte Zeit, wobei jedoch im vorliegenden Fall $t=\tilde{t}$ gilt, so daß

$$
\left(\frac{\partial}{\partial t}\right)_{r}=\left(\frac{\partial}{\partial \tilde{t}}\right)_{\tilde{r}}+\frac{\partial}{\partial \tilde{r}} \frac{\partial \tilde{r}}{\partial t}
$$

und sich somit

$$
\left(\frac{\partial}{\partial t}\right)_{r}=\left(\frac{\partial}{\partial \tilde{t}}\right)_{\tilde{r}}-\frac{1}{r_{a}(t)-r_{i}(t)} \frac{\partial}{\partial \tilde{r}} \cdot\left[\frac{d r_{i}(t)}{d t}+\tilde{r}\left(\frac{d r_{a}(t)}{d t}-\frac{d r_{i}(t)}{d t}\right)\right]
$$

ergibt [43].

\subsection{Stofftransport}

Der Stofftransport im Tropfen wird mit Fickscher Diffusion und Konvektion berechnet. Darüber hinaus wird in der Massenbilanz der Phasenübergang der gelösten Substanz durch Kristallisation berücksichtigt. Die zeitliche Änderung der radialen Konzentrationsverteilung wird 
also mit

$$
\begin{gathered}
\frac{\partial\left(\rho w_{G}(1-\epsilon)\right)}{\partial t}=\underbrace{-\frac{1}{r^{2}} \frac{\partial\left(r^{2} \rho w_{G} v(1-\epsilon)\right)}{\partial r}}_{\text {Konvektion }} \\
\underbrace{+\frac{1}{r^{2}} \frac{\partial}{\partial r}\left(r^{2} D \rho(1-\epsilon) \frac{\partial w_{G}}{\partial r}\right)}_{\text {Diffusion }} \underbrace{-\rho_{G}^{\prime} \frac{\pi}{6} \int_{L_{\min }}^{L_{\max }} G \frac{\partial n}{\partial L} L^{3} d L}_{\text {Feststoffbildung }}
\end{gathered}
$$

berechnet [44]. Durch die Transformation mit Gl. (5.1) ergibt sich analog zu Gl. (5.3):

$$
\begin{gathered}
\frac{\partial\left(\rho w_{G}(1-\epsilon)\right)}{\partial t}=\underbrace{-\frac{1}{r_{a}-r_{i}} \frac{1}{\left(r_{i}+\tilde{r}\left(r_{a}-r_{i}\right)\right)^{2}} \frac{\partial\left(\left(r_{i}+\tilde{r}\left(r_{a}-r_{i}\right)\right)^{2} \rho w_{G} v(1-\epsilon)\right)}{\partial \tilde{r}}}_{\text {Konvektion }} \\
\quad \underbrace{+\frac{1}{\left(r_{a}-r_{i}\right)^{2}} \frac{1}{\left(r_{i}+\tilde{r}\left(r_{a}-r_{i}\right)\right)^{2}} \frac{\partial}{\partial \tilde{r}}\left(\left(r_{i}+\tilde{r}\left(r_{a}-r_{i}\right)\right)^{2} D \rho(1-\epsilon) \frac{\partial w_{G}}{\partial \tilde{r}}\right)}_{\text {Feststoffbildung }} \\
\underbrace{+\frac{\partial\left(\rho w_{G}(1-\epsilon)\right)}{\partial \tilde{r}} \frac{1}{r_{a}-r_{i}}\left[\frac{d r_{i}}{d t}+\tilde{r}\left(\frac{d r_{a}}{d t}-\frac{d r_{i}}{d t}\right)\right]}_{-\rho_{G}^{\prime} \frac{\pi}{6} \int_{L_{\text {min }}}^{L_{\max }} G \frac{\partial n}{\partial L} L^{3} d L} .
\end{gathered}
$$

Da ein Zweikomponentensystem berechnet wird, errechnet sich der Massenanteil des Lösungsmittels aus $w_{L}=1-w_{G} \cdot \epsilon$ stellt den Volumenanteil der festen Phase dar, wobei der Massenanteil in der zeitlichen Ableitung mit dem Flüssigkeitsvolumenanteil $(1-\epsilon)$ multipliziert werden muß, um ihn auf das Volumen der flüssigen Lösung zu beziehen [45]. In den Diffusions- und Konvektionstermen wird mit $(1-\epsilon)$ die zum Stofftransport zur Verfügung stehende Fläche reduziert. Der im Tropfen stattfindende diffusive Stofftransport mit dem Diffusionskoeffizienten $\mathrm{D}$ wird durch den zweiten Term auf der rechten Seite von Gl.(5.6) beschrieben. $\rho$ ist dabei die Gesamtdichte. Wegen der unterschiedlichen Dichten $\rho^{\prime}$ der beiden diffundierenden Komponenten muß ein konvektiver Ausgleichsstrom berücksichtigt werden, der durch den ersten Term der rechten Seite von Gl.(5.6) beschrieben wird. Die Geschwindigkeit $v$ des konvektiven Stofftransportes ergibt sich aus der Bedingung, daß die Volumenströme gleich groß sind,

$$
\sum_{i} \dot{V}_{i}=\frac{\dot{m}_{G}}{\rho_{G}^{\prime}}+\frac{\dot{m}_{L}}{\rho_{L}^{\prime}}=0
$$

weil die Flüssigkeit als ideal und inkompressibel betrachtet wird. Der konvektive Massenstrom $\dot{m}$ hat daher die Geschwindigkeit:

$$
\begin{gathered}
-\frac{\rho(1-\epsilon)}{\rho_{G}^{\prime}} v w_{G}+\frac{\rho(1-\epsilon)}{\rho_{G}^{\prime}} D \frac{\partial w_{G}}{\partial r}-\frac{\rho(1-\epsilon)}{\rho_{L}^{\prime}} v w_{L}+\frac{\rho(1-\epsilon)}{\rho_{L}^{\prime}} D \frac{\partial w_{L}}{\partial r}=0 \\
v=D \frac{\partial w_{G}}{\partial r} \frac{\frac{1}{\rho_{G}^{\prime}}-\frac{1}{\rho_{L}^{\prime}}}{\frac{w_{L}}{\rho_{L}^{\prime}}+\frac{w_{G}}{\rho_{L}^{\prime}}} .
\end{gathered}
$$


Damit die Konzentrationsverteilung am selben Ort bleibt, während das Koordinatensystem sich unter ihr hinwegbewegt, kommt durch die Koordinatentransformation der dritte „pseudokonvektive“ Term hinzu. Im Term, der die Kristallisation beschreibt, bezeichnet $n$ die Partikelanzahldichte, $L$ die Partikelgröße und $G$ die Wachstumsgeschwindigkeit der Kristalle. Eine Erläuterung des Terms findet im Rahmen von Kapitel 5.4 statt. Am Tropfenrand ergibt sich der Gradient des Massenanteils für das Lösungsmittel aus der Massenbilanz um die Phasengrenze, d.h. die im Tropfen an die Phasengrenze transportierte Stoffmenge ist gleich der verdampften:

$$
\dot{m}=\rho v w_{L}-\left.\rho D \frac{\partial w_{L}}{\partial r}\right|_{r=r_{a}} .
$$

Da die gelöste Substanz nicht verdampft, lautet die Randbedingung für die gelöste Substanz

$$
\dot{m}=0=\rho v w_{G}-\left.\rho D \frac{\partial w_{G}}{\partial r}\right|_{r=r_{a}} .
$$

Der Dampfmassenstrom

$$
\dot{m}=\beta \frac{p_{O}-p_{U}}{R_{D} T}
$$

berechnet sich aus der Differenz der Dampfpartialdrücke zwischen Tropfenoberfläche $p_{O}$ und Trocknungsgas $p_{U}$ mit $R_{D}$ als spezieller Gaskonstante und T als Temperatur. Den Stoffübergangskoeffizienten $\beta$ in Gl. (5.11) erhält man aus der Sherwood-Zahl

$$
S h=\frac{\beta \cdot d_{\text {Tropfen }}}{D_{\text {Gas }}}=2+0,6 \cdot \sqrt{R e} \cdot S c^{0,33},
$$

worin $d_{\text {Tropfen }}$ den Tropfendurchmesser und $D_{\text {Gas }}$ den Diffusionskoeffizienten von Wasserdampf in Luft darstellt [35]. Wegen der geringen Relativgeschwindigkeit des Tropfens (siehe Kapitel 3.3) ist die Reynolds-Zahl sehr klein und somit $S h \approx 2$. Im Inneren des Tropfens gilt aufgrund der radialsymmetrischen Berechnung als Regularitätsbedingung:

$$
\left.\frac{\partial w_{G}}{\partial r}\right|_{r=0}=\left.\frac{\partial w_{L}}{\partial r}\right|_{r=0}=0
$$

Die Bewegung des Tropfenradius errechnet sich aus dem Dampfmassenstrom, der den Tropfen aufgrund der Verdunstung verläßt:

$$
\frac{d r}{d t}=\frac{\dot{m}_{\text {Dampf }}}{\rho_{\text {Dampf }}}
$$

\subsection{Temperatur des Tropfens}

In der flüssigen Phase des Tropfens errechnet sich die Temperaturverteilung aus der Fourierschen Differentialgleichung. Da jedoch im Tropfen auch Stofftransport stattfindet, muß neben 
der Wärmeleitung der Enthalpietransport aufgrund des Stofftransports berücksichtigt werden [46]. Nach Transformation mit Gl. (5.1) ergibt sich

$$
\begin{aligned}
& \frac{\partial(\rho h)}{\partial t}=\underbrace{-\frac{1}{r_{a}-r_{i}} \frac{1}{\left(r_{i}+\tilde{r}\left(r_{a}-r_{i}\right)\right)^{2}} \frac{\partial\left(\left(r_{i}+\tilde{r}\left(r_{a}-r_{i}\right)\right)^{2} \dot{m}_{G} h_{G}+\dot{m}_{L} h_{L}\right)}{\partial \tilde{r}}}_{\text {Massentransport }} \\
& \underbrace{+\frac{1}{\left(r_{a}-r_{i}\right)^{2}} \frac{1}{\left(r_{i}+\tilde{r}\left(r_{a}-r_{i}\right)\right)^{2}} \frac{\partial}{\partial \tilde{r}}\left(\left(r_{i}+\tilde{r}\left(r_{a}-r_{i}\right)\right)^{2} \lambda \frac{\partial T}{\partial \tilde{r}}\right)}_{\text {Leitung }} \\
& \underbrace{+\frac{\partial(\rho h)}{\partial \tilde{r}} \frac{1}{r_{a}-r_{i}}\left[\frac{d r_{i}}{d t}+\tilde{r}\left(\frac{d r_{a}}{d t}-\frac{d r_{i}}{d t}\right)\right]}_{\text {'Pseudo-Konvektion" }}, \\
& \text { wobei } h_{i}=\int_{T_{r e f}}^{T} c_{p i} d T \\
& \text { und } h=\sum_{i=1}^{n} w_{i} \int_{T_{r e f}}^{T} c_{p i} d T \text {. }
\end{aligned}
$$

Der Energietransport aufgrund der Massenströme wird durch den ersten Term von Gl.(5.15) beschrieben mit $h$ als Enthalpie und der Energietransport aufgrund der Leitung durch den zweiten mit $\lambda$ als Wärmeleitfähigkeit und $T$ als Temperatur. Die Koordinatentransformation wird durch den dritten Term berücksichtigt. Durch Integration der Wärmekapazitäten $c_{p i}$ errechnen sich die Enthalpien. Kristallisationswärme wird nicht berücksichtigt, weil bei experimentellen Untersuchungen von Lin und Gentry über den Einfluß der Krsitallisationswärmen auf den Trocknungsprozeß von Einzeltropfen keine eindeutigen Zusammenhänge festgestellt werden konnten [47]. Die Randbedingung am Tropfenrand erhält man aus der Energiebilanz um die Phasengrenze, d.h. daß die an den Tropfen aus der Umgebung übertragene Energie gleich der im Tropfen weitergeleiteten sein muß, abzüglich der mit dem Dampf abtransportierten Enthalpie $h_{v}$ :

$$
\left.\lambda \frac{\partial T}{\partial r}\right|_{r=r_{a}}=\alpha\left(T_{\text {Umgebung }}-T_{\text {Wand }}\right)-h_{v} \dot{m} .
$$

Der Wärmeübergangskoeffizient $\alpha$ ergibt sich aus der Nusseltzahl [35]:

$$
N u=\frac{\alpha \cdot d_{\text {Tropfen }}}{\lambda_{\text {Gas }}}=2+0,6 \cdot \sqrt{R e} \cdot \operatorname{Pr}^{0,33} .
$$

Wegen der geringen Relativgeschwindigkeit des Tropfens gilt für die Nusselt-Zahl analog zur Sherwood-Zahl $N u \approx 2$. Im Tropfenmittelpunkt gilt wegen der radialsymmetrischen Berechnung die Regularitätsbedingung

$$
\left.\lambda \frac{\partial T}{\partial r}\right|_{r=0}=0
$$




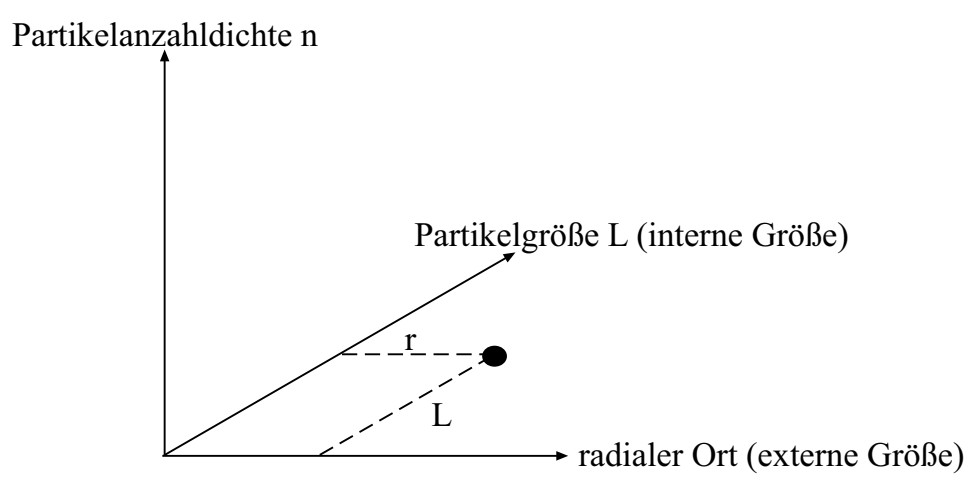

Abbildung 5.2: Die Populationsbilanz hat zwei Dimensionen, die Partikelgröße L und die Lage auf dem Tropfenradius $\mathrm{r}$

\subsection{Modellierung der Feststoffbildung mit Populationsbilanzen}

Die Feststoffbildung im Tropfen wird mit Hilfe von Populationsbilanzen berechnet. In ihrer allgemeinen Form lautet die Populationsbilanz

$$
\frac{\partial n}{\partial t}+\nabla v_{i} n+\nabla v_{e} n+B+D=0 .
$$

Durch den ersten Term wird die zeitliche Änderung der Partikelanzahldichte $n$ ausgedrückt. Vorgänge in einem internen Koordinatensystem, das Partikeleigenschaften wie z.B. Partikelgröße oder den Agglomerationsgrad beschreibt, werden durch den zweiten Term dargestellt. Mit dem dritten Term werden räumliche Bewegungen der Partikel in einem externen Koordinatensystem (z.B. im flüssigen Tropfen) berücksichtigt. Abb. 5.2 veranschaulicht die zwei Dimensionen der Populationsbilanz. Der Birth-Term $B$ und der Death-Term $D$ beschreiben die Entstehung von Partikeln durch Bruch oder Agglomeration von Partikeln. Im Fall der Partikel im Tropfen wird ausschließlich das Wachstum der Partikel berechnet, weil Bruch im Tropfen nicht stattfindet. Weiterhin wird angenommen, daß die Partikel bis zum Einbau in der Hülle unagglomeriert vorliegen, das heißt die Partikel agglomerieren ausschließlich im zweiten Trocknungsabschnitt an der festen Hülle. Es ergibt sich also

$$
\frac{\partial n}{\partial t}=\underbrace{-\frac{\partial(G n)}{\partial L}}_{\text {Wachstum }} \underbrace{+\frac{\partial n}{\partial \tilde{r}} \frac{1}{r_{a}-r_{i}}\left[\frac{d r_{i}}{d t}+\tilde{r}\left(\frac{d r_{a}}{d t}-\frac{d r_{i}}{d t}\right)\right]}_{\text {"Pseudo-Konvektion" }} .
$$

Die Gleichung hat zwei Dimensionen, den Radius $r$ und die Partikellänge L (Abb. 5.2). Der erste Term von Gl.(5.20) beschreibt das Wachstum der Partikel mit der Geschwindigkeit G. Durch den zweiten Term wird beschrieben, daß die Partikel am Ort ihrer Entstehung bleiben, während sich das Koordinatensystem unter ihnen hinweg bewegt (Abb. 5.3). Dies führt zu einer Konzentration der Partikel am Tropfenrand, weil die Partikel an der Phasengrenze von dieser mittransportiert werden. In der Partikellängenkoordinate gilt bei der kleinsten 


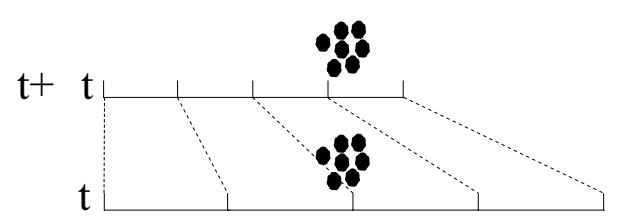

Abbildung 5.3: Beispielhafte Darstellung der Bewegung des Koordinatensystems unter den Partikeln hinweg in Abhängigkeit von der Zeit, während die Partikel am selben Ort bleiben.

Partikelgrößenklasse als Randbedingung

$$
n_{0}=\frac{B_{0}}{G}
$$

mit der Keimbildungsrate $B$. Diese Randbedingung ergibt sich nach [41] aus

$$
B_{0}=\frac{d N_{0}}{d t}=\frac{d N_{0}}{d L} \frac{d L}{d t}=n_{0} G .
$$

Die größte Partikelklasse muß so gewählt werden, daß die Partikel diese Länge nicht erreichen:

$$
n_{\max }=0
$$

Für die örtliche Ableitung gilt im Tropfenmittelpunkt wegen der radialsymmetrischen Rechnung

$$
\left.\frac{\partial n}{\partial r}\right|_{r=r_{i}}=0
$$

Am Tropfenrand ist die Festlegung einer Randbedingung schwieriger. Die Partikel verlassen den flüssigen Tropfen nicht, und es ist daher die „Aufkonzentration“ der Partikel in der äußersten Schale mathematisch zu beschreiben. Als Randbedinung für die örtliche Ableitung am Tropfenrand gilt daher

$$
\dot{n}=0 \text {. }
$$

Die mathematische Ausführung der rechten Randbedingung ist in Kapitel 6.2.2 beschrieben. Da die Partikel am Rand den Tropfen nicht verlassen, muß die Gleichung zunächst für den Tropfenrand modifiziert werden. In Gleichung (5.20) muß ein Term hinzugefügt werden, der die relative Volumenänderung des Kontrollvolumens beschreibt. Die Gleichung hat dann die Form

$$
\frac{\partial n}{\partial t}=-\frac{\partial(G n)}{\partial L}+\frac{\partial n}{\partial \tilde{r}} \frac{1}{r_{a}-r_{i}}\left[\frac{d r_{i}}{d t}+\tilde{r}\left(\frac{d r_{a}}{d t}-\frac{d r_{i}}{d t}\right)\right]-\frac{3}{r_{a}} \frac{d r_{a}}{d t} n(r, L)
$$

Da die sich im Tropfen ausbildende Hülle dicker als nur ein Kontrollvolumen ist, muß Gl. (5.26) weiter in das Innere des Tropfens verschoben werden. In den Zellen, die weiter innen liegen, gilt Gl. (5.20) und in den weiter außen liegenden

$$
\frac{\partial n}{\partial t}=-\frac{\partial(G n)}{\partial L}+\frac{3}{r_{a}} \frac{d r_{a}}{d t} n(r, L) \text {. }
$$


Die mathematische Beschreibung der physikalischen Vorgänge auf diese Weise ist jedoch nicht optimal, weil die Zahl der Diskretisierungspunkte und ihr Abstand die Anzahldichteverteilung im Tropfen mitbestimmen. Durch die Schrumpfung des Tropfens und die Abnahme der Kontrollvolumina dürfte sich die Partikelkonzentration in den Zellen nicht erhöhen. Dies ließe sich dadurch erreichen, daß jedes Partikel im äußeren Bereich des Tropfens sich mit der Schrumpfungsgeschwindigkeit des Tropfens nach innen bewegt. Die Gleichung hätte dann die Form

$$
\begin{aligned}
\frac{\partial n}{\partial t}=-\frac{\partial(G n)}{\partial L}- & \frac{1}{\left(r_{a}-r_{i}\right)^{2}} \frac{1}{\left(r_{i}+\tilde{r}\left(r_{a}-r_{i}\right)\right)^{2}} \frac{\partial}{\partial \tilde{r}}\left(\left(r_{i}+\tilde{r}\left(r_{a}-r_{i}\right)\right)^{2} n \frac{d r_{a}}{d t}\right) \\
& +\frac{\partial n}{\partial \tilde{r}} \frac{1}{r_{a}-r_{i}}\left[\frac{d r_{i}}{d t}+\tilde{r}\left(\frac{d r_{a}}{d t}-\frac{d r_{i}}{d t}\right)\right] .
\end{aligned}
$$

In den inneren Bereichen hätten die Partikel keine konvektive Geschwindigkeit. An der Schnittstelle zwischen den Bereichen würden die Partikel aufgesammelt. Die erstgenannte mathematische Methode ist jedoch zur Beschreibung verwendet worden, weil sie numerisch einfacher zu handhaben ist.

Der Volumenanteil der festen Phase wird aus

$$
\epsilon=\frac{\pi}{6} \int_{L_{\min }}^{L_{\max }} n L^{3} d L
$$

berechnet und die Änderung des Volumenanteils der festen Phase im Tropfen aus

$$
\frac{\partial \epsilon}{\partial t}=\frac{\pi}{6} \int_{L_{\min }}^{L_{\max }} \frac{\partial n}{\partial t} L^{3} \mathrm{~d} L .
$$

\subsubsection{Partikeldiffusion}

Wegen des radialen Konzentrationsgefälles der Partikel im Tropfen besteht die Möglichkeit, daß die Partikel im Tropfen diffundieren. Der Diffusionskoeffizient der Partikel kann nach der Gleichung von Stokes-Einstein berechnet werden [48],

$$
D_{S E}=\frac{k T}{6 \pi r \eta}
$$

worin $k$ die Boltzmannkonstante, $T$ die Temperatur, $\mathrm{r}$ den Partikelradius und $\eta$ die dynamische Viskosität darstellen. Abb. 5.4 zeigt Diffusionskoeffizienten für verschiedene Partikelgrößen bei $20^{\circ} \mathrm{C}$. Salzlösungen haben einen Diffusionskoeffizienten im Bereich von $10^{-9} \mathrm{~m}^{2} / \mathrm{s}$ [41]. Da jedoch der Diffusionskoeffizient für die größeren Partikel sehr klein ist im Verhältnis zum Diffusionskoeffizienten von Lösungen, kann die Partikeldiffusion vernachlässigt werden. Für sehr kleine Partikel/Keime ist der nach der Gleichung von Stokes-Einstein berechnete Diffusionkoeffizient sehr viel größer als für Salzlösungen. Dies läßt sich darauf zurückführen, daß die Gleichung nur für makroskopische Systeme gilt [37]. 


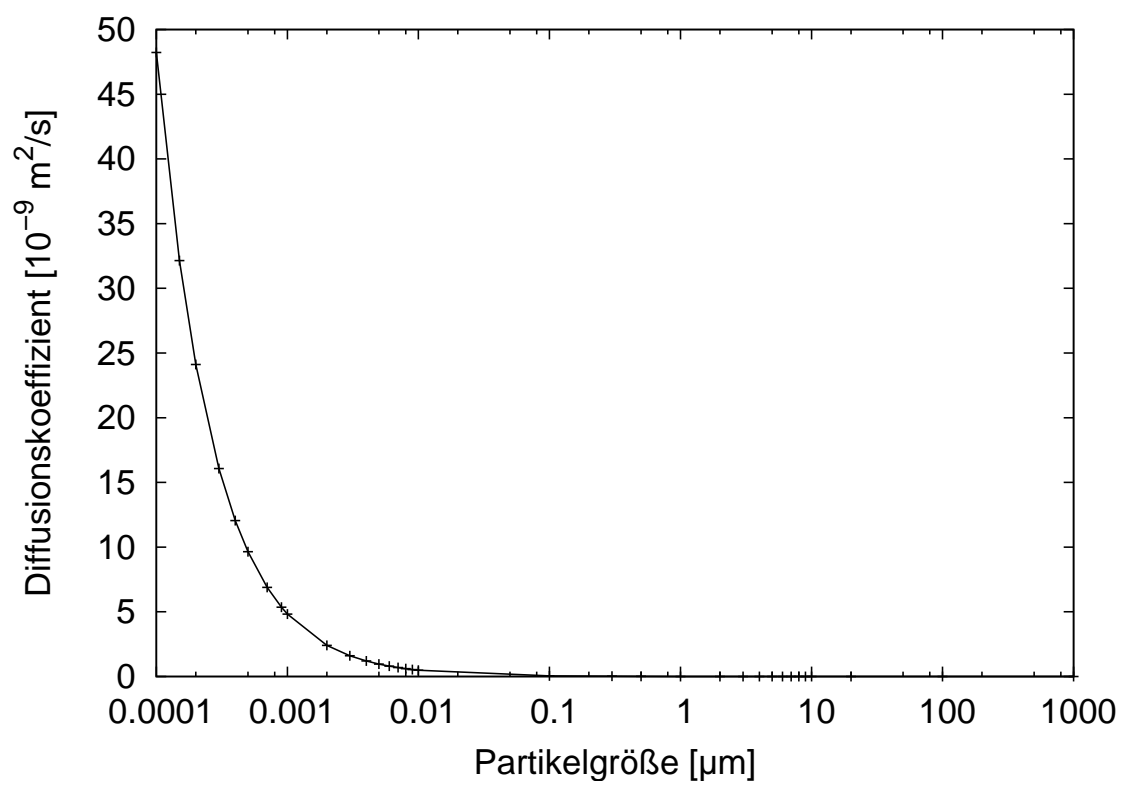

Abbildung 5.4: Stokes-Einstein-Diffusionskoeffizient der Partikel im Tropfen bei $293 \mathrm{~K}$ in Abhängigkeit von der Partikelgröße

\subsection{Modellierung des 2. Trocknungsabschnitts}

Im zweiten Trocknungsabschnitt hat sich eine feste Hülle ausgebildet, und der Tropfen schrumpft daher nicht mehr. Die feste Hülle wächst jedoch aufgrund der fortschreitenden Verdunstung/ Verdampfung des Lösungsmittels und der Kristallisation weiter nach innen. Es sind zwei Wege versucht worden, um das Wachsen der Hülle zu simulieren: (i) Die Hülle kann durch ein weiteres Koordinatensystem dargestellt werden, das außen an das bestehende Koordinatensystem anschließst (Abb. 5.5) und (ii) das bestehende Koordinatensystem wird in zwei Abschnitte aufgeteilt. Im äußeren Teil, welcher die Hülle darstellt, werden die Punkte auseinander gezogen, und im inneren Teil, welcher den flüssigen Kern darstellt, werden die Punkte zusammengedrückt. In den folgenden beiden Kapiteln werden die beiden unterschiedlichen Wege der Modellierung beschrieben, wobei die numerische Lösung in Kap. 6.5 erläutert wird. Für beide Modellierungswege wird angenommen, daß sich keine Flüssigkeit mehr in der Hülle befindet. Experimentelle Untersuchungen haben gezeigt, daß im 2. Trocknungsabschnitt das Lösungsmittel sowohl durch Dampfdiffusion als auch durch kapillaren Flüssigkeitstransport an die Oberfläche transportiert wird [49]. Zur Vereinfachung wird hier jedoch der kapillare Flüssigkeitstransport vernachlässigt. 


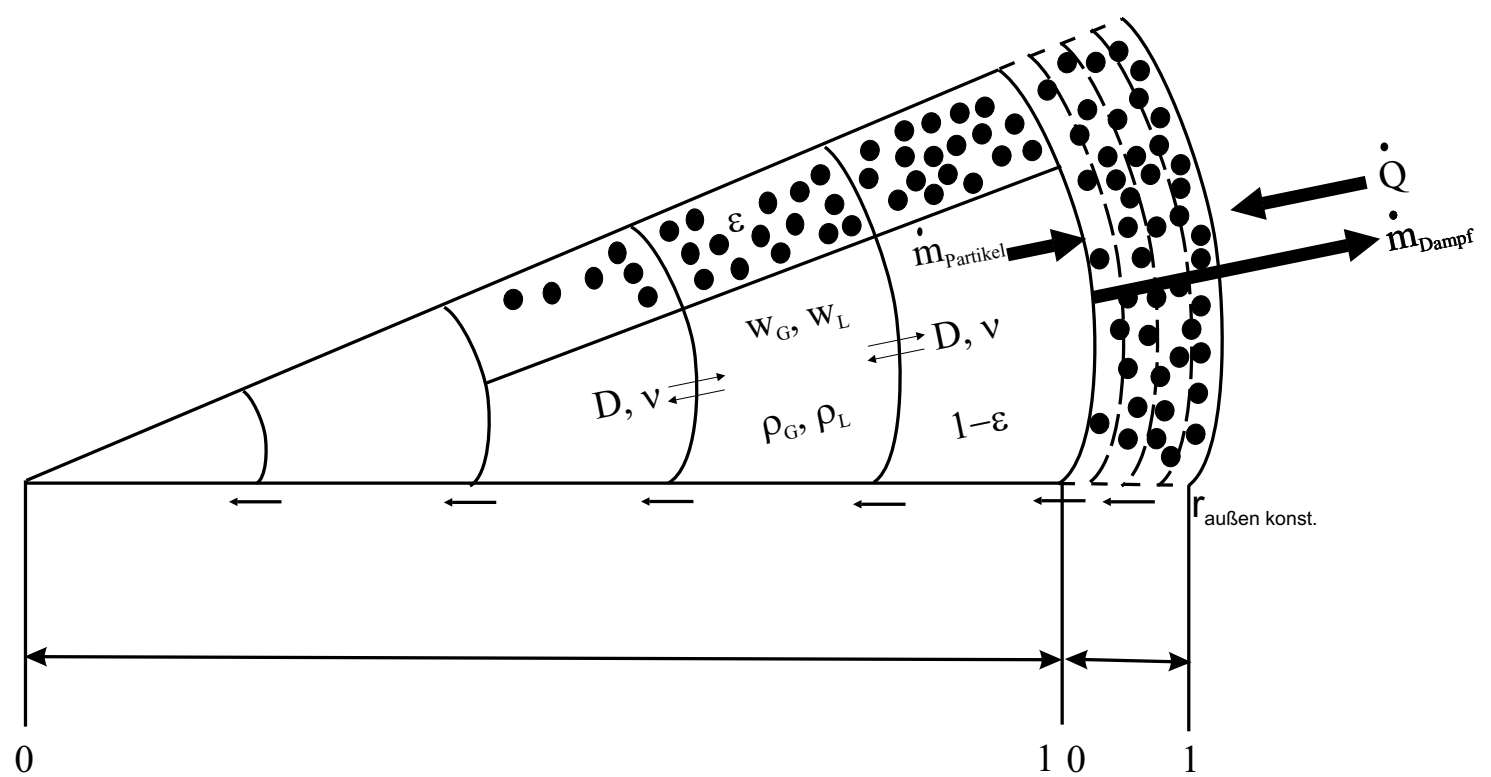

Abbildung 5.5: Mathematisches Modell des 2. Trocknungsabschnitts mit zwei Koordinatensystemen: Das innere normierte Koordinatensystem beschreibt den flüssigen Kern und das äußere die feste Hülle.

\subsubsection{Simulation des mehrschaligen Tropfens mit zwei Koordinatensyste- men}

\section{Temperatur des Tropfens}

In der Hülle wird ebenfalls der zeitliche radiale Temperaturverlauf berechnet. Hier findet der Wärmetransport jedoch ausschließlich durch Wärmeleitung statt:

$$
\begin{gathered}
\frac{\partial(\rho h)}{\partial t}=\underbrace{\frac{1}{\left(r_{a}-r_{i}\right)^{2}} \frac{1}{\left(r_{i}+\tilde{r}\left(r_{a}-r_{i}\right)\right)^{2}} \frac{\partial}{\partial \tilde{r}}\left(\left(r_{i}+\tilde{r}\left(r_{a}-r_{i}\right)\right)^{2} \lambda \frac{\partial T}{\partial \tilde{r}}\right)}_{\text {Leitung }} \\
\underbrace{+\frac{\partial(\rho h)}{\partial \tilde{r}} \frac{1}{r_{a}-r_{i}}\left[\frac{d r_{i}}{d t}+\tilde{r}\left(\frac{d r_{a}}{d t}-\frac{d r_{i}}{d t}\right)\right]}_{\text {'Pseudo-Konvektion" }}, \\
\text { wobei } h=\int_{T_{\text {ref }}}^{T} c_{p} d T .
\end{gathered}
$$

Die Berechnung der Wärmeleitfähigkeit in der porösen Hülle ist in Kap. 3.4 beschrieben. An der Phasengrenze zwischen der sich bildenden festen Phase und der noch nicht verdampften flüssigen Phase gilt, daß der Wärmefluß in der festen Phase gleich dem in der flüssigen Phase ist, zuzüglich der zur Verdunstung aufgebrachten Energie und der Energie der Partikel, welche 
sich an die feste Hülle anlagern:

$$
\begin{gathered}
\left.\lambda \frac{\partial T}{\partial r}\right|_{r=r_{\text {Phasengrenze }}}=\left.\lambda \frac{\partial T}{\partial r}\right|_{r=r_{\text {Phasengrenze }}} \\
-\dot{m} \cdot h_{v}-\dot{m}_{\text {Dampf }} \cdot c_{p_{\text {Dampf }}} \cdot T-\dot{m}_{\text {Partikel }} \cdot c_{p_{\text {Partikel }}} \cdot T
\end{gathered}
$$

Der Dampfmassenstrom berechnet sich wegen des zusätzlichen Stofftransportwiderstandes der Hülle aus

$$
\dot{m}=\left(\frac{1}{\beta}+\frac{\mu\left(r_{a}-r_{P g}\right)}{D}\right)^{-1} \frac{p_{O}-p_{U}}{R_{D} T} .
$$

Durch den zweite Term in der Klammer wird der Widerstand der Hülle berücksichtigt, wobei $D$ der Diffusionskoeffizient des Dampfes und $\mu$ der Diffusionswiderstandsfaktor ist. Die Differenz $\left(r_{a}-r_{P g}\right)$ bezeichnet die Dicke der Schale. $\beta$ ist der Stoffübergangskoeffizient.

\section{Feststoffbildung}

In der äußeren Schale wird eine weitere Populationsbilanz gelöst (Gl. 5.35). Da in der äußeren Schale keine Flüssigkeit und somit keine gelöste Substanz vorhanden ist, findet kein weiteres Wachstum der Partikel statt. Die Populationsbilanz des innersten Punktes der Hülle (Gl. 5.36) enthält zusätzlich noch einen Quell-Term, der die Anlagerung der Partikel aufgrund des sich zurückziehenden Trocknungsspiegels berücksichtigt. Da die Hülle wächst, wird durch den zweiten Term die Pseudo-Konvektion berücksichtigt:

$$
\begin{gathered}
\frac{\partial n}{\partial t}=\underbrace{\frac{\partial n}{\partial \tilde{r}} \frac{1}{r_{a}-r_{i}}\left[\frac{d r_{i}}{d t}+\tilde{r}\left(\frac{d r_{a}}{d t}-\frac{d r_{i}}{d t}\right)\right]}_{\text {"Pseudo-Konvektion" }} \\
\frac{\partial n}{\partial t}=\underbrace{\frac{\partial\left(n_{\text {Anlagerung }}\right)}{\partial t}}_{\text {Anlagerung }} \underbrace{+\frac{\partial n}{\partial \tilde{r}} \frac{1}{r_{a}-r_{i}}\left[\frac{d r_{i}}{d t}+\tilde{r}\left(\frac{d r_{a}}{d t}-\frac{d r_{i}}{d t}\right)\right]}_{\text {"Pseudo-Konvektion" }} .
\end{gathered}
$$

Zur Beschreibung der Anlagerung der Partikel aus der flüssigen Phase an der festen Hülle enthält die Populationsbilanz der flüssigen Phase in der äußersten flüssigen Schale einen Senkenterm:

$$
\frac{\partial n}{\partial t}=\underbrace{-\frac{\partial\left(n_{\text {Anlagerung }}\right)}{\partial t}}_{\text {Anlagerung }} \underbrace{\frac{\partial(G n)}{\partial L}}_{\text {Wachstum }} \underbrace{+\frac{\partial n}{\partial \tilde{r}} \frac{1}{r_{a}-r_{i}}\left[\frac{d r_{i}}{d t}+\tilde{r}\left(\frac{d r_{a}}{d t}-\frac{d r_{i}}{d t}\right)\right]}_{\text {"Pseudo-Konvektion" }} .
$$

Zur Bestimmung des Quellterms wird die Annahme getroffen, daß das Verhältnis von Partikelmasse zu Flüssigkeitsmasse gleich dem Verhältnis von Dampfmassenstrom zu Partikelmassenstrom ist.

$$
\frac{\dot{m}_{\text {Dampf }}}{\dot{m}_{\text {Partikel }}}=\frac{m_{\text {Flüssigkeit }}}{m_{\text {Partikel }}}
$$




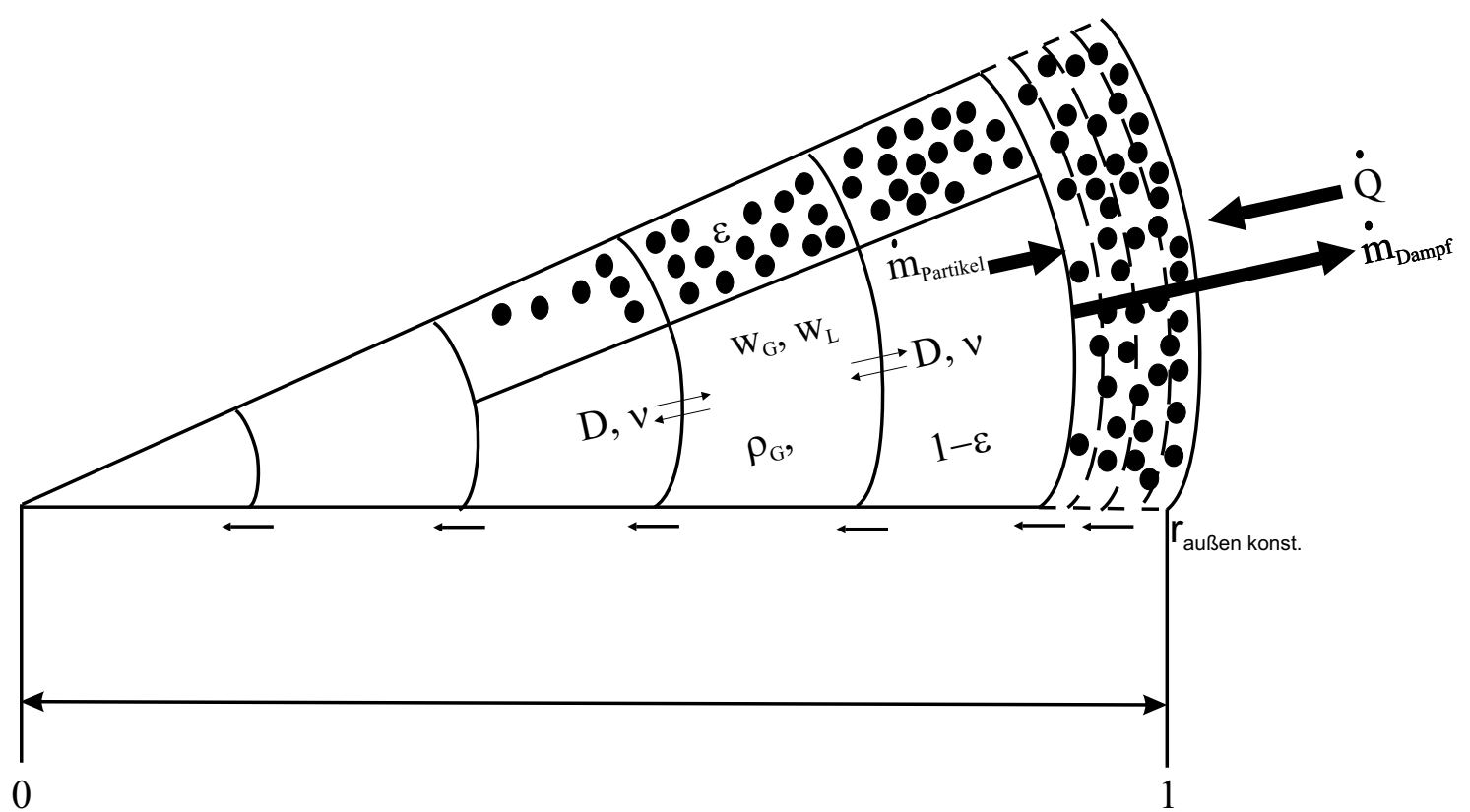

Abbildung 5.6: Mathematisches Modell des 2. Trocknungsabschnitts mit einem Koordinatensystem: Die Stützstellen, bei denen ein hoher Partikelvolumenanteil entstanden ist, werden als Hülle definiert und bewegen sich mit einer anderen Geschwindigkeit als die inneren, die den flüssigen Kern darstellen.

Als Partikelstrom ergibt sich daraus

$$
\left.\frac{\partial n(L)}{\partial t}\right|_{r=r_{P g}}=n\left(r_{\text {Phasengrenze }}, L\right) \cdot \frac{\dot{m}_{\text {Dampf }}}{m_{\text {Flüssigkeit }}} .
$$

Die Bewegung der Phasengrenze flüssig-fest, also das Wachsen der Hülle, berechnet sich dann aus

$$
\frac{d r}{d t}=\frac{\dot{m}_{\text {Dampf }}}{\rho_{\text {Dampf }}}+\frac{\dot{m}_{\text {Partikel }}}{\rho_{\text {Partikel }} \cdot 4 \pi r^{2}},
$$

wobei $\mu$ den Diffusionswiderstandsfaktor und $r_{a}$ und $r_{P g}$ den äußeren und inneren Radius der Feststoffhülle bezeichnen.

\subsubsection{Simulation des mehrschaligen Tropfens in einem Koordinatensystem}

Das mathematische Modell ist in Abb. 5.6 anschaulich dargestellt. Zur Simulation der Vorgänge muß der äußere Diskretisierungspunkt festgehalten werden, während die inneren Punkte sich entsprechend der Verschiebung der Phasengrenze fest/flüssig bewegen. Dadurch wird der Teil des Koordinatensystems, der den flüssigen Kern darstellt, weiter zusammengestaucht, während der Bereich, der die flüssige Hülle darstellt, gestreckt wird. Die Geschwindigkeit, mit 
der die Diskretisierungspunkte sich im äußeren Bereich bewegen, ist

$$
\frac{r_{a}-r}{r_{a}-r_{i}} \cdot \frac{d r_{\text {Schale }}}{d t}
$$

Im inneren Bereich haben die Diskretisierungspunkte die Geschwindigkeit

$$
\frac{r}{r_{a}} \cdot \frac{d r_{\text {Schale }}}{d t}
$$

Da die wichtigen Prozesse am Tropfenrand stattfinden, sind die Punkte im Koordinatensystem nicht äquidistant verteilt. Im äußeren Bereich werden mehr Punkte verwendet als im inneren.

Da die Verdunstung an der Phasengrenze flüssig/fest stattfindet, muß sowohl in der Gleichung für den Massen- als auch in der für den Energietransport an dieser Stelle eine Senke berücksichtigt werden. Der Dampfmassenstrom berechnet sich auch hier mit Gl. (5.34). Der Vorteil dieses Modellierungsweges besteht darin, daß keine Übergangsbedingung für die Partikel festgelegt werden muß, die sich aus der Flüssigkeit an die feste Hülle anlagern. Auch werden keine unterschiedlichen Gleichungen für die Energiebilanzen in der Flüssigkeit und der festen Hülle benötigt, wodurch auch die Problematik der Berechnung der Phasengrenztemperatur entfällt.

\subsubsection{Bildung einer Gasblase und Aufblähen des Tropfens}
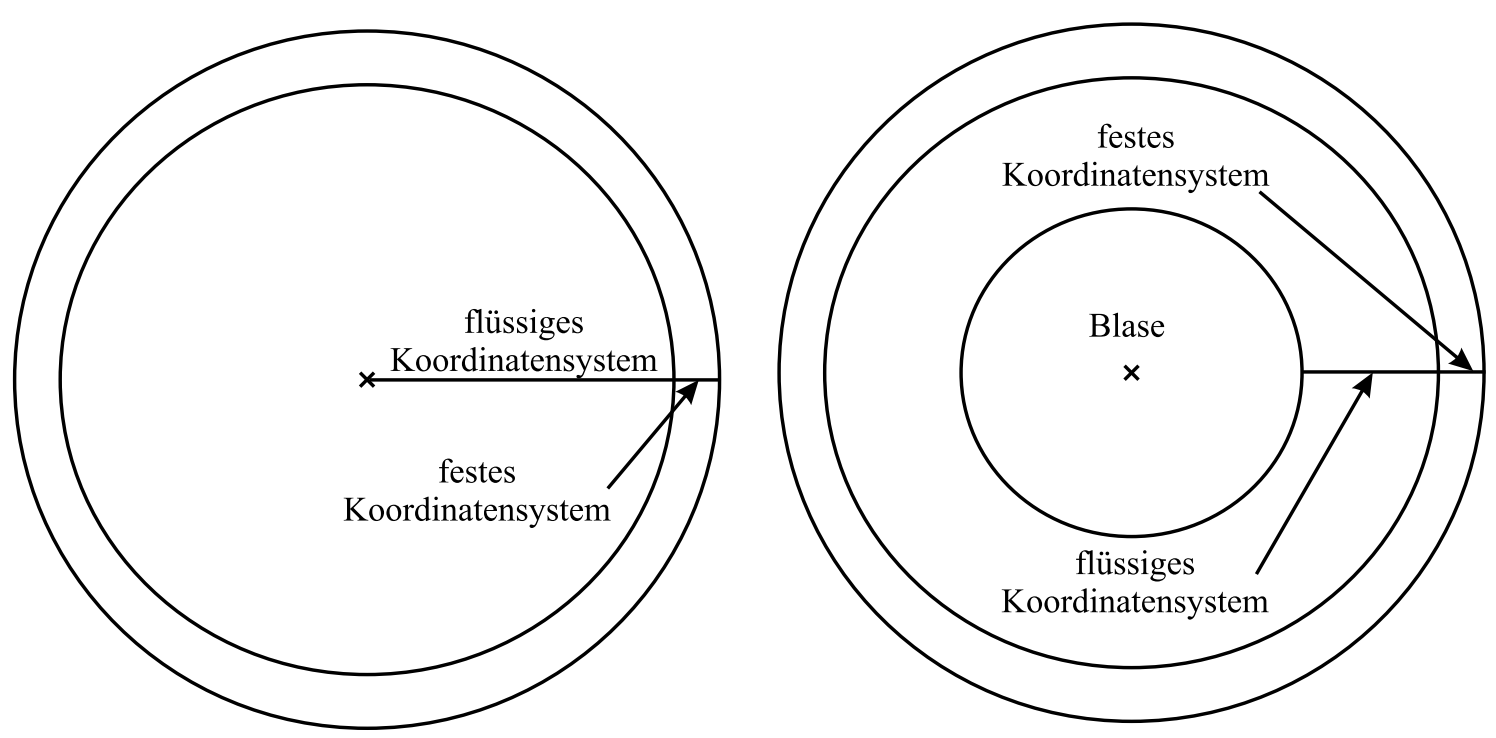

Abbildung 5.7: Das Koordinatensystem, welches den flüssigen Kern darstellt, schrumpft durch die Blasenbildung. Die Blase wird nicht durch ein eigenes Koordinatensystem dargestellt.

Wie bereits in Kapitel 3.2 beschrieben, gibt es zwei Möglichkeiten für die Blasenbildung im Inneren. Zum einen kann sich der Tropfen durch die Hüllenbildung auf die Siedetemperatur des Lösungsmittels aufheizen, wodurch sich im Tropfen Lösungsmitteldampf bildet. Zum 
anderen kann Trocknungsgas von außen durch Unterdruck in das Innere gelangen. Durch Kapillarkräfte wird die Flüssigkeit von innen durch die Poren der festen Hülle an die Oberfläche transportiert. Sind alle Poren mit Flüssigkeit gefüllt, entsteht durch die kapillaren Zugkräfte ein Unterdruck im Inneren. Fällt eine Pore trocken, dringt Luft von außen zum Druckausgleich ein. Zur Simulation des Prozesses muß der innere Punkt des Koordinatensystems, welches den flüssigen Kern abbildet, ebenfalls bewegt werden. Dieser Punkt bewegt sich nun entsprechend der Verschiebung der Phasengrenze gasförmig/flüssig im Tropfen nach außen, wodurch das innere Koordinatensystem gestaucht wird (Abb. 5.7). In der Blase treten keine Temperaturoder Konzentrationsgradienten auf. Daher wird zur Darstellung der Blase kein weiteres Koordinatensystem benötigt.

Bildet sich die Blase aus dem Lösungsmittel, so findet der Prozeß aufgrund von Verdampfung statt. Zur Bildung einer Blase kann es aber nur kommen, wenn die Verdampfung an der Tropfenoberfläche so stark behindert ist, daß im Tropfen die Siedetemperatur überschritten wird. Andernfalls würde die für die Verdampfung im Inneren notwendige Energie bereits an der Tropfenoberfläche aufgebraucht. Der Dampfmassenstrom an der Phasengrenze Flüssigkeit/Blase ergibt sich aus der durch Wärmeleitung an die Phasengrenze in der Flüssigkeit herantransportierten Wärme

$$
\dot{m}_{\text {Dampf }}=\left.\frac{1}{h_{v}} \lambda \frac{d T}{d r}\right|_{r=r_{P g}} .
$$

Die Veränderung des Radius der Blase berechnet sich aus

$$
\frac{d r}{d t}=\frac{\dot{m}_{D a m p f}}{\rho_{L}}
$$

wobei $\rho_{L}$ die Dichte des Lösungsmittels darstellt. Die Temperatur in der Blase entspricht der Flüssigkeitstemperatur an der Phasengrenze, d. h. der Siedetemperatur. Durch den Gasdruck werden Kräfte auf die feste Hülle ausgeübt, die sich dann je nach Stabilität aufbläht, was durch eine Bewegung der Koordinatensysteme für die Flüssigkeit und die Hülle simuliert werden kann. Der Druck in der Blase und somit der Druck auf die feste Hülle kann aus dem idealen Gasgesetz berechnet werden,

$$
p=\frac{m_{\text {Dampf }} R T}{V_{\text {Blase }}},
$$

mit der Dampfmasse $m_{\text {Dampf }}$ und dem Blasenvolumen $V_{\text {Blase }}$. Verdampfung und somit eine Veränderung des Blasenvolumens findet nur statt, wenn der Dampfdruck in der Blase geringer ist als der Dampfdruck an der Phasengrenze. Nimmt das Flüssigkeitsvolumen im Tropfen durch Verdampfung an der Tropfenoberfläche ab oder weitet sich die feste Hülle durch den Innendruck, so weitet sich die Blase auf, und es kann zu einer weiteren Verdampfung von Lösungsmittel in die Blase kommen. Steigt die Tropfentemperatur aufgrund der Behinderung der 
Verdampfung weiter, so steigt der Druck in der Blase gemäß dem idealen Gasgesetz und durch Verdampfung weiter. Die durch den Druck im Tropfeninneren entstehenden Zugspannungen in der Hülle können aus der Kesselformel berechnet werden [50]

$$
\sigma_{Z}=\frac{1}{2} p \frac{r}{s},
$$

wobei $p$ den Druck, $r$ den Innenradius der Hülle und $s$ die Dicke der Hülle bezeichnet. Da die Hülle eine Agglomeration von Einzelpartikeln darstellt, wird die maximale Zugspannung, die die Hülle erträgt, aus

$$
\sigma=\frac{\epsilon}{1-\epsilon} \frac{F}{\bar{x}_{1,2}^{2}}
$$

berechnet [51], wobei $\epsilon$ den Feststoffvolumenanteil, $\mathrm{F}$ die Haftkraft und $\bar{x}_{1,2}$ die mittlere Partikelgröße (Oberfläche-Volumen) bezeichnet. Die Gleichung leitet sich aus einer Kräftebilanz um ein Partikel her. Als Haftkraft wirken zwischen den Partikeln Flüssigkeitsbrücken, Van-der-Waals-Kräfte und elektrostatische Kräfte. Dabei sind die Haftkräfte, die durch Vander-Waals-Kräfte und Flüssigkeitsbrücken hervorgerufen werden, am größten. Auch können die Partikel durch Kristallisation zusammengewachsen sein. Die van-der-Waals-Kräfte werden bei gleich großen Kugeln mit

$$
F=\frac{A}{12 a_{p}^{2}} r
$$

berechnet [51]. A stellt die Hamaker-Konstante dar, $a_{p}$ den Partikelabstand und $r$ den Partikelradius.

Dringt Trocknungsgas von außen ein, so ergibt sich die Bewegung der Phasengrenze aus der Volumenänderung des flüssigen Kerns aufgrund der Verdunstung des Lösungsmittels an der Tropfenoberfläche. Da das Tropfenvolumen konstant bleibt, muß das verdunstende Flüssigkeitsvolumen durch Trocknungsgas von außen aufgefüllt werden. 


\section{Kapitel 6}

\section{Numerische Lösung der Differentialgleichungen}

Die Gleichungen, die die zeitliche Änderung der radialen Verteilung des Massenanteils der gelösten Substanz, der radialen Temperaturverteilung und die radiale Verteilung der Partikelanzahldichte beschreiben, müssen gekoppelt gelöst werden. Durch die Temperatur wird die Schrumpfungsgeschwindigkeit des Tropfens vorgegeben, von der wiederum die Verteilung der gelösten Substanz und damit die Kristallisation abhängen. Es handelt sich um ein System gekoppelter Differentialgleichungen unterschiedlichen Typs. Bei den Gleichungen zur Berechnung von Temperatur und Konzentration handelt es sich um parabolische Gleichungen. Populationsbilanzen hingegen gehören zum Typ der hyperbolischen Differentialgleichungen. Die hyperbolischen Differentialgleichungen enthalten im Gegensatz zu den parabolischen keinen dämpfenden Term. Während bei den Diffusionsvorgängen steile Gradienten flacher werden und sich allmählich ein gleichmäßiger Verlauf ausbildet, bleibt bei den Populationsbilanzen der steile Gradient bestehen. Diesen Eigenheiten haben die für die Lösung der Gleichungen einzusetzenden numerischen Verfahren Rechnung zu tragen. In den folgenden beiden Abschnitten werden die ausgewählten numerischen Verfahren erläutert.

\subsection{Lösung der Diffusionsgleichungen}

Zur Lösung der parabolischen Differentialgleichungen wird die vertikale Linienmethode verwendet. Die Differentialgleichungen werden semidiskretisiert. Die örtlichen Ableitungen werden durch Differenzenquotienten ersetzt, und die zeitlichen Ableitungen werden in ihrer kontinuierlichen Form belassen. Dadurch ergeben sich gewöhnliche Differentialgleichungen, die mit Standardlösern für gewöhnliche Differentialgleichungen gelöst werden können. Für die ersten 
örtlichen Ableitungen wird das Upwind-Verfahren erster Ordnung eingesetzt

$$
\frac{\partial u_{i}}{\partial x}=\frac{u_{j, i+1}-u_{j, i-1}}{h_{i}-h_{i-1}} \text {, wobei } h_{i}=x_{i+1}-x_{i}
$$

Die zweiten örtlichen Ableitungen werden nach dem Verfahren der zentralen Differenzen diskretisiert, wobei die Diskretisierung nach R. F. Sincovec und N. K. Madson erfolgt [52]. Im Intervallinneren gilt:

$$
\begin{gathered}
\frac{1}{x^{c}} \frac{\partial}{\partial x}\left(x^{c} D_{k, j}(t, x, u) \frac{\partial u_{j}(t, x)}{\partial x}\right)= \\
\frac{c+1}{\left(x_{i+1 / 2}\right)^{c+1}-\left(x_{i-1 / 2}\right)^{c+1}}\left[\left(x_{i+1 / 2}\right)^{c} D_{k, j, i+1 / 2}\left(\frac{u_{j, i+1}-u_{j, i}}{h_{i}}\right)-\right. \\
\left.\left(x_{i-1 / 2}\right)^{c} D_{k, j, i-1 / 2}\left(\frac{u_{j, i}-u_{j, i-1}}{h_{i-1}}\right)\right] \text { mit } \mathrm{j}=1,2, \ldots, \mathrm{n} .
\end{gathered}
$$

Für den linken und den rechten Rand des Systems haben die Gleichungen die folgende Form, wobei hier nur die Gleichung des linken Randes dargestellt ist:

$$
\begin{gathered}
\frac{1}{x^{c}} \frac{\partial}{\partial x}\left(x^{c} D_{k, j}(t, x, u) \frac{\partial u_{j}(t, x)}{\partial x}\right)= \\
\frac{c+1}{x_{1+1 / 2}^{c+1}-x_{1}^{c+1}}\left[\left(x_{1+1 / 2}\right)^{c} D_{k, j, 1}\left(\frac{u_{j, 2}-u_{j, 1}}{h_{1}}\right)-x_{1}^{c} D_{k, j, 1}\left(\frac{\gamma-\alpha_{j} u_{j, 1}}{\beta_{j}}\right)\right] .
\end{gathered}
$$

Der zweite Gradient in Gleichung (6.3) muß aus der Randbedingung mit $\gamma, \alpha$ und $\beta$ vorgegeben werden. $c$ kann die Werte 0,1 und 2 annehmen, je nachdem, ob in kartesischen, Zylinder- oder Kugelkoordinaten gerechnet wird. Es entstehen so gewöhnliche Differentialgleichungen, die mit einem Standardlösungsverfahren gelöst werden können.

\subsection{Lösung der Populationsbilanzen}

Die Populationsbilanzen müssen mit einem anderen numerischen Verfahren als die Diffusionsgleichungen gelöst werden, weil den Populationsbilanzen ein dämpfender Diffusionsterm fehlt. Bei Verwendung von zentralen Differenzen würde es zu starker numerischer Diffusion und Oszillationen an steilen Gradienten kommen. Zur Lösung des hyperbolischen Gleichungssystems steht eine Reihe von Verfahren zur Verfügung (Abb. 6.1).

Die Momenten-Methode ist in der Vergangenheit häufig zur Berechnung von Partikelgrößenverteilungen verwendet worden. Mit ihr lassen sich integrale Eigenschaften der Verteilung berechnen. Das k-te Moment einer Verteilung wird mit

$$
m_{k, r}=\int_{L_{\min }}^{L_{\max }} L^{k} n(L, t) d x
$$




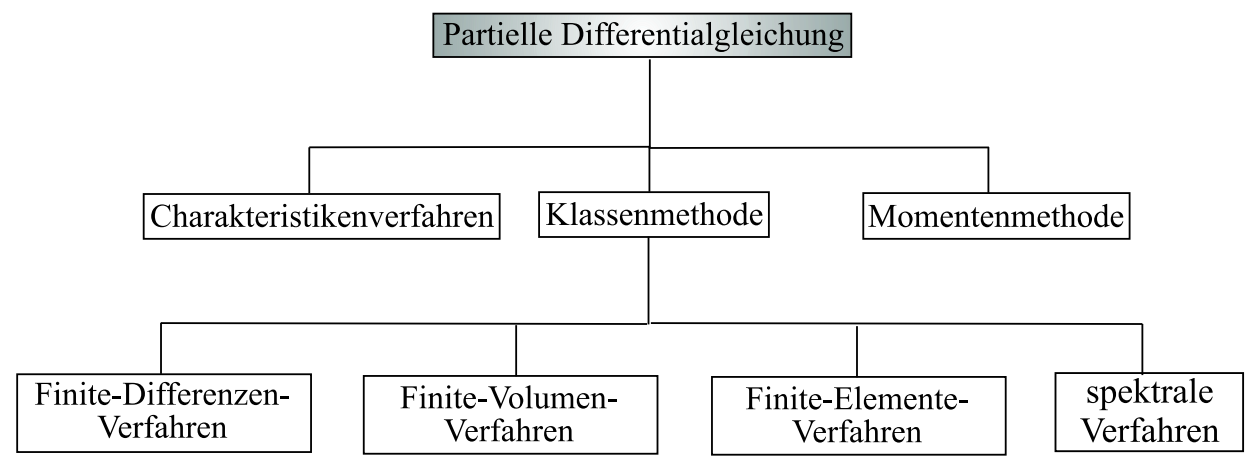

Abbildung 6.1: Verschiedene Lösungsverfahren für Populationsbilanzen (Erläuterung siehe nachfolgender Text)

berechnet. Durch Multiplikation der Populationsbilanz mit $L^{k}$ und Integration über die Partikelgröße ergibt sich ein System von Differentialgleichungen. Der Vorteil der MomentenMethode besteht in der geringen Zahl an Differentialgleichungen, die gelöst werden müssen. Die Methode kann jedoch nur verwendet werden, wenn der Typ der Verteilung bekannt ist, weil verschiedene Verteilungen die gleichen Momente haben können. Auch ist eine Schließbedingung notwendig, die das Gleichungssystem begrenzt [53].

Bei dem Charakteristikenverfahren wird die Differentialgleichung in ein System gewöhnlicher Differentialgleichungen transformiert, welches entlang der Partikelpfade (Charakteristiken) gelöst wird. Das Koordinatensystem bewegt sich mit der Partikelgeschwindigkeit. Beim Charakteristikenverfahren machen sich nur Diffusion, Reaktion und die rechte Seite der Differentialgleichung bemerkbar, nicht jedoch die Konvektion steiler Fronten. Daher erlaubt diese Methode große Zeitschritte. Da die Differentialgleichungen beim Charakteristikenverfahren in einem Lagrange-Koordinatensystem transformiert werden, treten jedoch sehr oft zusätzliche analytische und numerische Probleme auf [54].

Bei der Klassenmethode wird der physikalische Raum in Klassen unterteilt, so daß sich für jede Klasse eine Differentialgleichung ergibt. Für die Klassenmethode gibt es verschiedene Diskretisierungsverfahren.

Finite-Volumen-Verfahren: Die Partikelanzahldichte wird in diskreten Volumen berechnet, wobei der Funktionswert im Mittelpunkt des betrachteten Volumens berechnet wird. Die zeitliche Änderung der Anzahldichte im Volumenelement ergibt sich aus der Differenz der über die Ränder zu- und abfließenden Ströme, wobei die Ränder mit n(orth), s(outh), e(ast) und $\mathrm{w}$ (est) bezeichnet werden [55]:

$$
V_{i} \frac{n_{i}^{t+1}-n_{i}^{t}}{\Delta t}=\left(\left.\Phi_{x}\right|_{e}-\left.\Phi_{x}\right|_{w}\right) \Delta y+\left(\left.\Phi_{y}\right|_{n}-\left.\Phi_{y}\right|_{s}\right) \Delta x
$$

Finite-Differenzen-Verfahren: Bei den Finite-Differenzen-Verfahren wird die Partikelanzahldichte an diskreten Punkten berechnet. Der Übergang zwischen Finite-Differenzen- und 
Finite-Volumen-Verfahren ist fließend, weil durch Division der rechten Seite durch das Volumen finite Volumen in finite Differenzen übergehen.

Spektrale Verfahren: Bei diesen Verfahren wird die Gleichung zwischen zwei Diskretisierungspunkten durch bekannte glatte Funktionen angenähert. Die einzelnen Funktionen werden zu einer den gesamten physikalischen Raum umfassenden Gleichung zusammengefaßt und integriert. Zu den spektralen Verfahren gehören die Kollokations-, Galerkin- und Tau-Verfahren [56].

Finite-Elemente-Verfahren: Bei diesen Verfahren wird die Gleichung ebenfalls zwischen zwei Diskretisierungspunkten durch bekannte glatte Funktionen angenähert. Die einzelnen Funktionen werden zu einer den gesamten physikalischen Raum umfassenden Gleichung zusammengefasst und integriert. Im Gegensatz zu den spektralen Verfahren sind die Funktionen jedoch nur für ein finites Element definiert [57].

Der Vorteil der Klassenmethoden besteht darin, daß der gesamte Verlauf der Verteilung berechnet wird, der Nachteil besteht in der großen Anzahl zu lösender Gleichungen.

\subsubsection{Verwendete Diskretisierungsmethode}

In dieser Arbeit ist die Klassenmethode verwendet worden, weil mit dieser Methode keine Information über die Partikelstruktur verloren geht und sie mathematisch relativ einfach umzusetzen ist. Das eingesetzte Verfahren ist ein Slope-Limiter-Verfahren von Pennigton und Berzins [58]. Es ist ebenfalls semidiskret, wodurch eine Kopplung mit dem zentralen Differenzen-Verfahren einfach möglich ist. Bei dem Verfahren handelt es sich um ein FiniteVolumen-Verfahren, welches die zeitliche Änderung des Funktionswertes aus den Flüssen über die Ränder eines Kontrollvolumens berechnet. Die Werte an den Rändern eines Kontrollvolumens werden je nach Richtung des Informationsflusses jeweils aus den beiden vorhergehenden Werten von rechts bzw. links extrapoliert (Abb. 6.2). Im Bereich glatter Kurvenverläufe ist das Verfahren zweiter Ordnung und an Unstetigkeiten erster Ordnung. Eine Limiter-Funktion $\Theta$ entscheidet durch einen Vergleich der Steigungen links und rechts des betrachteten Punktes, ob zweiter oder erster Ordnung gerechnet werden muß,

$$
\begin{array}{r}
n_{k+1 / 2}^{L}=n_{k}+\frac{L_{k+1}-L_{k}}{2} \cdot \frac{n_{k}-n_{k-1}}{L_{k}-L_{k-1}} \cdot \Theta\left(\frac{\Delta n_{k+1}}{\Delta n_{k}}\right) \\
n_{k+1 / 2}^{R}=n_{k+1}+\frac{L_{k+1}-L_{k}}{2} \cdot \frac{n_{k+2}-n_{k+1}}{L_{k+2}-L_{k+1}} \cdot \Theta\left(\frac{\Delta n_{k+1}}{\Delta n_{k+2}}\right)
\end{array}
$$

mit

$$
\Delta n_{k}=\frac{n_{k}-n_{k-1}}{L_{k}-L_{k-1}} .
$$

$n^{R}$ und $n^{L}$ stehen für die rechts- bzw. linksseitig angenäherten Funktionswerte an den Rändern des Kontrollvolumens und L kennzeichnet die Diskretisierung. Die numerische Flußfunktion 

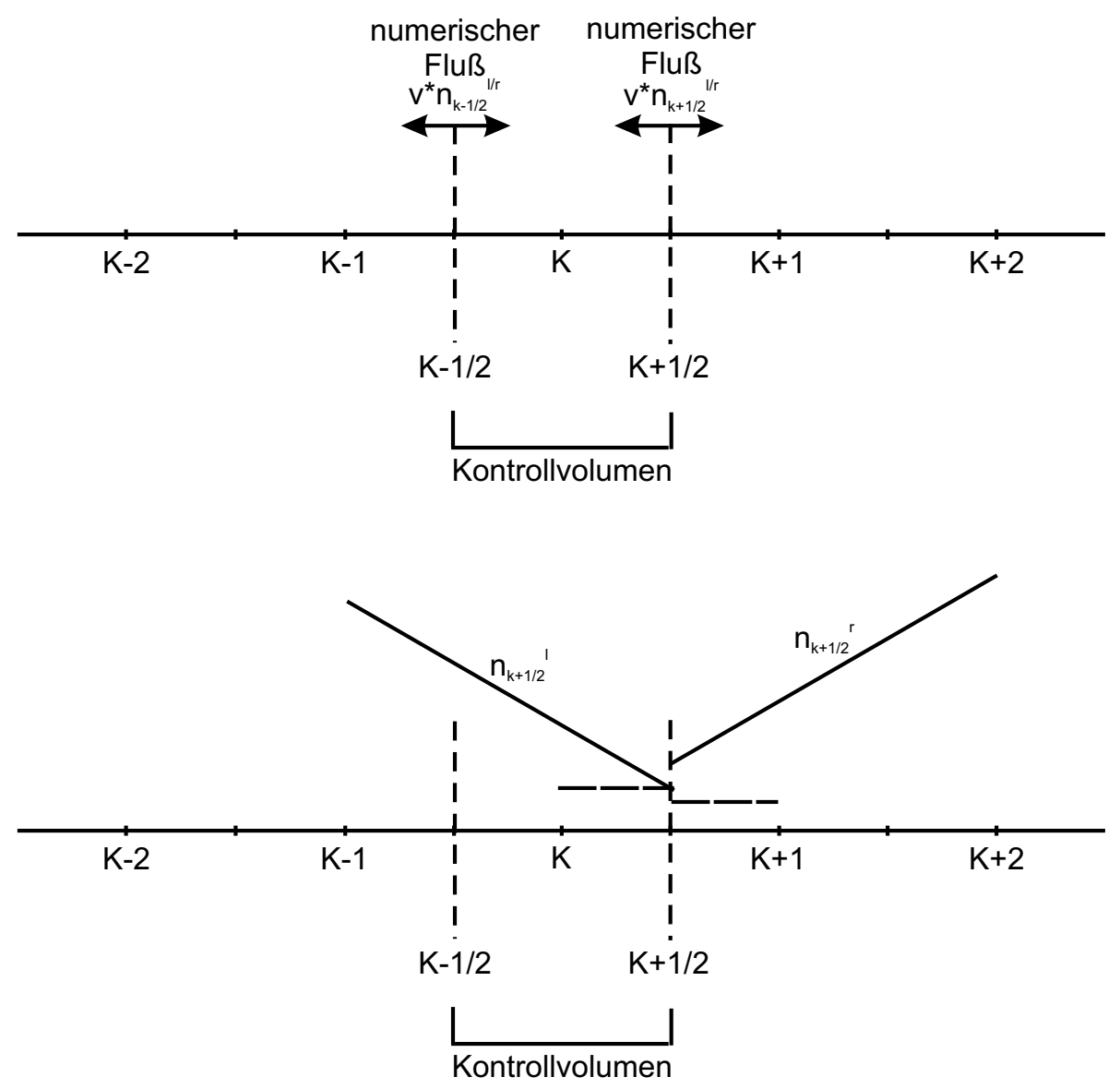

Abbildung 6.2: Oben: Veranschaulichung der Diskretisierung nach Pennigton und Berzins [58]: $\mathrm{n}_{k \pm 1 / 2}$ sind die Funktionswert an den Rändern des Kontrollvolumens und v die jeweilige Flußgeschwindigkeit; unten: Berechnung der Funktionswerte an den Kontrollvolumenrändern 


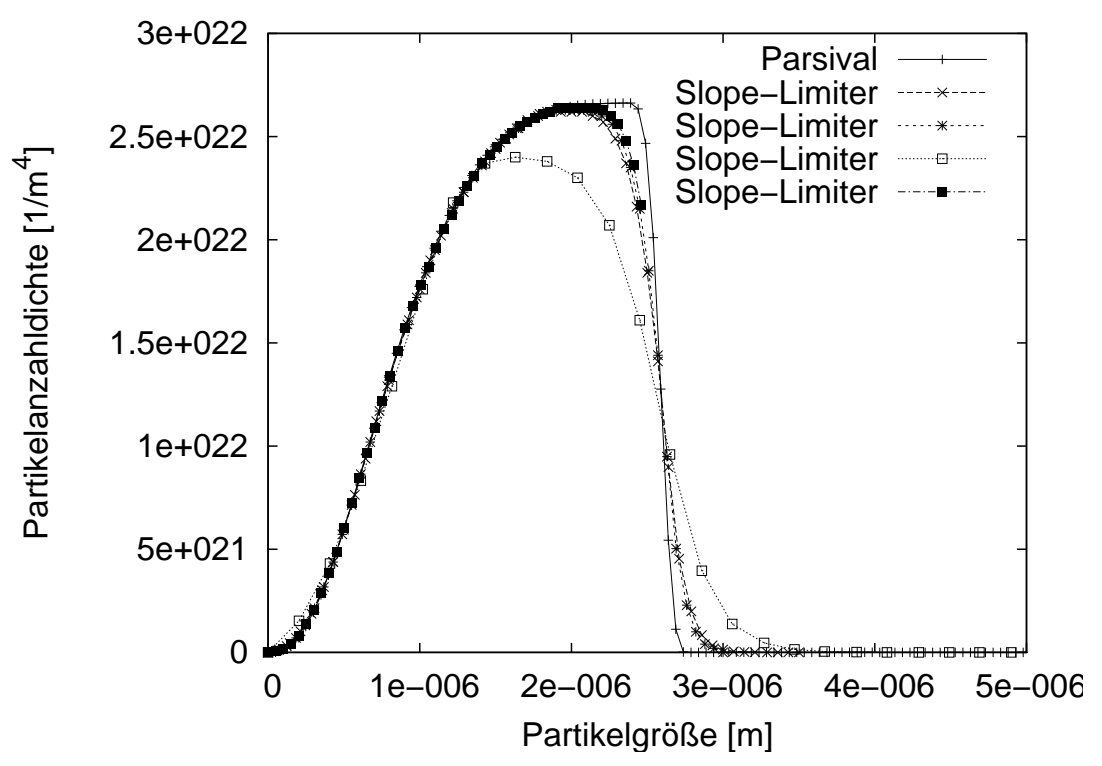

Abbildung 6.3: Vergleich der numerischen Diffusion bei dem von Parsival ${ }^{\circledR}$ verwendeten Lösungsverfahrens mit der numerischen Diffusion beim Slope-Limiter-Verfahren bei einer unterschiedlichen Zahl an Stützstellen

ergibt sich dann aus der Wachstumsgeschwindigkeit und dem Funktionswert

$$
\begin{gathered}
\hat{f}_{k+1 / 2}=G\left(\frac{L_{k}+L_{k+1}}{2}\right) \cdot n_{k+1 / 2}^{L} \text {, falls } G\left(\frac{L_{k}+L_{k+1}}{2}\right)>0 \\
\text { bzw. } \hat{f}_{k+1 / 2}=G\left(\frac{L_{k}+L_{k+1}}{2}\right) \cdot n_{k+1 / 2}^{R} \text {, falls } G\left(\frac{L_{k}+L_{k+1}}{2}\right)<0 .
\end{gathered}
$$

Aus den Flußfunktionen wird die Ableitung nach der Partikellänge bzw. dem Ort diskretisiert. Die zeitliche Ableitung wird in ihrer kontinuierlichen Form belassen. Hier liegt der Vorteil dieses Verfahrens, weil es mit einem klassischen Standardlösungsverfahren gelöst werden kann. Die Ortsableitung ergibt sich dann gemäß

$$
\left.\frac{\partial(G n)}{\partial L}\right|_{L=L_{k}} \approx \frac{\hat{f}_{k+1 / 2}-\hat{f}_{k-1 / 2}}{1 / 2 \cdot\left(L_{k+1}-l_{k-1}\right)}
$$

Die Güte eines numerischen Verfahrens zur Lösung hyperbolischer Differentialgleichungen läßst sich unter anderem an der Größe der numerischen Diffusion ablesen. Ein weiteres Kriterium ist das Auftreten von Schwingungen an steilen Gradienten. Das verwendete Verfahren hat bei einer ausreichenden Zahl an Stützstellen relativ wenig numerische Diffusion und neigt nicht zum Oszillieren, weil es an Sprungstellen erster Ordnung rechnet. Abb. 6.3 zeigt einen Vergleich mit der kommerziellen Software Parsival ${ }^{\circledR}$, welche zur Lösung der Populationsbilanzen das h-p-Galerkin-Verfahren [59] verwendet. Es ist zu erkennen, daß bei einer ausreichenden Zahl an Stützstellen mit dem Slope-Limiter-Verfahren ähnlich steile Gradienten 


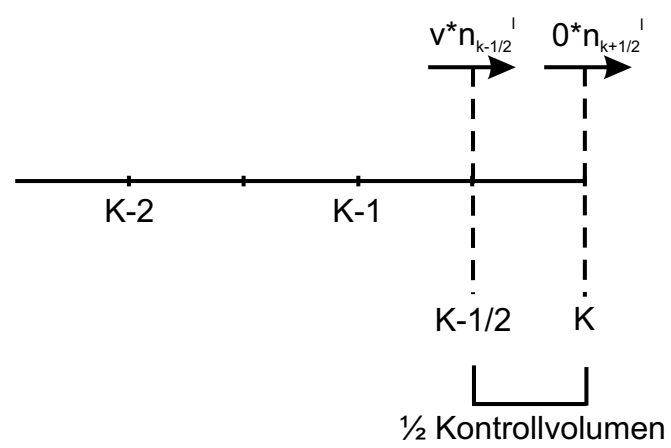

Abbildung 6.4: Anwendung des Verfahrens von Pennington und Berzins [58] am Tropfenrand (Bedeutung der Symbole wie in Abb. 6.2)

berechnet werden können wie mit Parsival ${ }^{\circledR}$. Zur Simulation der Kristallisation im Tropfen ist Parsival ${ }^{\circledR}$ jedoch nicht geeignet, weil nur ideal durchmischte Kristallisatoren berechnet werden können. Eine räumliche Beschreibung ist nur durch Hintereinanderschalten mehrerer Kristallisatoren möglich, wobei Diffusions- und Konvektionsströme durch Stoffströme zwischen den Kristallisatoren dargestellt werden müßten. Die Simulation der zeitlichen Änderung des Tropfenvolumens und die damit verbundene Bewegung der Phasengrenze ist jedoch nur durch zusätzliche algebraische Gleichungen möglich, die den Ort der Phasengrenze beschreiben.

\subsubsection{Randbedingung am äußeren Tropfenrand}

Im Falle des schrumpfenden Tropfens dürfen keine Partikel den Tropfen verlassen. Um dies zu erreichen, wird der numerische Fluß am äußeren Rand zu Null gesetzt. Dadurch akkumulieren die Partikel in der äußeren Zelle, weil nur Partikel aus dem Tropfeninneren hineinströmen können, aber keine hinaus. In der äußersten Zelle muß darüber hinaus berücksichtigt werden, daß das Kontrollvolumen nur halb so groß ist wie das der Zellen im Inneren (Abb. 6.4).

\subsection{Wahl des Lösers}

Zur Berechnung des Zeitschritts im 1. Trocknungsabschnitt und bei der Berechnung des 2 . Trocknungsabschnitts in einem Koordinatensystem kann ein explizites Runge-Kutta-Verfahren verwendet werden $[60,61]$. Bei der Berechnung des 2. Trocknungsabschnitts mit zwei Koordinatensystemen wird das Gleichungssystem jedoch sehr steif, so daß ein implizites Verfahren notwendig ist. Implizite Löser benötigen die Jakobi-Matrix zur Lösung des Differentialgleichungssystems, welche die Anzahl der Variablen zum Quadrat Eintragungen hat. Durch die Populationsbilanzen kommt eine weitere Dimension hinzu, weil es für jeden radialen Diskretisierungspunkt eine Anzahl von Partikelklassen gibt. Das bedeutet eine starke Vergrößerung des 


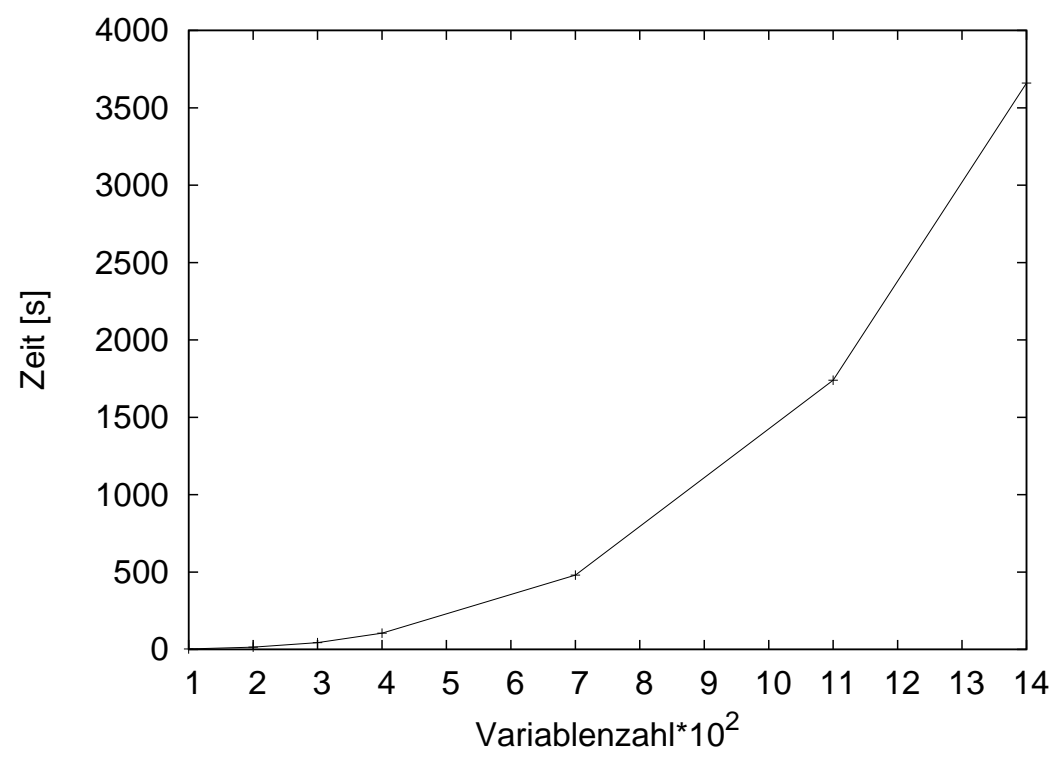

Abbildung 6.5: Zunahme der Rechenzeit mit zunehmender Variablenzahl

Variablensystems, und daher ist zur Lösung des Gleichungssystems mit einem impliziten Löser viel Speicherplatz notwendig. Der ausgewählte Löser verwendet das Rosenbrock-Verfahren [62]. In Abbildung 6.5 ist die Zunahme der Rechenzeit mit zunehmender Variablenzahl dargestellt. Es ist zu erkennen, daß die Rechenzeit proportional zur dritten Potenz der Variablenzahl zunimmt.

\subsection{Numerische Fehler}

Abbildung 6.6 zeigt den Einfluß der Pseudokonvektion. Der Peak bleibt am physikalischen Ort stehen, während das Koordinatensystem aufgrund der Verdampfung schrumpft. Durch die auf das Diskretisierungsverfahren zurückzuführende numerische Diffusion wird der Peak etwas breiter, und die Flanken werden flacher. Das unterste Diagramm in Abb. 6.6 zeigt den durch die Pseudokonvektion verursachten Fehler in der Partikelmassenbilanz, der jedoch relativ gering ist im Vergleich zur Unsicherheit der Kristallisationskinetiken, die im folgenden Kapitel erläutert werden. 

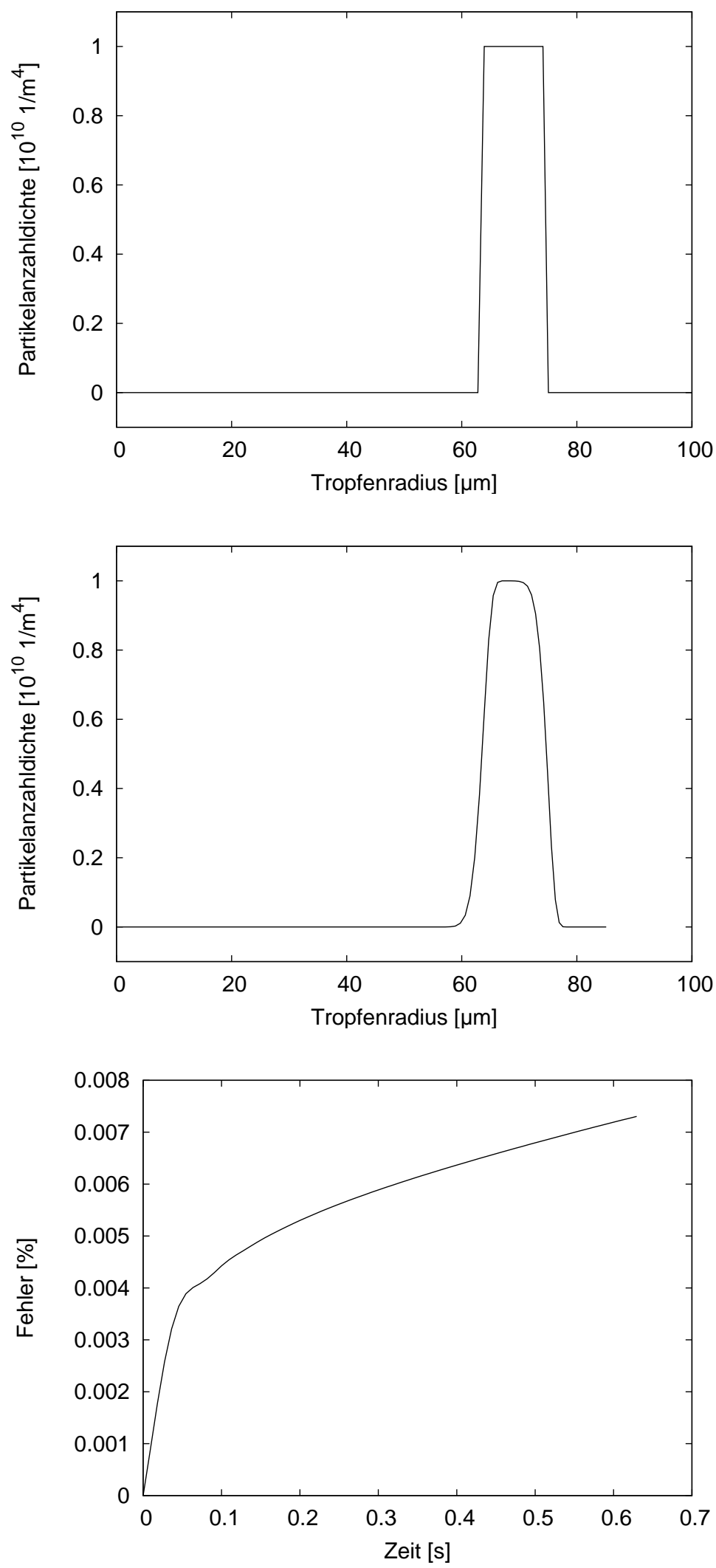

Abbildung 6.6: Oben: Partikelanzahldichteverteilung zum Zeitpunkt 0; Mitte: Partikelanzahldichteverteilung nach $0,63 \mathrm{~s}$; unten: Fehler in der integrierten Verteilungsfunktion durch die Pseudokonvektion 


\subsection{Numerik bei zwei Koordinatensystemen}

Um ein neues Koordinatensystem hinzufügen zu können, muß der Löser angehalten werden, weil während der Laufzeit die Anzahl der Variablen, die dem Löser übergeben wird, nicht geändert werden kann. Das neue Koordinatensystem, welches außen angefügt wird, ist so breit wie der Abstand zweier Diskretisierungspunkte im Koordinatensystem, das den flüssigen Kern beschreibt.

Die Partikel des äußeren Diskretisierungspunktes des Koordinatensystems, das den flüssigen Kern beschreibt, werden auf die Diskretisierungspunkte des neuen Koordinatensystems verteilt, so daßs sich die Anzahldichte nicht verändert. In Abbildung 6.7 ist dies exemplarisch dargestellt. Der äußere Diskretisierungspunkt des zweiten Koordinatensystems liegt an dem

\section{Ende des 1. Trocknungsabschnitts}

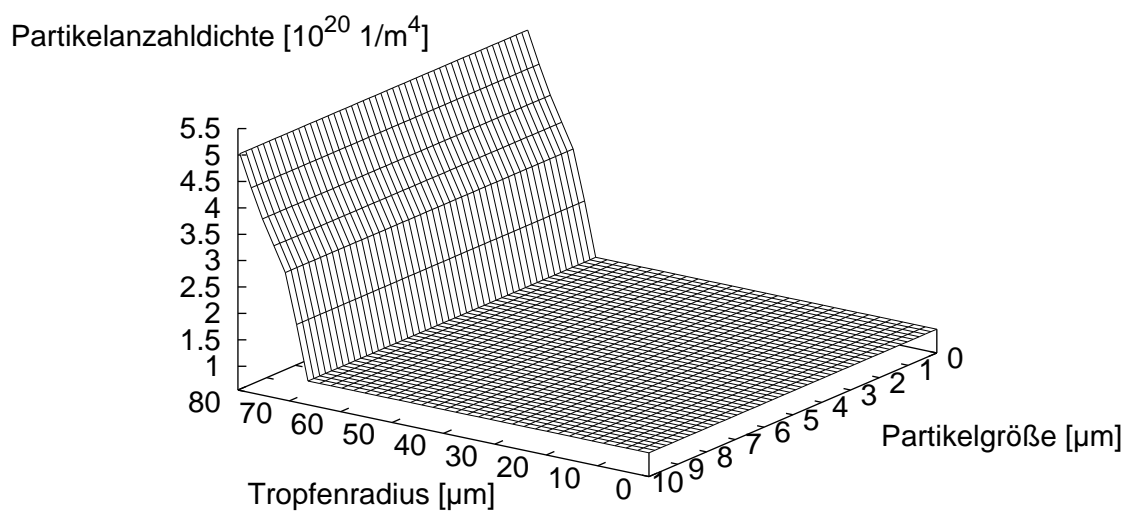

Beginn des 2. Trocknungsabschnitts

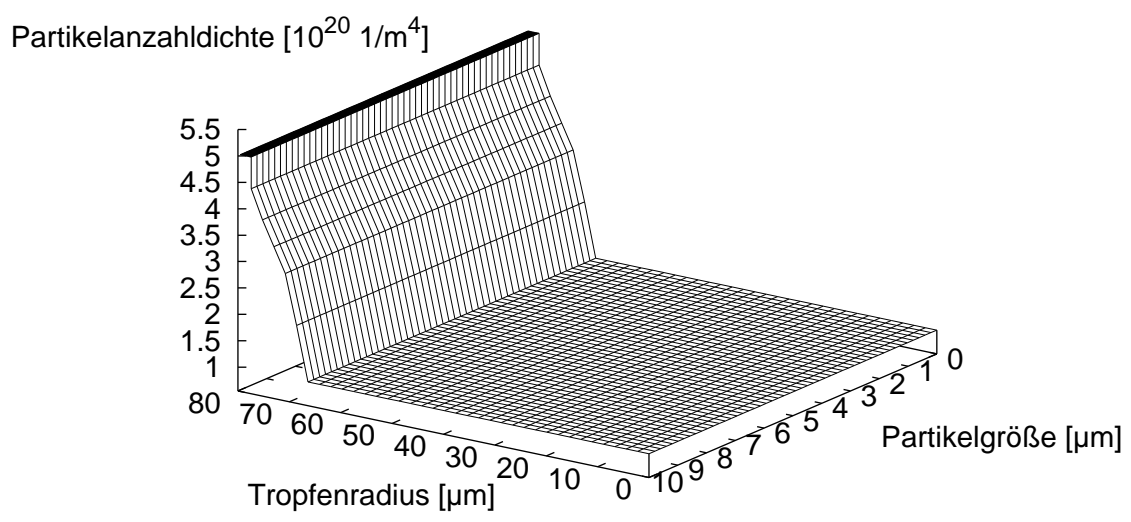

Abbildung 6.7: Die Information des äußeren Diskretisierungspunktes wird auf die Diskretisierungspunkte des zweiten Koordinatensystems verteilt 


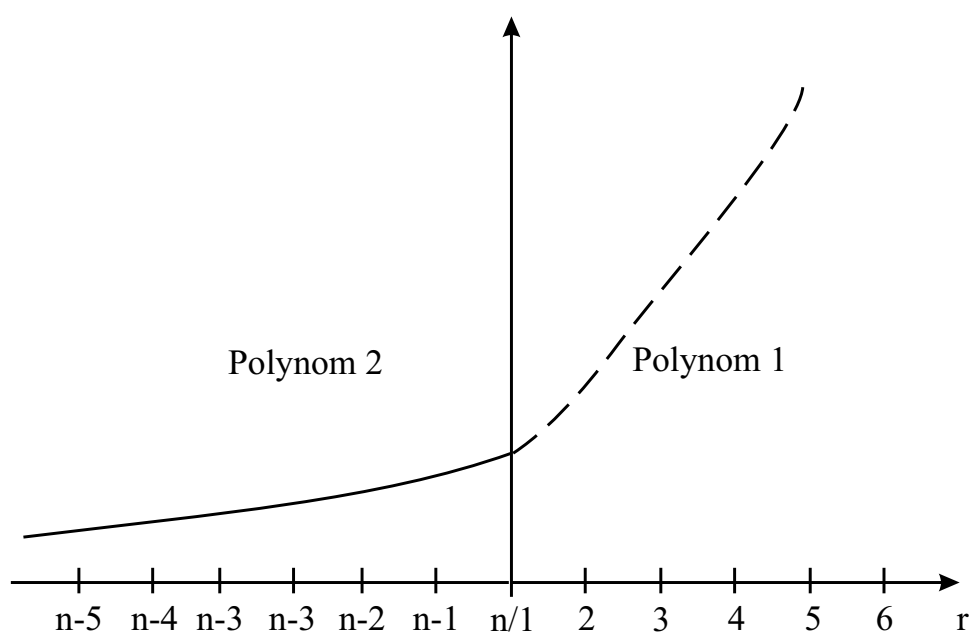

Abbildung 6.8: Die Phasengrenztemperatur wird durch je ein Lagrange-Polynom rechts und links der Phasengrenze berechnet.

selben Ort wie der äußere Punkt des Koordinatensystems, das den flüssigen Kern beschreibt, am Ende des 1. Trocknungsabschnitts.

\subsubsection{Berechnung des Temperaturverlaufs}

Zur Berechnung der Temperatur an der Phasengrenze fest/flüssig wird der Temperaturverlauf rechts und links der Phasengrenze durch je ein Lagrange-Polynom angenähert (Abb. 6.8) [63]. Das Lagrange-Polynom hat die allgemeine Form:

$$
\begin{gathered}
T(r)=\frac{\left(r-r_{2}\right)\left(r-r_{3}\right) \ldots\left(r-r_{N}\right)}{\left(r_{1}-T_{2}\right)\left(r_{1}-r_{3}\right) \ldots\left(r_{1}-r_{N}\right)} T_{1}+ \\
\frac{\left(r-r_{1}\right)\left(r-r_{2}\right) \ldots\left(r-r_{N}\right)}{\left(r_{2}-r_{1}\right)\left(r_{2}-r_{3}\right) \ldots\left(r_{2}-r_{N}\right)} T_{2}+\cdots+ \\
\quad \frac{\left(r-r_{1}\right)\left(r-r_{2}\right) \ldots\left(r-r_{N-1}\right)}{\left(r_{N}-r_{1}\right)\left(r_{N}-r_{3}\right) \ldots\left(r_{N}-r_{N-1}\right)} T_{N} .
\end{gathered}
$$

Die Polynome werden abgeleitet zu

$$
\begin{gathered}
\frac{d T_{1}}{d r}\left(r_{1}\right)=\frac{\left(r_{1}-r_{2}\right)+\left(r_{1}-r_{3}\right)}{\left(r_{1}-r_{2}\right)\left(r_{1}-r_{3}\right)} T_{1}+\frac{\left(r_{1}-r_{3}\right)}{\left(r_{2}-r_{1}\right)\left(r_{2}-r_{3}\right)} T_{2}+ \\
\frac{\left(r_{1}-r_{2}\right)}{\left(r_{3}-r_{1}\right)\left(r_{3}-r_{2}\right)} T_{3} \\
\frac{d T_{2}}{d r}\left(r_{1}\right)=\frac{\left(r_{n}-r_{n-1}\right)}{\left(r_{n-2}-r_{n-1}\right)\left(r_{n-2}-r_{n}\right)} T_{n-2}+\frac{\left(r_{n}-r_{n-2}\right)}{\left(r_{n-1}-r_{n-2}\right)\left(r_{n-2}-r_{n-1}\right)} T_{n-1}+ \\
\frac{\left(r_{n}-r_{n-2}\right)+\left(r_{n}-r_{n-1}\right)}{\left(r_{n}-r_{n-2}\right)\left(r_{n}-r_{n-2}\right)} T_{n}-\dot{m} \cdot h_{v}-\dot{m}_{\text {Partikel }} \cdot c_{p_{\text {Partikel }}} \cdot T,
\end{gathered}
$$


und die unbekannte Phasengrenztemperatur $T_{1}=T_{n}=T_{\text {Phasengrenze }}$ ergibt sich dann aus der Gleichung

$$
\lambda_{1} \frac{d T_{1}}{d r}\left(r_{1}\right)=\lambda_{2} \frac{d T_{2}}{d r}\left(r_{1}\right)-\dot{m} \cdot h_{v}-\dot{m}_{\text {Partikel }} \cdot c_{p_{\text {Partikel }}} \cdot T .
$$




\section{Kapitel 7}

\section{Experimentelles}

Zur experimentellen Untersuchung des Trocknungsprozesses in Einzeltropfen sind verschiedene Versuchsaufbauten denkbar. Die wichtigste Anforderung an den Versuch ist, daß die Anfangsund Randbedingungen reproduzierbar sind. Tabelle 7.1 zeigt bereits durchgeführte Einzeltropfenversuche verschiedener Autoren und den verwendeten Versuchsaufbau. Das Aufhängen des Tropfens an einem Draht bietet die Möglichkeit, den Trocknungsprozeß bei definierten Bedingungen ablaufen zu lassen und den Vorgang aufzeichnen zu können. Der Nachteil besteht darin, daß die Vorgänge im Tropfen durch den Draht beeinflußt werden und der Tropfen in der Regel größer als beim realen Prozeß im Sprühtrockner ist. In einer Reihe von Arbeiten ist der Trocknungsprozeß auf diese Art und Weise untersucht worden, wobei der Trocknungsprozeß mit Kameras aufgezeichnet worden ist [19, 38, 47, 64, 65]. Beim Tropfenlevitator kann der Prozeß ebenfalls gut beobachtet und aufgezeichnet werden. Darüber hinaus ist kein Draht vor-

Tabelle 7.1: Einzeltropfenversuche

\begin{tabular}{|l|l|}
\hline Autoren & Versuchsaufbau \\
\hline Charlesworth und Marshall (1960) [38] & Tropfen am Draht \\
Wahl (1974) [66] & fallender Tropfen \\
Büttiker (1981) [39] & fallender Tropfen \\
Cheong et al. (1986) [19] & Tropfen am Draht \\
Lee und Law (1991) [21] & fallender Tropfen \\
Tanigushi et al. (1999) [64] & Tropfen am Draht \\
Walton und Mumford (1999) [65] & Tropfen am Draht \\
Brenn et al. (2001) [27] & akustischer Tropfenlevitator \\
Yarin et al. (2002) [67] & akustischer Tropfenlevitator \\
Lin und Gentry (2003) [47] & Tropfen am Draht \\
\hline
\end{tabular}


handen, der den Prozeß beeinflussen könnte. Durch die Schallwellen werden jedoch Kräfte auf den Tropfen ausgeübt, die den Trocknungs- und Feststoffbildungsprozeß ebenfalls beeinflussen [68]. Brenn et al. [27] haben mit dem Ultraschalltropfenlevitator umfangreiche Untersuchungen zur Verdampfung und Feststoffbildung bei Einzeltropfen durchgeführt. Die Untersuchung eines Tropfens im freien Fall in einem Fallturm ist dem realen Geschehen im Sprühtrockner am Nächsten. Diese Untersuchungsmethode ist in den Arbeiten von Wahl [66], Büttiker [39] und Lee und Law [21] verwendet worden. Durch Tropfengeneratoren können Tropfen gleicher Größe erzeugt werden, so daß definierte Anfangsbedingungen möglich sind. Der Nachteil ist jedoch, daß der Trocknungsverlauf nicht ununterbrochen aufgezeichnet werden kann. Es kann nur die Partikelmorphologie auf verschiedenen Höhen und am Ende des Prozesses in Abhängigkeit von bestimmten Anfangs- und Randbedingungen untersucht werden.

\subsection{Durchgeführte Versuche}

In der vorliegenden Arbeit sind verschiedene Versuche zur Untersuchung des Trocknungsverlaufs gemacht worden. Zum einen sind Versuche in einem beheizten Fallrohr durchgeführt worden. Auch sind an einem Draht aufgehängte Tropfen untersucht worden.

\subsubsection{Versuche am Fallturm}

Das Fallrohr hat eine Länge von $10 \mathrm{~m}$ und einen Durchmesser von $100 \mathrm{~mm}$. Das Trocknungsgas kann mit einem Gaserhitzer auf Temperaturen von bis zu $200^{\circ} \mathrm{C}$ erhitzt werden. Zur Beheizung des Rohres steht darüber hinaus eine Begleitheizung über die Fallturmlänge zur Verfügung. Als Trocknungsgas können Luft oder Stickstoff verwendet werden.

Zur Erzeugung der Tropfen ist ein frequenzangeregter Tropfengenerator entwickelt worden (Abb. 7.1 und Abb. 7.2) [69]. Der Tropfengenerator besteht aus einer konisch geformten Kammer, deren oberen Abschluß ein Piezo-Summer bildet (Abb. 7.4), wobei dieser aus einer Metallscheibe besteht, auf deren Oberfläche ein Piezoquarz befestigt ist. Der Summer wird mit Hilfe des verstärkten Signals eines Frequenzgenerators in Schwingung versetzt, wobei für die Versuche ein Sinussignal verwendet wird. Der Strahl wird mit einer Kapillare oder Düse erzeugt, die an der Austrittsöffnung des Tropfengenerators mit einem Teflonstopfen befestigt bzw. eingeklebt wird (Abb. 7.3).

\section{Tropfenbildungsmechanismen}

Es werden verschiedene Tropfenbildungsmechanismen in Abhängigkeit von der Fließgeschwindigkeit der Flüssigkeit unterschieden (Abb. 7.5) [8] [5]: 


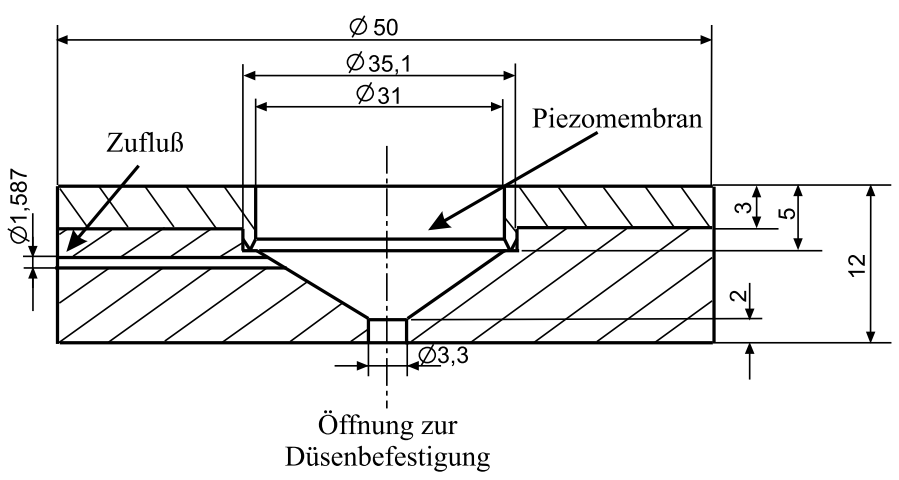

Abbildung 7.1: Aufbau des Tropfengenerators (Maße in $\mathrm{mm}$ )

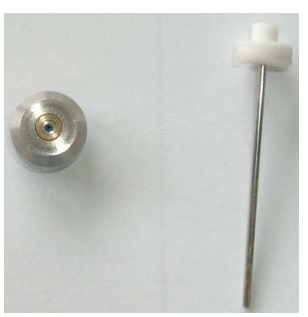

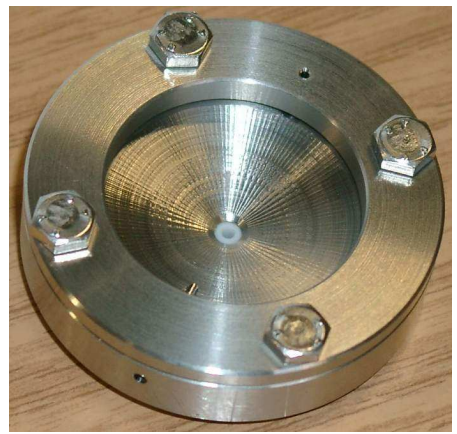

Abbildung 7.2: Gesamtansicht des Tropfengenerators

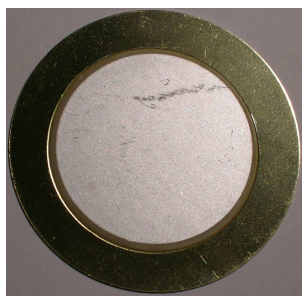

Abbildung 7.3: Links: Saphirdüse; rechts: Kapillare
Abbildung 7.4: Piezomembran (Durchmesser $35 \mathrm{~mm})$

1. Abtropfen: Die Flüssigkeit tropft ohne Strahlbildung von der Spitze der Kapillare ab, sobald die Gewichtskraft größer ist als die aus der Oberflächenspannung resultierenden Kräfte.

2. Rayleigh-Strahlzerfall: Der Flüssigkeitsstrahl zerfällt infolge von achsensymmetrischen Schwingungen an der Strahloberfläche, die von der Oberflächenspannung verursacht werden. Der Tropfendurchmesser ist größer als der Strahldurchmesser.

3. I. windinduzierter Strahlzerfall: Der Effekt der Oberflächenspannung wird durch die Relativgeschwindigkeit zwischen dem Strahl und dem umgebenden Gas verstärkt, wodurch sich der Zerfallsprozeß beschleunigt. Die Tropfen haben etwa den Durchmesser des Strahls.

4. II. windinduzierter Strahlzerfall: Die Tropfen werden durch das instabile Wachstum kurzwelliger Störungen an der Strahloberfläche erzeugt, die durch die Relativbewegung des Strahls im Gas entstehen. Dem Wachstum der Wellen wirkt die Oberflächenspannung entgegen. Der mittlere Tropfendurchmesser ist sehr viel kleiner als der Strahldurchmesser. 


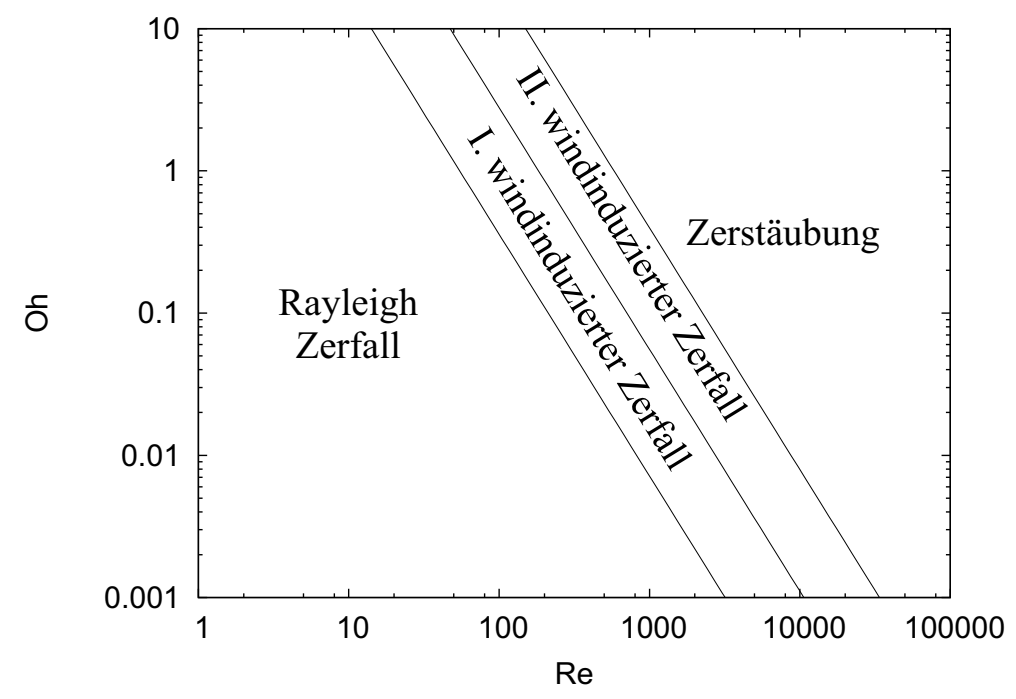

Abbildung 7.5: Einteilung der Zerfallsprozesse nach [5] (Oh: Ohnesorg-Zahl, Re: ReynoldsZahl)

5. Zerstäubung: Der Strahl zerreißt vollständig am Austritt. Der mittlere Tropfendurchmesser ist sehr viel kleiner als der Strahldurchmesser.

Zur Tropfenerzeugung wird in dieser Arbeit der Rayleigh-Strahlzerfall verwendet. In Abhängigkeit vom Strahldurchmesser und der Geschwindigkeit des Strahls läßt sich eine optimale Frequenz errechnen, bei der gleich große Tropfen entstehen. Die optimale Wellenlänge berechnet sich zu

$$
\begin{gathered}
\lambda_{o p t}=\pi d_{s} \sqrt{2+6 O h} \\
\text { mit der Ohnesorg-Zahl Oh= } \frac{\sqrt{W e}}{R e},
\end{gathered}
$$

wobei für niedrigviskose Flüssigkeiten $\lambda_{o p t}=4,4 d_{s}$ ist mit $d_{s}$ als dem Strahldurchmesser. $W e$ bezeichnet die Weber-Zahl, die in Kapitel 3.3 erläutert worden ist und Re die Reynolds-Zahl. Für den Tropfendurchmesser ergibt sich dann

$$
d_{\text {Tropfen }}=d_{s} \sqrt{44+6 O h},
$$

wobei für niedrigviskose Flüssigkeiten $d_{\text {Tropfen }}=1,9 d_{s}$ ist. Die Tropfenfrequenz, bei der monodisperse Tropfen entstehen, wird aus

$$
f_{o p t}=\frac{v}{\lambda_{o p t}}
$$

berechnet, wobei $v$ die Geschwindigkeit des Strahls am Zerfallsort ist [70][71]. 


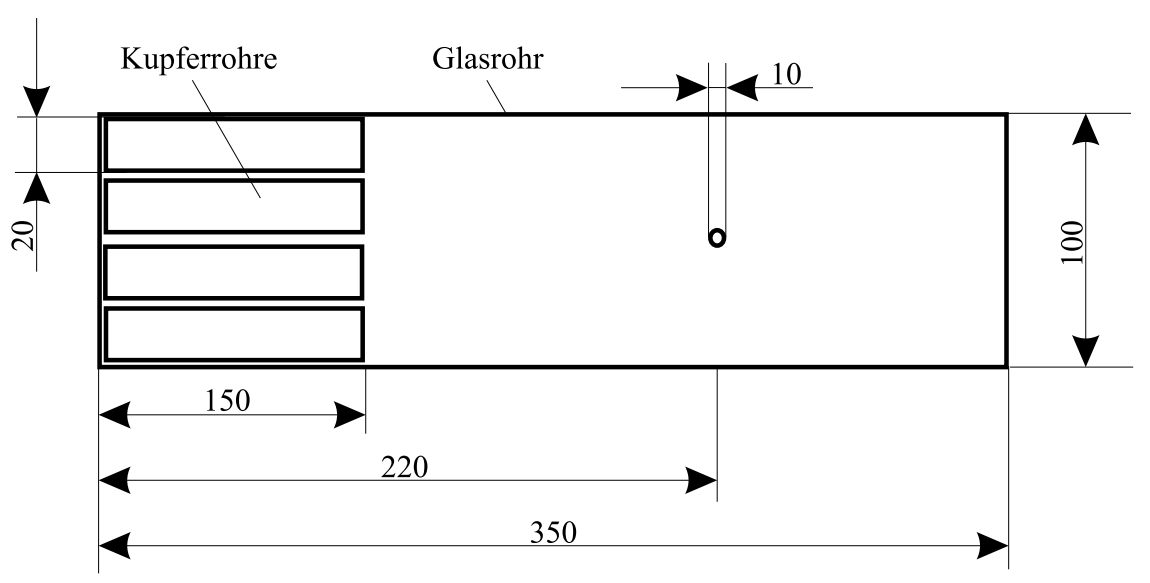

Abbildung 7.6: Apparatur zur Untersuchung des Trocknungsverhaltens von Lösungstropfen am Draht (Maße in $\mathrm{mm}$ )

\section{Versuchdurchführung}

Zunächst wird der Fallturm aufgeheizt, bis der stationäre Zustand erreicht ist. Anschließend wird die Lösung vertropft. Zur Probennahme ist eine Petrischale am Ausgang des Fallturms in den Gasstrom gehalten worden. Die Proben sind anschließend mit einem Lichtmikroskop oder einem Rasterelektronenmikroskop untersucht worden.

\subsubsection{Tropfen am Draht}

In einem Glasrohr mit einem Durchmeser von $100 \mathrm{~mm}$ wird eine feine Kapillare mit einem Außendurchmesser von 0,4 mm aufgehängt, an der wiederum ein Salzlösungstropfen aufgehängt wird. Im Einströmbereich des Gases sind Kupferrohre in das Glasrohr gelegt worden, um eine möglichst gleichmäßige Strömung zu erhalten (Abb. 7.6). Der Heißluftstrom wird mit Hilfe eines Heißluftgebläses erzeugt, das Temperaturen bis $500^{\circ} \mathrm{C}$ erreicht. Eine Regulierung der Temperatur ist durch das Beimischen von kühler Umgebungsluft möglich. Durch die Umgebungsluft kühlt sich der Luftstrom auf die gewünschte Temperatur ab. Mit Hilfe einer Digitalkamera (Anzahl der Pixel: 4 Millionen) werden im Laufe des Trocknungsvorgangs Fotos aufgenommen. Um eine bessere Vergrößerung zu erreichen, wird zusätzlich eine Makrolinse eingesetzt. Für einen detaillierteren Blick in den Trocknungsprozeß ist die Auflösung der Kamera zu gering, weil die Kristalle im trocknenden Tropfen kleiner sind als ein Pixel des CCD-Chips. 

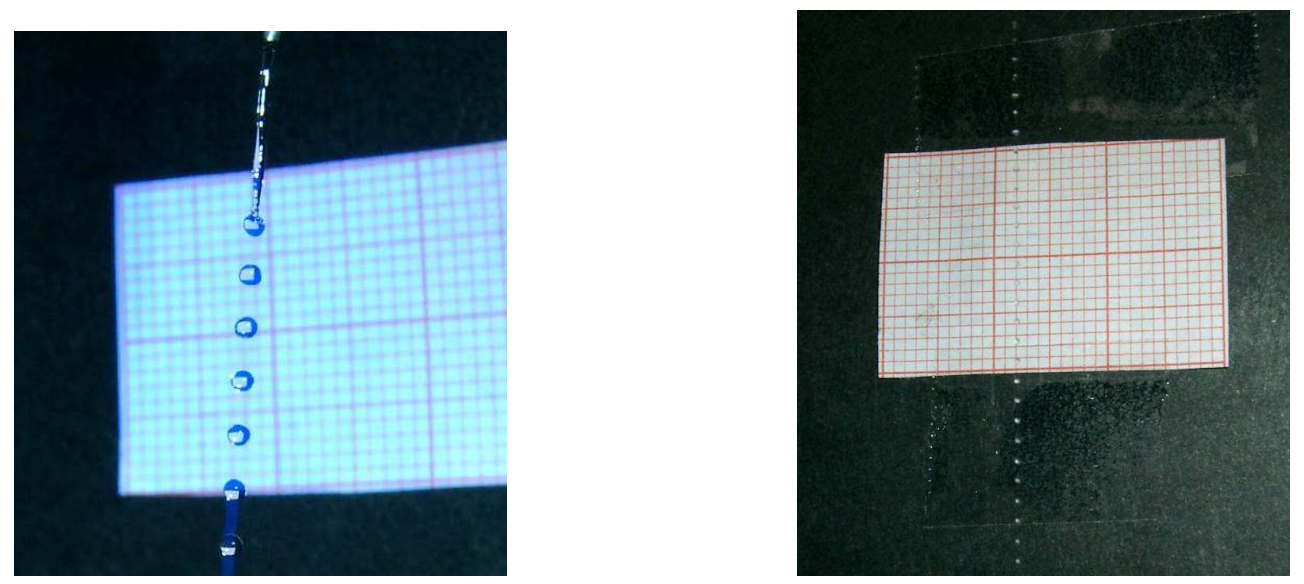

Abbildung 7.7: Wasserstrahlen, die in gleich große Tropfen zerfallen. Links: Kapillardurchmesser $650 \mu \mathrm{m}$; rechts: Kapillardurchmesser $130 \mu \mathrm{m}$. (Zur Skalierung im Hintergrund Millimeterpapier)

\section{$7.2 \quad$ Ergebnisse}

\subsubsection{Versuche am Fallturm}

Im folgenden werden zunächst Ergebnisse zu den Versuchen zum laminaren Strahlzerfall dargestellt. Abb. 7.7 zeigt Wasserstrahlen, die in einzelne gleich große Tropfen zerfallen. Der Durchmesser der Kapillaren beträgt 650 bzw. $130 \mu \mathrm{m}$. Mit Hilfe des Millimeterpapiers läßst sich abschätzen, daß die Tropfen jeweils ungefähr den doppelten Durchmesser der Kapillaren haben. In Abbildung 7.9 sind für einen Strahldurchmesser von $500 \mu \mathrm{m}$ die optimalen Frequenzen für verschiedene Volumenströme aufgetragen. Demnach gibt es für jeden Kapillardurchmesser mehrere optimale Frequenzen. Dies stimmt mit den in [8] dargestellten Beobachtungen überein. Bei einem Strahl mit einem Durchmesser von $130 \mu \mathrm{m}$, wie in Abb. 7.10 dargestellt, sind die Frequenzen entsprechend höher. Bei Verwendung einer Saphirdüse stimmen die theoretisch berechneten und die experimentell ermittelten Frequenzen am besten überein (Abb. 7.11). Abb. 7.12 zeigt, wie die Frequenzen für verschiedene Substanzen variieren. Für Wasser und Salzlösung ist eine deutliche Abweichung der ermittelten Frequenz von der berechneten zu sehen. Dies läßt sich möglicherweise auf Störungen zurückführen, die von der Spitze der Düse hervorgerufen werden. Aufgrund der höheren Viskosität des Silikasols sind die optimalen Frequenzen geringer als bei Wasser oder einer wässrigen Salzlösung.

Es sind verschiedene Lösungen vertropft worden:

- Natriumchlorid

- Ammoniumsulfat 


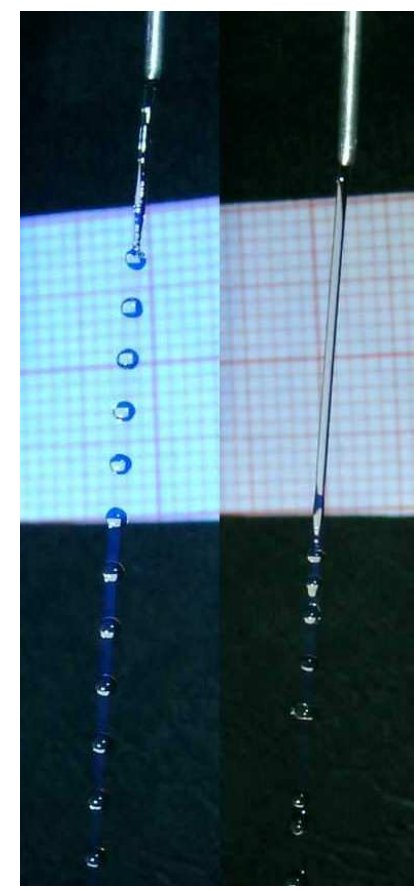

Abbildung 7.8: Strahlzerfall bei Frequenzanregung und natürlicher Strahlzerfall. (Zur Skalierung im Hintergrund Millimeterpapier)

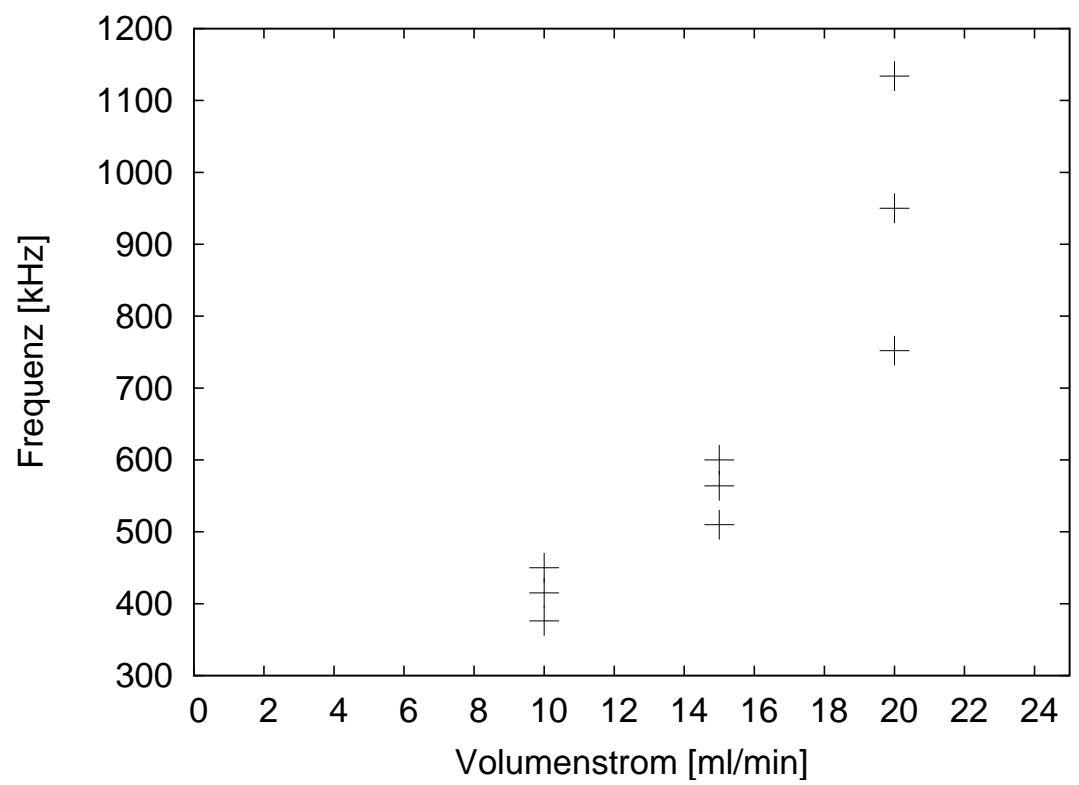

Abbildung 7.9: Frequenzen für gleichmäßigen Strahlzerfall für verschiedene Volumenströme bei einem Strahldurchmesser von $500 \mu \mathrm{m}$ (Flüssigkeit: destilliertes Wasser, Wassertemperatur: $\left.20^{\circ} \mathrm{C}\right)$ 


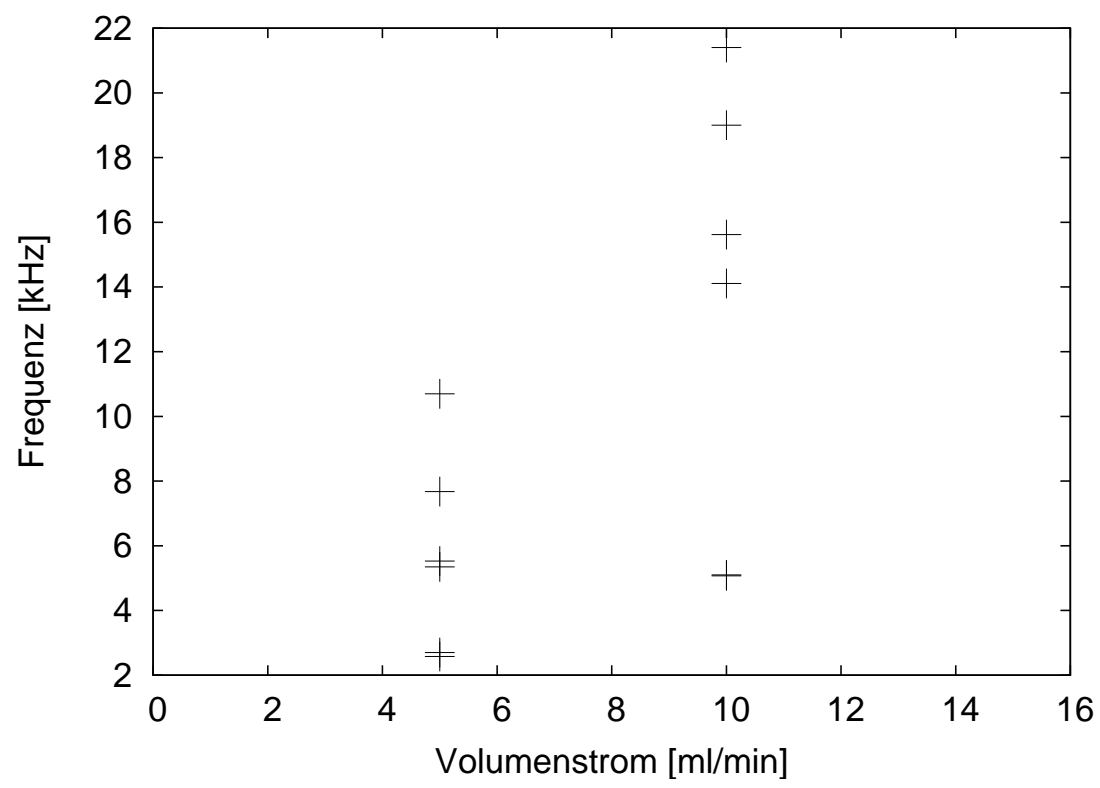

Abbildung 7.10: Frequenzen für gleichmäßigen Strahlzerfall für verschiedene Volumenströme bei einem Strahldurchmesser von $130 \mu \mathrm{m}$ (Flüssigkeit: destilliertes Wasser, Wassertemperatur: $\left.20^{\circ} \mathrm{C}\right)$

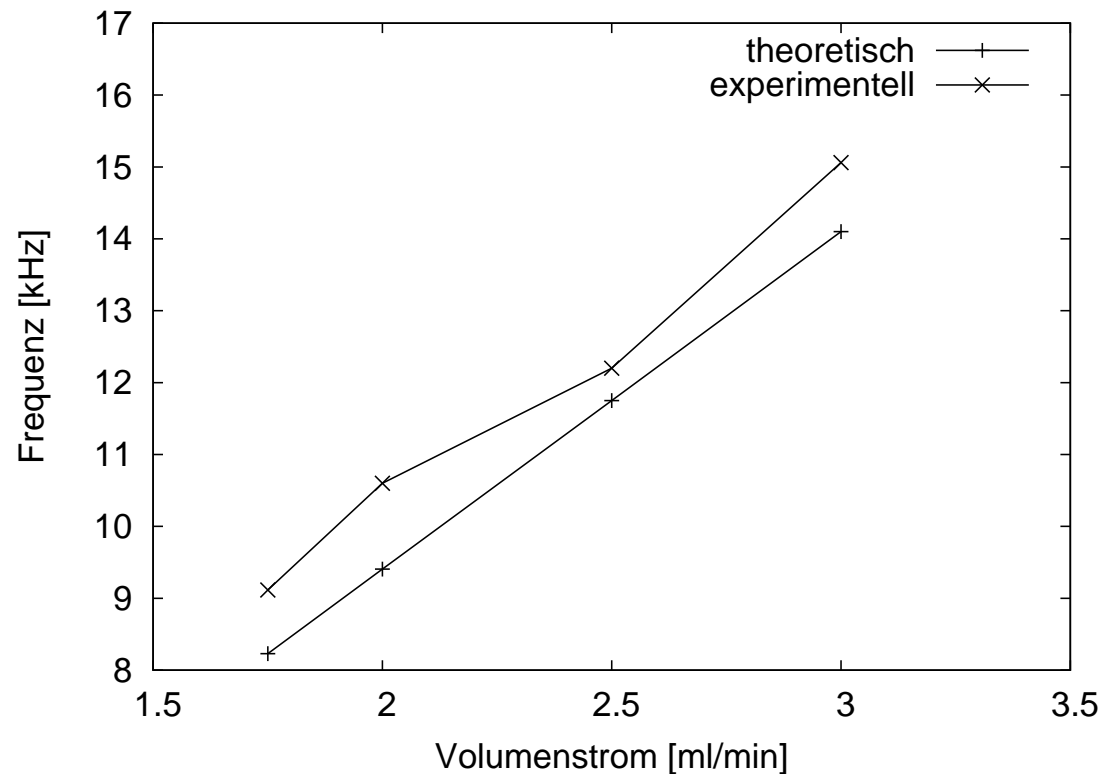

Abbildung 7.11: Vergleich der theoretisch berechneten und experimentell ermittelten optimalen Frequenzen für verschiedene Volumenströme bei Verwendung einer Saphirdüse mit einem Durchmesser von $100 \mu \mathrm{m}$ (Flüssigkeit: destilliertes Wasser, Wassertemperatur: $20^{\circ} \mathrm{C}$ ) 


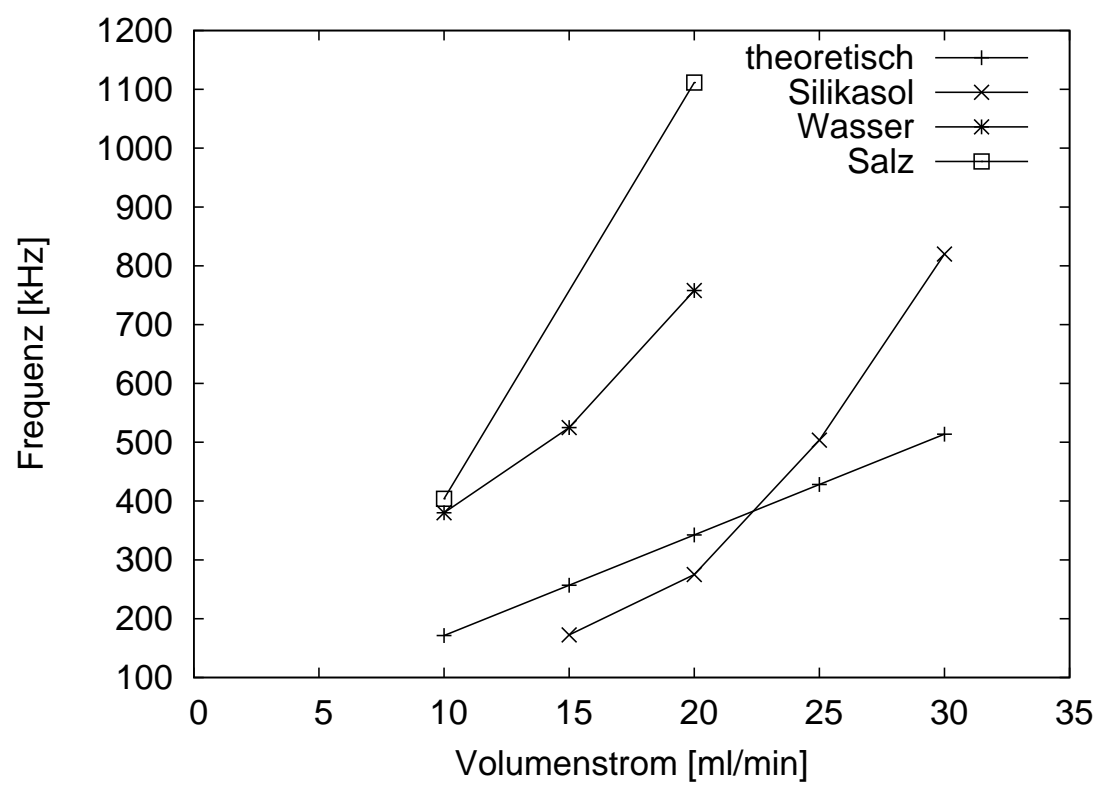

Abbildung 7.12: Frequenzen für gleichmäßigen Strahlzerfall für verschiedene Stoffe und Volumenströme bei einem Strahldurchmesser von $650 \mu \mathrm{m}$ (Flüssigkeitstemperatur: $20^{\circ} \mathrm{C}$; Salz: gesättigte NaCl-Lösung; Silikasol: 40 Gew.-\%, Viskosität $\eta=5,532 \cdot 10^{-2} \mathrm{~Pa}$ s, Oberflächenspannung $50 \cdot 10^{-3} \mathrm{~Pa} \mathrm{~m}$, Dichte $1295 \mathrm{~kg} / \mathrm{m}^{3}$ )

- Kaliumsulfat

- Silikasol

Zum Vertropfen ist eine $200 \mu \mathrm{m}$ Kapillare verwendet worden. Abb. 7.13 zeigt Lichtmikroskopaufnahmen von Salzlösungstropfen, die am Ende des Fallturms aufgefangen worden sind. Deutlich ist die Feststoffbildung um einen noch flüssigen Kern sichtbar.

Bei den Versuchen hat sich jedoch herausgestellt, daß die im Fallturm maximal erreichbare Temperatur von $200^{\circ} \mathrm{C}$ nicht ausreichend ist, um die Tropfen vollständig zu trocknen. Darüber hinaus ist es auf diese Weise nicht möglich, Parameter zu variieren. Für eine weitere Validierung ist ein Fallturm notwendig, in dem Temperaturen bis mindestens $400^{\circ} \mathrm{C}$ erreichbar sind. Eine weitere Verringerung des Ausgangsradius der Tropfen ist nicht möglich, weil die Düsen des Tropfengenerators sehr leicht verstopfen. Ein Düsendurchmesser von $200 \mu \mathrm{m}$ hat sich als kleinster sinnvoller Durchmesser herausgestellt. Bereits bei einem Düsendurchmesser von $100 \mu \mathrm{m}$ sind Experimente kaum noch möglich. Auch ist eine Untersuchung der Struktur der Partikel um so einfacher, je größer sie sind. 

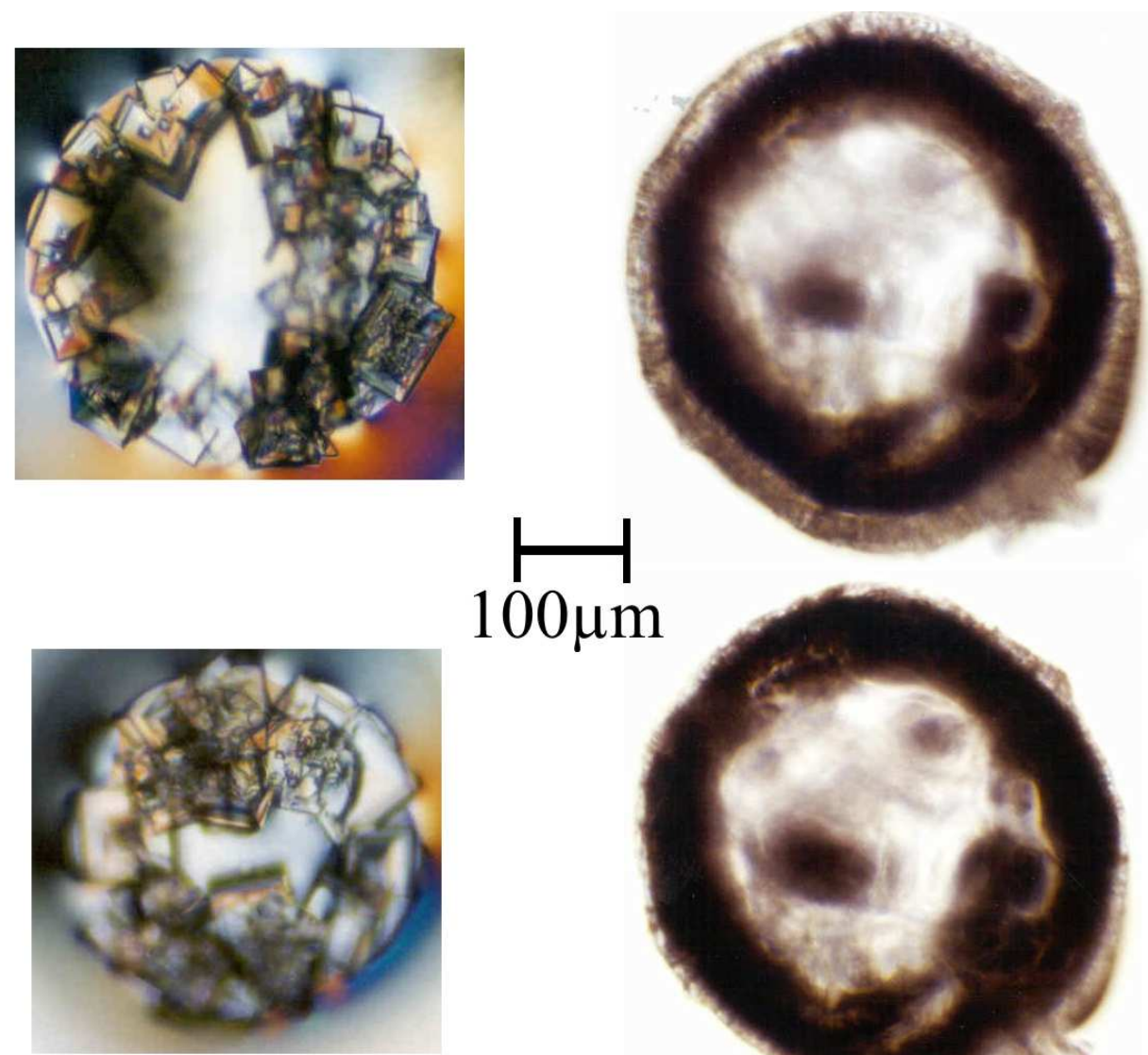
$100 \mu \mathrm{m}$

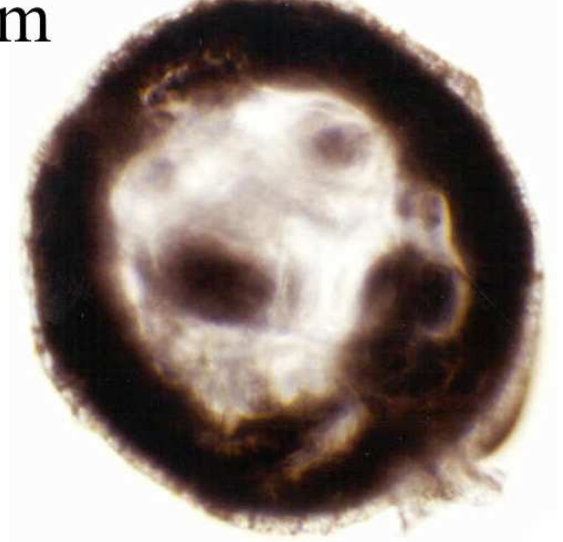

Abbildung 7.13: Mikroskopische Aufnahmen von Partikeln: Links: Bei einer Eingangstemperatur von $200^{\circ} \mathrm{C}$ vertropfte $20 \%$-ige Kochsalzlösung; rechts: Bei einer Eingangstemperatur von $200^{\circ} \mathrm{C}$ vertropfte $43 \%$-ige Ammoniumsulfatlösung 

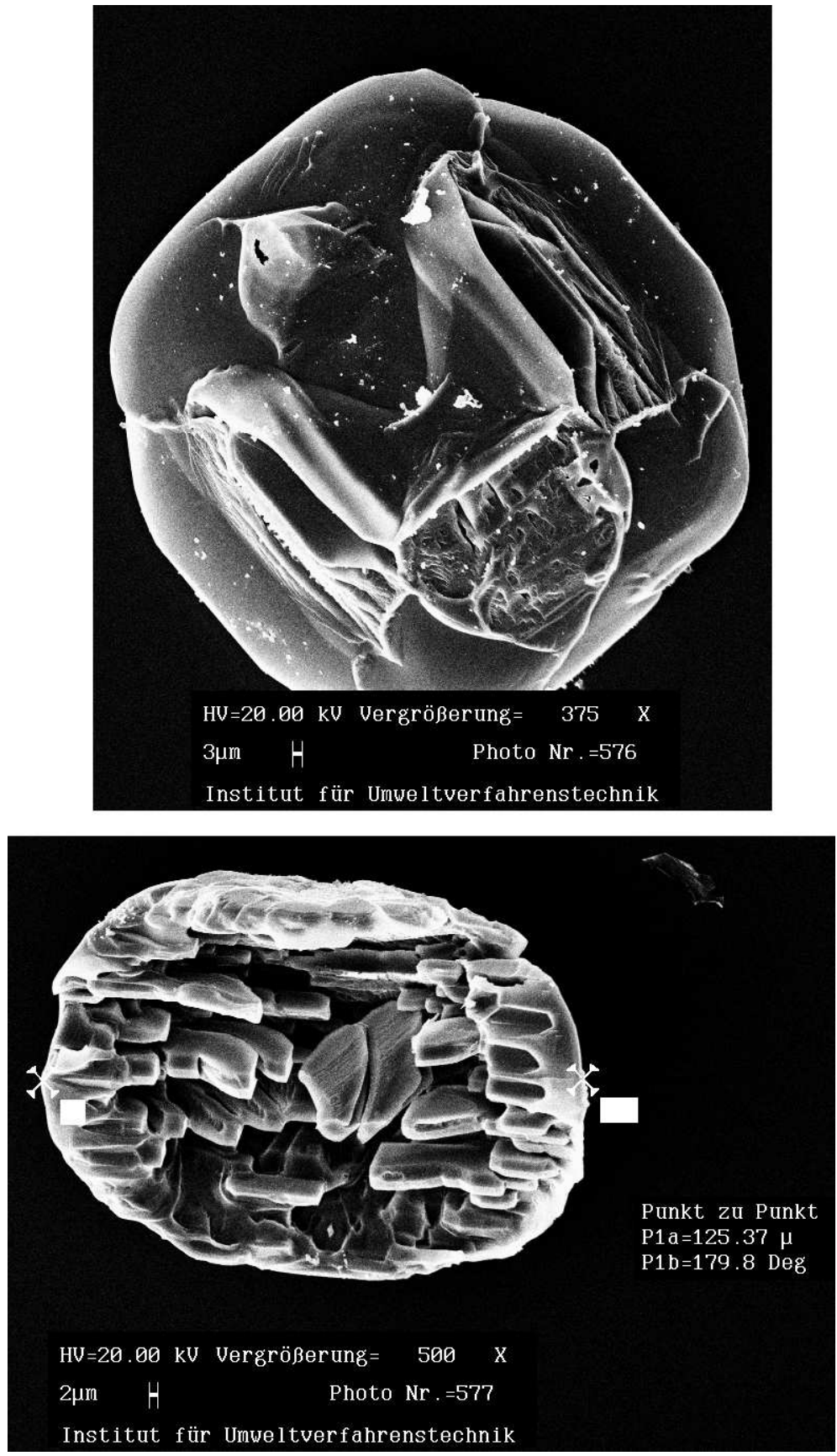

Abbildung 7.14: Elektronenmikroskopische Aufnahmen von bei einer Eingangstemperatur von $200^{\circ} \mathrm{C}$ vertropfter 10\%-iger Ammoniumsulfatlösung (Vergrößerung: Oben: 500 fach, unten 375 fach) 


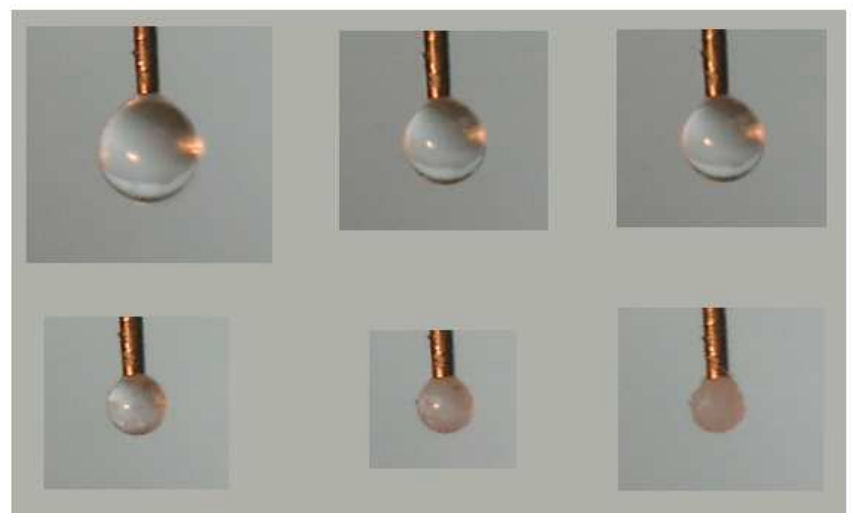

Abbildung 7.15: An einer feinen Kapillare (Außendurchmesser $420 \mu \mathrm{m}$ ) bei einer Temperatur von ca. $200^{\circ} \mathrm{C}$ getrockneter Kaliumsulfattropfen

\subsubsection{Tropfen am Draht}

Abbildung 7.15 zeigt die Trocknung eines Kaliumsulfatlösungstropfens. Der Tropfen schrumpft zunächst aufgrund der Verdunstung des Lösungsmittels. Schließlich beginnt die Bildung von Kristallen, wobei zu erkennen ist, daß sich zunächst an der Tropfenoberfläche Kristalle bilden. Der geringe Radienunterschied bei den letzten beiden Bildern zeigt, daß der Radius nur noch wenig abnimmt, sobald sich eine ausreichend hohe Zahl von Kristallen gebildet hat. Diese Versuche sind zu einem späteren Zeitpunkt der Arbeit durchgeführt worden, nachdem sich die Unzulänglichkeit des Fallturms herausgestellt hat. Wegen der geringen Auflösung der Kamera ist ein detaillierter Blick in die Struktur des getrockneten Tropfens nicht möglich.

\subsection{Zusammenfassung der Versuche}

Es sind Versuche zur Validierung der Simulationsergebnisse durchgeführt worden. Es hat sich in Übereinstimmung mit dem Modell gezeigt, daß bevorzugt Hohlpartikel entstehen. Zur weiteren Validierung des Modells ist es notwendig, Trocknungsversuche mit verschiedenen Anfangsund Randbedingungen durchzuführen. Auf Basis der durchgeführten Versuche scheinen Versuche im Fallturm am günstigsten zu sein, weil die Bedingungen denen, die real im Sprühtrockner herrschen, am nächsten kommen. Die Partikel werden nicht durch einen Draht oder durch Schallwellen wie im Falle des Levitators beeinflußt. Da die Tropfen im Fallturm größer als im Sprühtrockner sind, muß der Einfluß des Anfangstropfenradius untersucht werden. Dies kann durch das Trocknen von Tropfen unterschiedlicher Größe geschehen. Zudem steht für Experimente am Fallturm nun ein ausführlich getesteter, zuverlässiger und kostengünstiger Tropfengenerator zur Verfügung. 


\section{Kapitel 8}

\section{Ergebnisse der Modellrechnungen}

Für eine Vorhersage der Struktur des getrockneten Endprodukts ist es notwendig, den Einfluß der wichtigsten Prozeßparameter auf den Trocknungsprozeß zu untersuchen. Wichtige Parameter bei der Sprühtrocknung sind die Temperatur des Trocknungsgases, die Lösungskonzentration und die Lösungstemperatur, welche Einfluß auf die Sättigungskonzentration hat. Für das Ende des 1. Trocknungsabschnitts, also den Zeitpunkt, wenn der Tropfen komplett von einer festen Hülle umgeben ist, muß als Indikator eine physikalisch sinnvolle Bedingung festgelegt werden. Bei einem Partikelvolumenanteil am Tropfenrand von $74 \%$ sind die Partikel am dichtesten gepackt, so daß eine weitere Bewegung nach innen und somit eine weitere Komprimierung nicht möglich ist. Daher wird das Ende des 1. Trocknungsabschnitts als Zeitpunkt definiert, an dem am Tropfenrand der Partikelvolumenanteil $74 \%$ beträgt. Da Luft ein weit verbreitetes Trocknungsgas ist, wird für die Berechnungen Luft als Trocknungsgas verwendet, wobei angenommen wird, daß die Luft keinen Wasserdampf enthält. Die Partikelanzahldichte beschreibt die Partikelkonzentration in einem vierdimensionalen Raum: Die Partikelanzahldichte wird je physikalischem Tropfenvolumen und je Partikellänge/-größe be-

rechnet. Da das Modell radialsymmetrisch ist, entfallen zwei der drei räumlichen Dimensionen, und der Radius bleibt als einzige räumliche Dimesion übrig. Daher hat die Partikelanzahldichte die Einheit $1 / \mathrm{m}^{4}$. $\mathrm{n}$ ist zum einen eine Verteilung und zum anderen eine Konzentration, weil $n$ die Partikelzahl pro $\mathrm{m}^{3}$ darstellt. Durch Integration von $n$ über der Partikellänge L und dem Schalenvolumen im Tropfen ergibt sich die Partikelzahl in einer Schale.

Abb. 8.1 zeigt die zeitliche Änderung des quadrierten Tropfendurchmessers. Der Tropfen schrumpft zunächst gemäß dem in Kapitel 3.1.5 erläuterten $d^{2}$-Gesetz, der quadrierte Durchmesser nimmt also linear mit der Zeit ab. Sobald sich eine feste Hülle gebildet hat, bleibt der Durchmesser konstant. Lediglich zu Beginn des Trocknungsprozesses ist die Radienabnahme nicht linear. Dies ist auf die Erwärmung des Tropfens auf Kühlgrenztemperatur zurückzuführen (Abb. 8.2). Die schraffierten Bereiche zwischen den Kurven in Abb. 8.2 zeigen die 


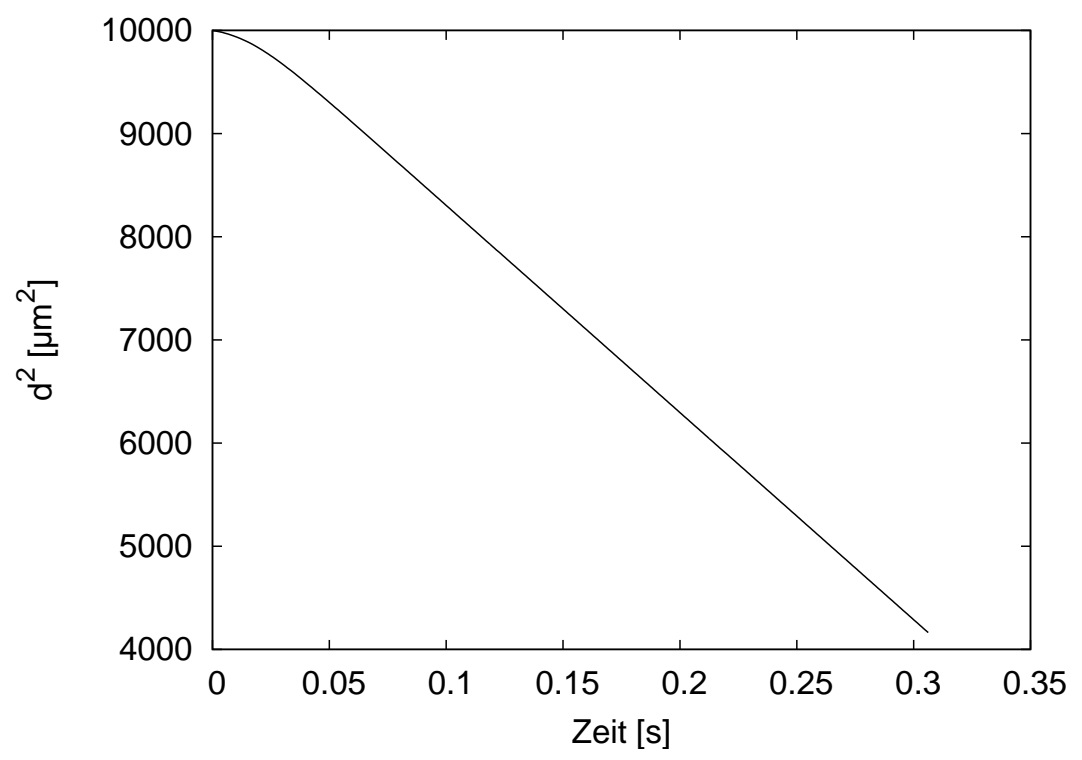

Abbildung 8.1: Zeitliche Abnahme des quadrierten Tropfendurchmessers.

(Massenanteil der gelösten Substanz $w_{G}(t=0 s)=0,09$, Dichte der gelösten Substanz $\rho_{G}^{\prime}=2670 \mathrm{~kg} / \mathrm{m}^{3}$, Dichte des Lösungsmittels $\rho_{L}^{\prime}=1000 \mathrm{~kg} / \mathrm{m}^{3}$, Tropfentemperatur $T_{\text {Tropfen }}(t=0 s)=293 \mathrm{~K}$, Wärmekapazität $c_{p_{\text {Wasser }}}=4,183 \mathrm{~kJ} /(\mathrm{kgK})$, Wärmekapazität $c_{p_{G}}=0,92 \mathrm{~kJ} /(\mathrm{kgK})$, Wärmeleitfähigkeit $\lambda_{\text {Tropfen }}=0,637 \mathrm{~W} /\left(\mathrm{m}^{2} \mathrm{~K}\right)$, Wärmeleitfähigkeit $\lambda_{\text {Luft }}=0,03795 \mathrm{~W} /\left(\mathrm{m}^{2} \mathrm{~K}\right)$, Umgebungstemperatur $\left.T_{U}=473 \mathrm{~K}\right)$

Enthalpie, die der Tropfen durch Verdunstung abgegeben hat und die dem Tropfen aus dem Trocknungsgas zugeführt worden ist.

Die Abb. 8.3 und 8.4 zeigen exemplarisch den Temperaturverlauf in einem $\mathrm{K}_{2} \mathrm{SO}_{4}$-Lösungstropfen, der bei einer Lufttemperatur von 473K getrocknet wird, wobei die Anfangstemperatur der Lösung 293K beträgt. Die Temperatur im Tropfen steigt während des 1. Trocknungsabschnitts zunächst an und bleibt anschließend nach Erreichen der Kühlgrenztemperatur konstant. Der radiale Temperaturverlauf im Tropfen ist wegen der geringen Größe des Tropfens und des geringen Wärmetransportwiderstands im Tropfen im Vergleich zum Wärmetransportwiderstand in der Gasphase sehr gleichmäßig. Dies läßt sich z.B. mit der Biot-Zahl abschätzen

$$
B i=\frac{\alpha \cdot d}{\lambda_{\text {Tropfen }}},
$$

die für Tropfen mit einem Radius von $100 \mu \mathrm{m}$ kleiner als 0,1 ist. Die maximale Temperaturdifferenz kann mit

$$
\Delta \Theta_{\max }=\frac{B i}{1+B i}
$$

abgeschätzt werden [72]. Bei einer Wärmeleitfähigkeit des Wassers von $\lambda=0,667 \mathrm{~W} /(m K)$ und einem Wärmeübergangskoeffizienten von $\alpha=379 \mathrm{~W} /\left(\mathrm{m}^{2} \mathrm{~K}\right)$ beträgt die maximale Tem- 


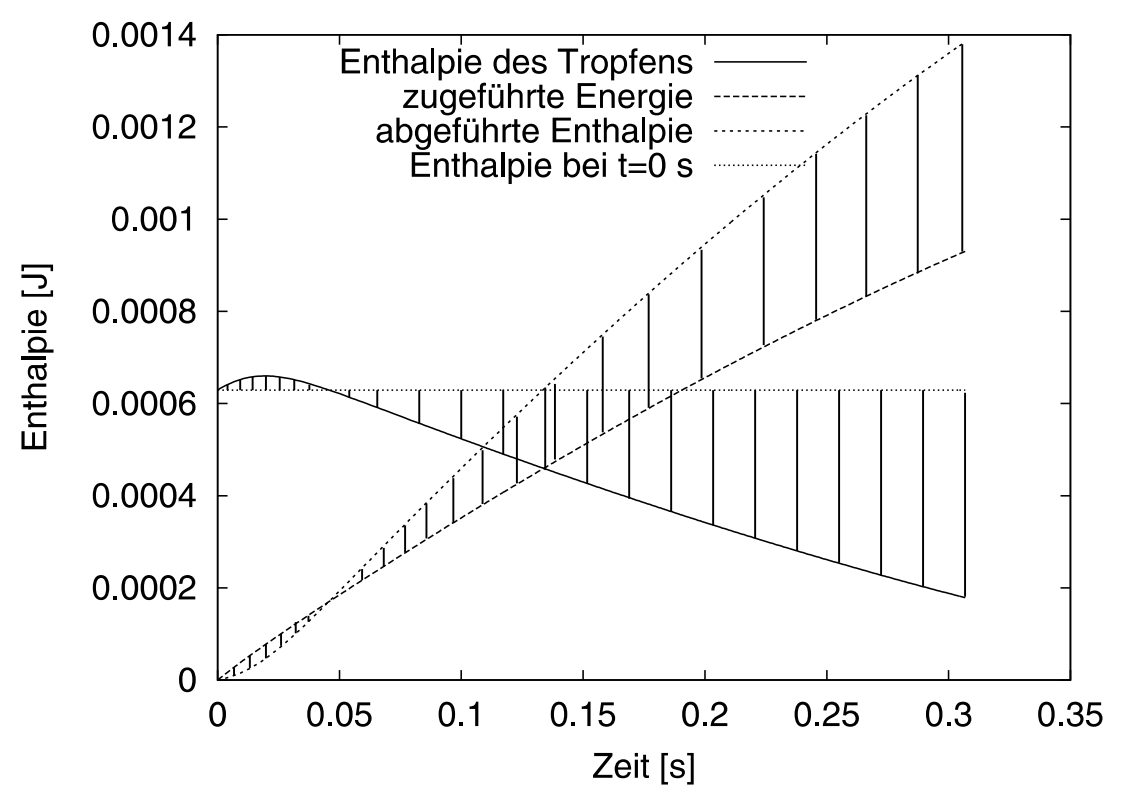

Abbildung 8.2: Energiebilanz um einen $\mathrm{K}_{2} \mathrm{SO}_{4}$-Lösungstropfen. (Parameter siehe Abb. 8.1)

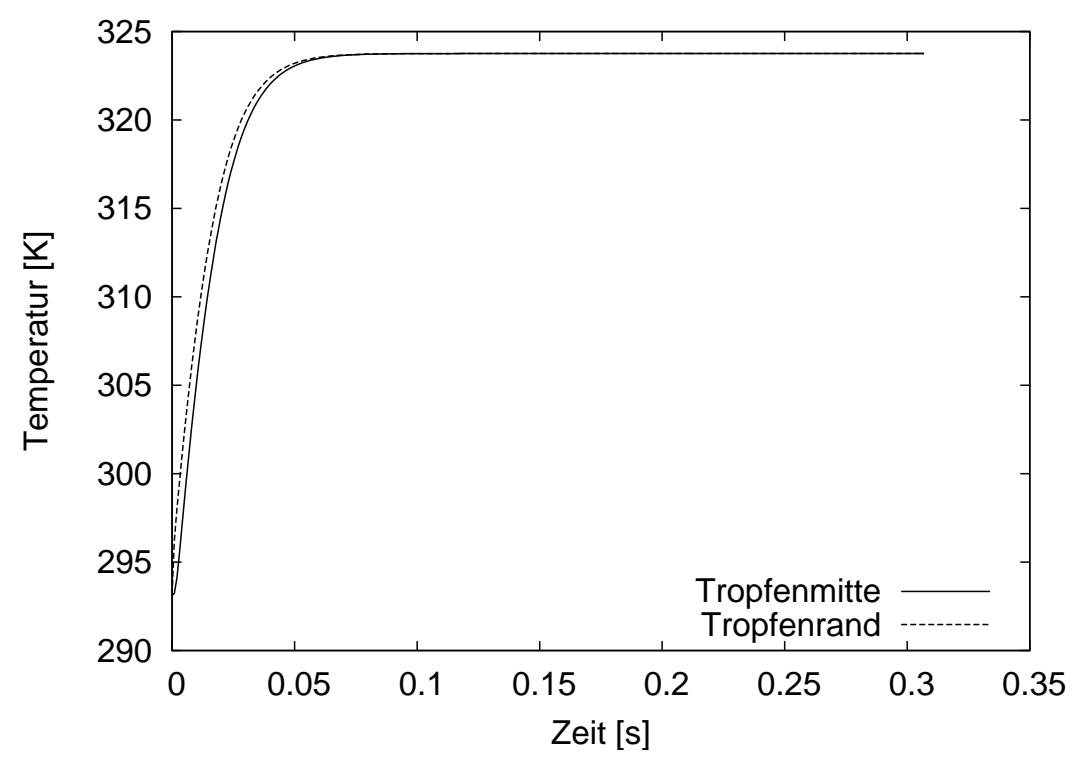

Abbildung 8.3: Zeitlicher Verlauf der Temperatur im Tropfenmittelpunkt und am Tropfenrand (Parameter siehe Abb. 8.1)

peraturdifferenz zwischen Tropfenrand und Tropfenmittelpunkt 10\%, was mit den Simulationsergebnissen übereinstimmt. Darüber hinaus ist zu erkennen, daß der Tropfen die Kühlgrenztemperatur innerhalb von Bruchteilen einer Sekunde erreicht hat, was ebenfalls auf den geringen Wärmetransportwiderstand im Tropfen zurückzuführen ist. 


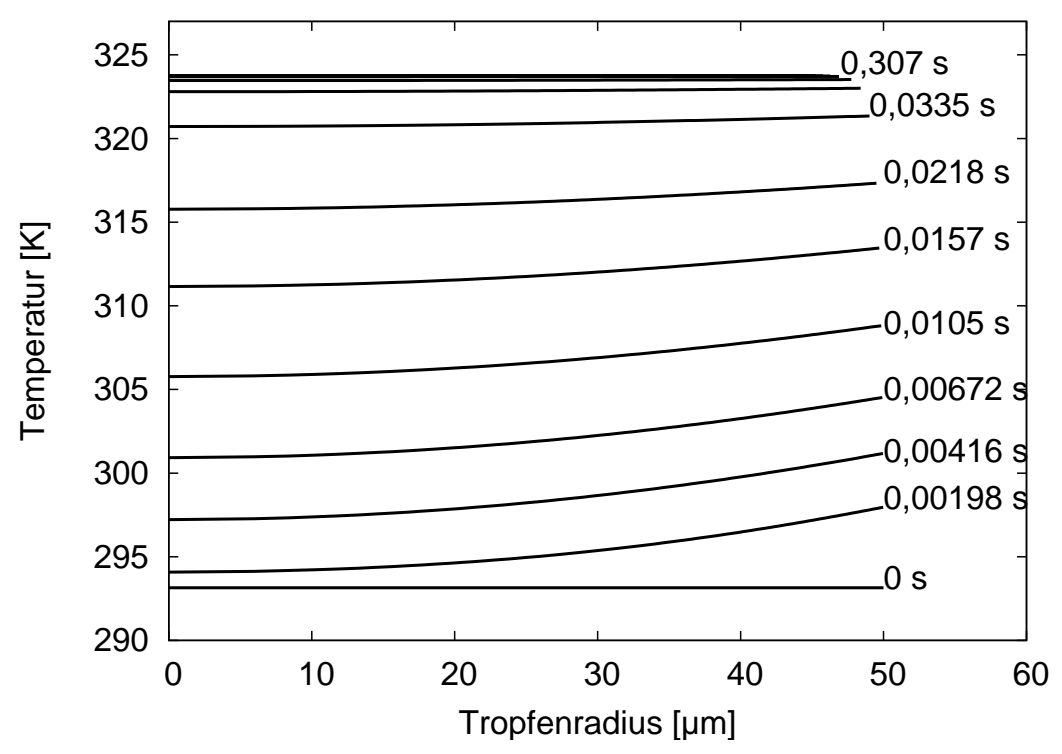

Abbildung 8.4: Zeitlicher und radialer Verlauf der Temperatur im Tropfen (Parameter siehe Abb. 8.1)

\subsection{Trocknung von Suspensionstropfen}

Zunächst soll das Verhalten von Suspensionstropfen modelliert werden. Die Partikelgröße in kolloidalen Suspensionen liegt im Nanometerbereich. Die Partikel im Silikasol haben eine Größe von ungefähr 7 bis $8 \mathrm{~nm}$. Abb. 8.5 zeigt die Anzahldichteverteilung am Ende des ersten Trocknungsabschnitts bei einer Trocknungslufttemperatur von 473K und einer Anfangstemperatur der Suspension von 293K. Deutlich ist die sich ausbildende Hülle zu erkennen. Im Falle einer 40 Vol.-\%-igen Suspension bildet sich eine deutlich dickere Hülle. Für beide Rechnungen ist angenommen worden, daß ab einem Volumenanteil von 55 Vol.-\% in der Schale, die die Partikel aufsammelt, keine Partikel mehr hineinpassen und die Partikelanzahldichte nur noch durch die Volumenabnahme zunimmt.

Wie bereits in Kapitel 2.6 beschrieben, haben Liang et al. ein Modell [26] für den Trocknungsverlauf eines Suspensionstropfens während des 1. Trocknungsabschnitts entwickelt. Dem Modell zufolge ist das Partikel am Ende des 1. Trocknungsabschnitts umso größer, je höher der Anfangsvolumenanteil der Partikel in der Suspension ist. Dies läßt sich mit diesem Modell bestätigen (Abb. 8.7). Nach Walton und Mumford [65] entstehen aus sprühgetrockneten Suspensionstropfen mit großen Suspensionspartikeln Vollpartikel, während aus solchen mit kleinen Suspensionpartikeln Hohlpartikel entstehen. Daher erscheint es gerechtfertigt, Partikeldiffusion nicht zu berücksichtigen, weil Partikeldiffusion vor allen Dingen bei sehr kleinen Partikeln im Nanometerbereich auftritt. Auch ist anzunehmen, daß bei sehr kleinen Suspensionspartikeln sich im Laufe des Trocknungsprozesses eine Gasblase im Inneren ausbildet, weil 


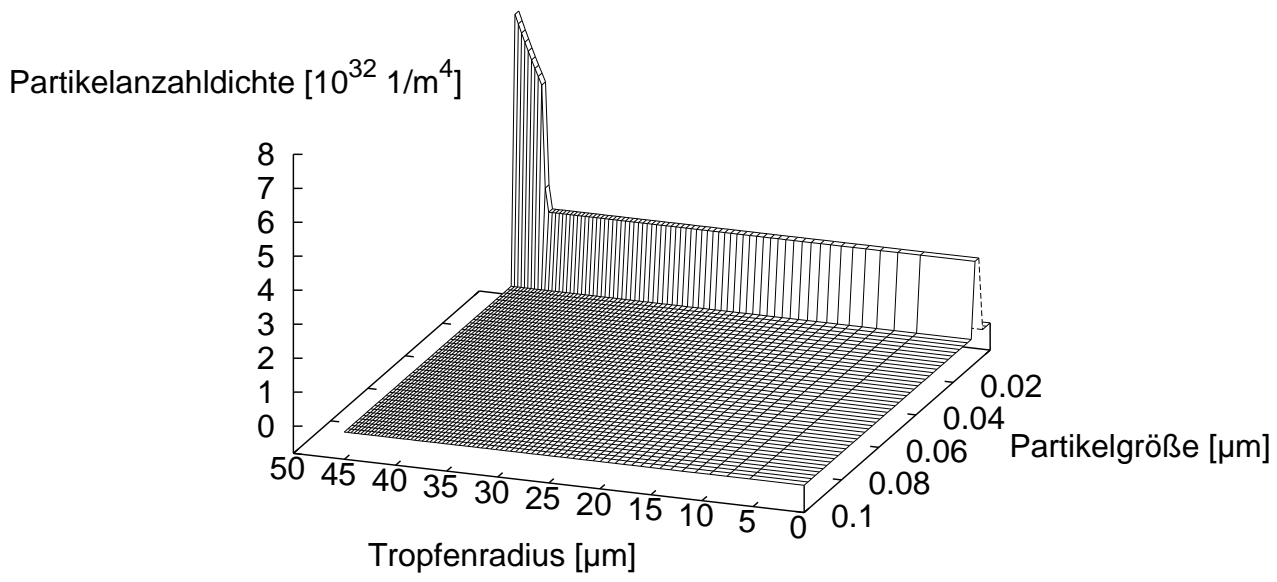

Abbildung 8.5: Partikelanzahldichteverteilung in einem wäßrigen Suspensionstropfen (20 Vol.\%) über dem Tropfenradius und der Partikelgröße am Ende des 1. Trocknungsabschnitts (473K Lufttemperatur)

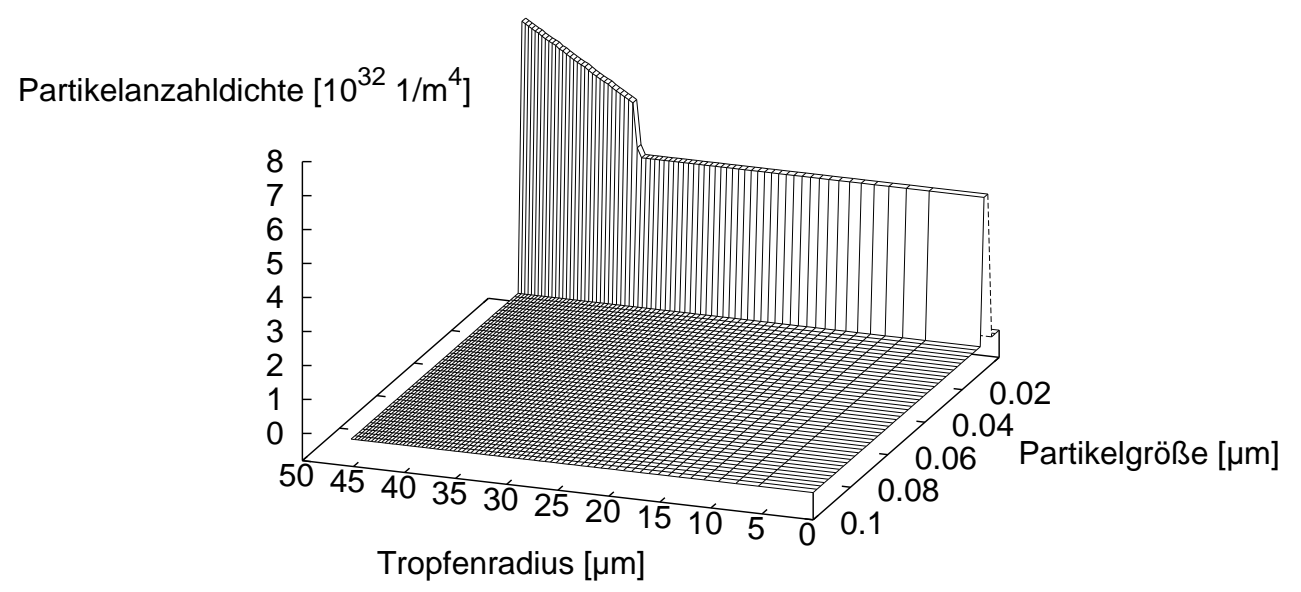

Abbildung 8.6: Partikelanzahldichteverteilung in einem wäßrigen Suspensionstropfen (40 Vol.$\%$ ) über dem Tropfenradius und der Partikelgröße am Ende des 1. Trocknungsabschnitts (473K Lufttemperatur)

die Zwischenräume zwischen den Partikeln sehr viel kleiner sind und somit der Abtransport des Dampfes behindert wird. Mit der Bildung der Gasblase ist dann die Hohlraumbildung im Inneren verbunden. 


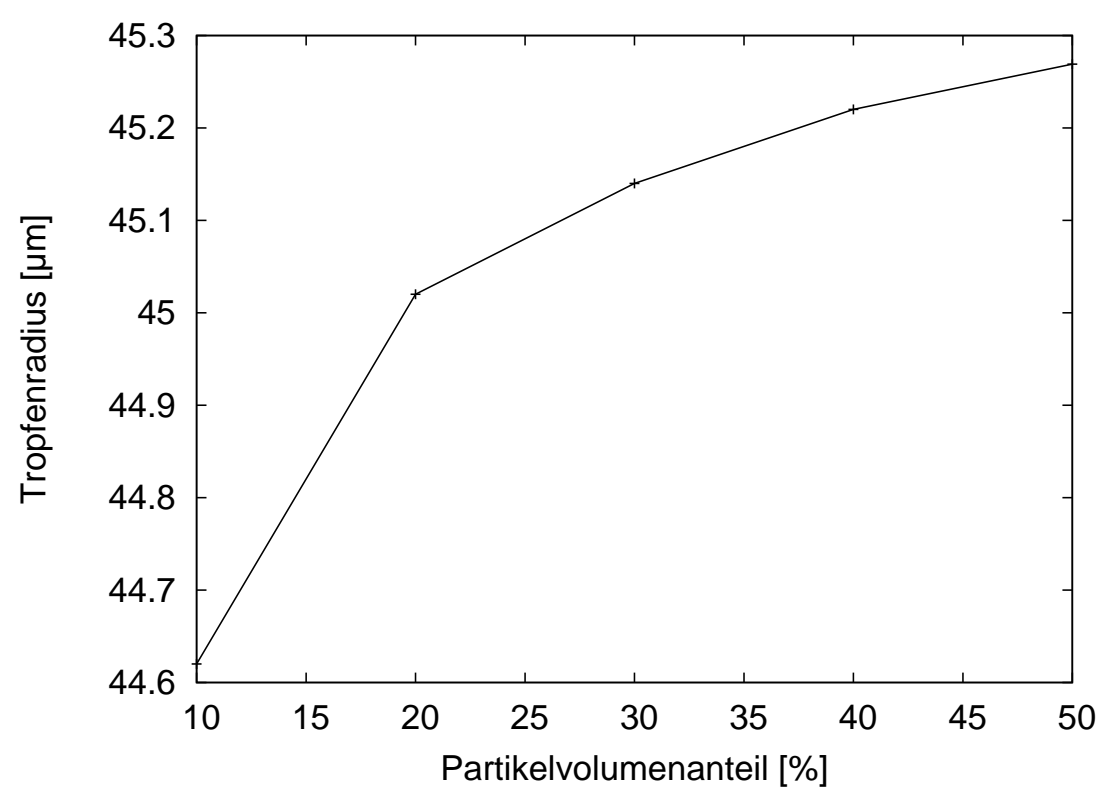

Abbildung 8.7: Radius eines Suspensionstropfens mit einem Anfangsradius von $50 \mu \mathrm{m}$ am Ende des 1 . Trocknngsabschnitts für verschiedene Partikelvolumenanteile

\subsection{Trocknung von Lösungstropfen}

Zur Untersuchung der Abhängigkeit der Morphologie des Endproduktes in Abhängigkeit von Prozeßparametern werden im folgenden Simulationsrechnungen mit Kaliumsulfat- und Ammoniumsulfatsalzlösungen dargestellt.

Die Keimbildung $B$ und das Wachstum $G$ von Kaliumsulfat kann nach [73] mit

$$
\begin{gathered}
B=6,67 \cdot 10^{17}\left(\frac{w_{G}}{w_{L}}\right)^{7,63} \cdot \rho_{\text {Sus }} \\
G=0,0467 \cdot L^{0,5}\left(\frac{w_{G}}{w_{L}}\right)^{2,0}
\end{gathered}
$$

berechnet werden, wobei die Wachstumsgeschwindigkeit von der Partikelgröße L abhängig ist. Es werden in der Literatur keine Angaben darüber gemacht, ob die Kinetik für die homogene oder heterogene Kristallisation gilt. Die Sättigungskonzentration beträgt bei $293 \mathrm{~K}$ $111 \mathrm{~kg} / \mathrm{m}^{3}$, bei $323 \mathrm{~K} 165 \mathrm{~kg} / \mathrm{m}^{3}$ und bei $373 \mathrm{~K} 241 \mathrm{~kg} / \mathrm{m}^{3}$ [74]. Aus diesen Werten wird eine Ausgleichsgerade berechnet

$$
\rho_{\text {Sat }}=1,6132 \cdot T-359,44
$$

mit der die Temperaturabhängigkeit der Löslichkeit berücksichtigt wird.

Da es im Falle von Ammoniumsulfat keine empirischen Kinetiken in der Literatur gibt, werden hier Keimbildung und Wachstum mit Kinetiken nach [75] berechnet. Für den Fall 
homogener Keimbildung lautet die Kinetik

$$
\begin{gathered}
B=F \cdot E \\
\operatorname{mit} F=\frac{3}{2} D_{A B} d_{m}^{2}\left(c N_{A}\right)^{\frac{7}{3}} \sqrt{k \ln \left(\frac{c_{c}}{c^{*}}\right)} \\
\text { und } E=\exp \left(-\frac{16}{3}\left(k \ln \left(\frac{c_{c}}{c^{*}}\right)\right)^{3} \frac{1}{\left(\nu \ln S_{a}\right)^{2}}\right) .
\end{gathered}
$$

Darin ist $D_{A B}$ der Diffusionskoeffizient, $N_{A}$ die Avogadro-Zahl, k die Boltzmann-Konstante, $c_{c}$ die Cluster-Konzentration, $c^{*}$ die Löslichkeit und $\nu$ ein stöchiometrischer Koeffizient. Das Wachstum der Kristalle berechnet sich aus

$$
\begin{gathered}
G=2 \cdot\left(v_{B C F}+v_{B+S}+v_{v_{P N}}\right) \\
\text { mit } \\
v_{B C F}=2,25 \cdot 10^{-3} \nu^{2} \frac{D_{A B}}{d_{m}} \frac{\left(\frac{c^{*}}{c_{c}}\right)^{4 / 3}}{\ln \left(\frac{c_{c}}{c^{*}}\right)} \sigma^{2} \\
v_{B+S}=\left(\frac{2}{\pi}\right)^{1 / 3} \frac{D_{s}}{d_{m}}\left(\frac{c^{*}}{c_{c}}\right)^{3 / 2}[\ln (1+\sigma)]^{1 / 6} \cdot \exp \left(-\frac{\frac{\pi}{3}\left[K \ln \left(\frac{c_{c}}{c^{*}}\right)\right]^{2}}{\nu \ln (1+\sigma)}\right) \\
v_{P N}=\frac{D_{A B}}{3 d_{m}}\left(\frac{c^{*}}{c_{c}}\right)^{2 / 3} \exp \left(-\frac{\pi\left[k \ln \left(\frac{c_{c}}{c^{*}}\right)\right]^{2}}{\nu \ln (1+\sigma)}\right),
\end{gathered}
$$

wobei $D_{S}$ der Oberflächendiffusionskoeffizient ist und $\sigma$ die relative Übersättigung. Unter der Annahme, daß das Wachstum nicht durch die Wärmeübertragung und die Massendiffusion beeinflußt wird, setzt sich die Wachstumsgeschwindigkeit gemäß Gl. (8.6) additiv aus den Geschwindigkeiten der verschiedenen Wachstumsmechanismen zusammen [75]. Die Stoffdaten zur Berechnung der Kristallisation sind aus [41] entnommen. Der stöchiometrische Koeffizient $\nu$ ist 2, der Volumen- und Oberflächendiffusionskoeffizient $9,16 \cdot 10^{-10} \mathrm{~m}^{2} / \mathrm{s}$, der Moleküldurchmesser $1 \cdot 10^{-10} \mathrm{~m}$ und das Verhältnis von Clusterkonzentration zu Löslichkeit 0,3. Die Sättigungsgrenze des Ammoniumsulfats liegt bei $293 \mathrm{~K}$ bei $535,13 \mathrm{~kg} / \mathrm{m}^{3}$ [41]. Da die Löslichkeit nur schwach temperaturabhängig ist, werden die Berechnungen bei konstanter Sättigungsgrenze durchgeführt.

Am Beispiel der Kaliumsulfatkinetik ist in den Abb. 8.8 und 8.9 (als Fortsetzung von Abb. 8.8) die zeitliche Änderung der Partikelanzahldichte über dem Tropfenradius und der Partikellänge dargestellt. Deutlich zu erkennen ist, daß sich die Partikel am Tropfenrand konzentrieren. Dies ist darauf zurückzuführen, daß die Kristallisation am Tropfenrand beginnt, weil der Massenanteil der gelösten Substanz wegen der Verdunstung des Lösungsmittels zunächst am Tropfenrand die Sättigungsgrenze überschreitet. Darüber hinaus sammelt die Zelle am Tropfenrand die Partikel auf, die sich weiter innen befinden, sobald sich die Phasengrenze über sie hinweg bewegt. Das Ende des 1. Trocknungsabschnitts ist nach 0,134 s erreicht. Abb. 
8.10 zeigt den zeitlichen Verlauf der radialen Verteilung des Massenanteils der gelösten Substanz. Während der Schrumpfung des Tropfens steigt der Massenanteil zunächst an. Durch die Kristallisation fällt der Massenanteil dann jedoch wieder ab, weil das Salz aus der flüssigen Phase in die feste Phase übergeht. Die Massenanteile der gelösten Substanz und des Lösungsmittels beziehen sich auf die Gesamtmasse der Flüssigkeit. Die feste Phase ist bereits durch den Volumenanteil $\epsilon$ in der Massenbilanz berücksichtigt. 

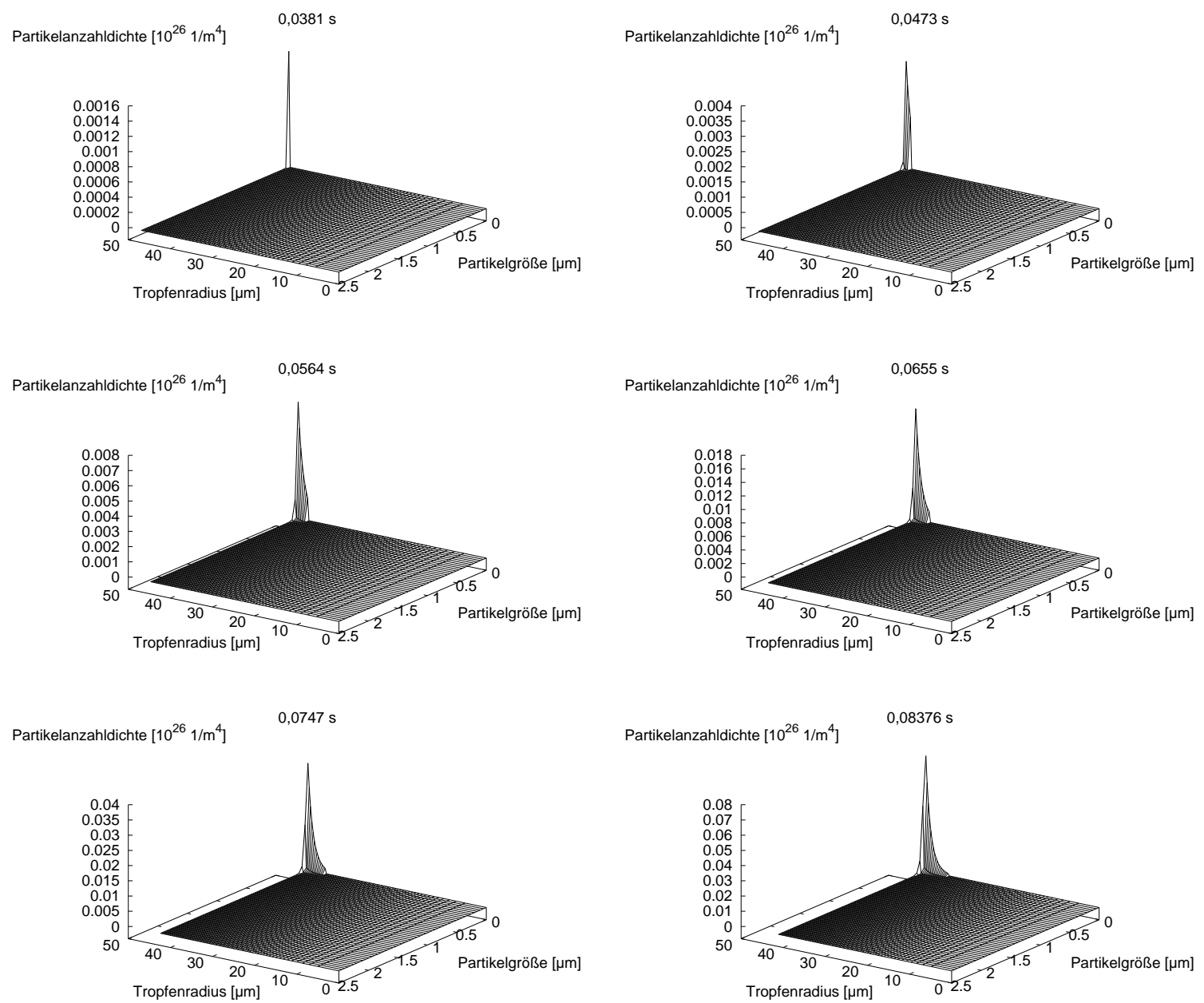

Abbildung 8.8: Partikelanzahldichte in einem $\mathrm{K}_{2} \mathrm{SO}_{4}$-Lösungstropfen über dem Tropfenradius und der Partikelgröße zu verschiedenen Zeitpunkten der Trocknung

(Massenanteil der gelösten Substanz $w_{G}(t=0 s)=0,09$, Dichte der gelösten Substanz $\rho_{G}^{\prime}=$ $2670 \mathrm{~kg} / \mathrm{m}^{3}$, Dichte des Lösungsmittels $\rho_{L}^{\prime}=1000 \mathrm{~kg} / \mathrm{m}^{3}$, Tropfentemperatur $T_{\text {Tropfen }}(t=$ $0 s)=293 K$, Wärmekapazität $c_{p_{\text {Wasser }}}=4,183 \mathrm{~kJ} /(\mathrm{kgK})$, Wärmekapazität $c_{p_{G}}=$ $0,92 \mathrm{~kJ} /(\mathrm{kgK})$, Wärmeleitfähigkeit $\lambda_{\text {Tropfen }}=0,637 \mathrm{~W} /\left(\mathrm{m}^{2} \mathrm{~K}\right)$, Wärmeleitfähigkeit $\lambda_{\text {Luft }}=$ $0,03795 \mathrm{~W} /\left(\mathrm{m}^{2} \mathrm{~K}\right)$, Luftemperatur $\left.T_{U}=573 \mathrm{~K}\right)$ 

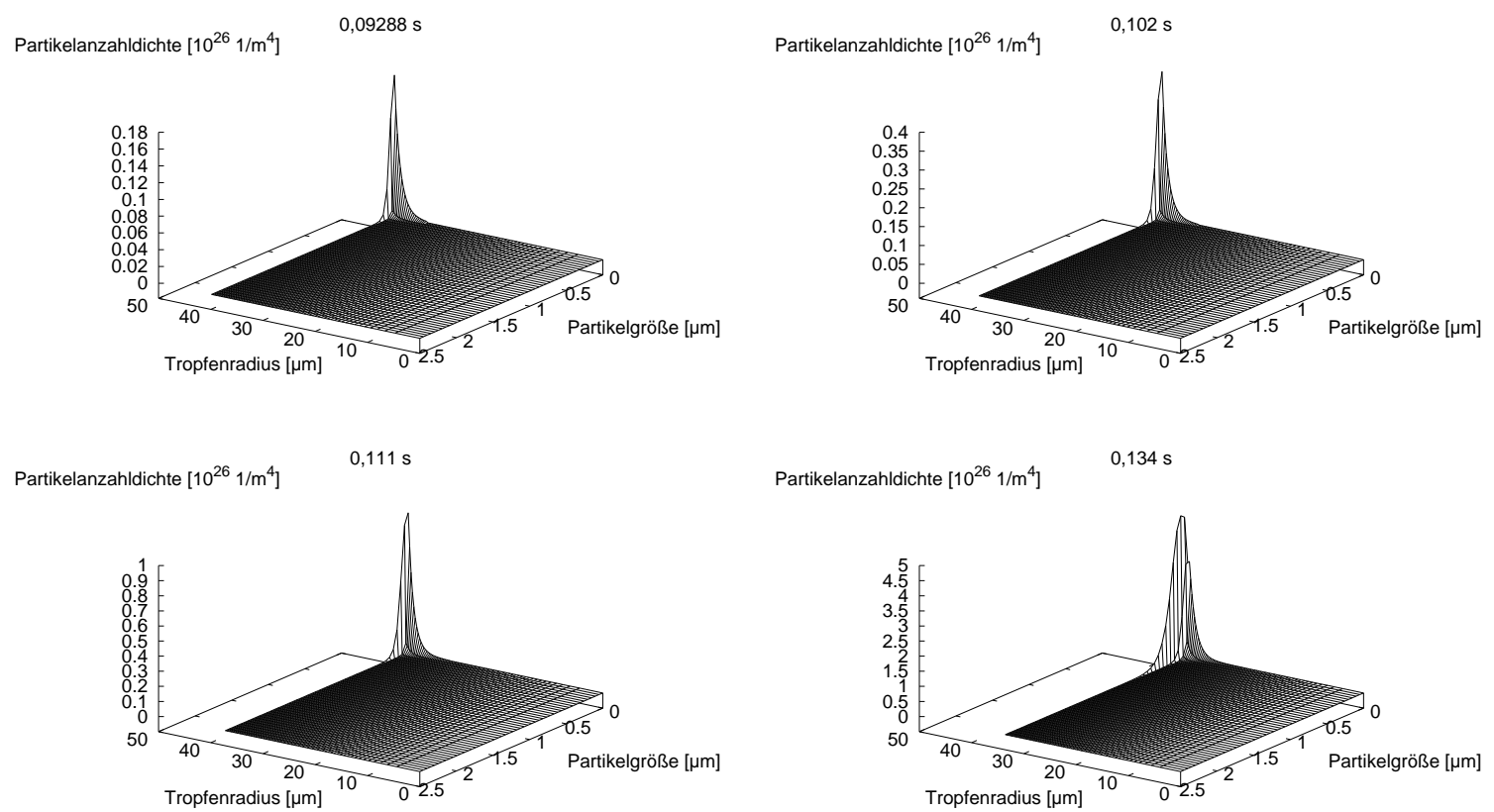

Abbildung 8.9: Partikelanzahldichte in einem $\mathrm{K}_{2} \mathrm{SO}_{4}$-Lösungstropfen über dem Tropfenradius und der Partikelgröße zu verschiedenen Zeitpunkten der Trocknung (Parameter siehe Abb. $8.8)$

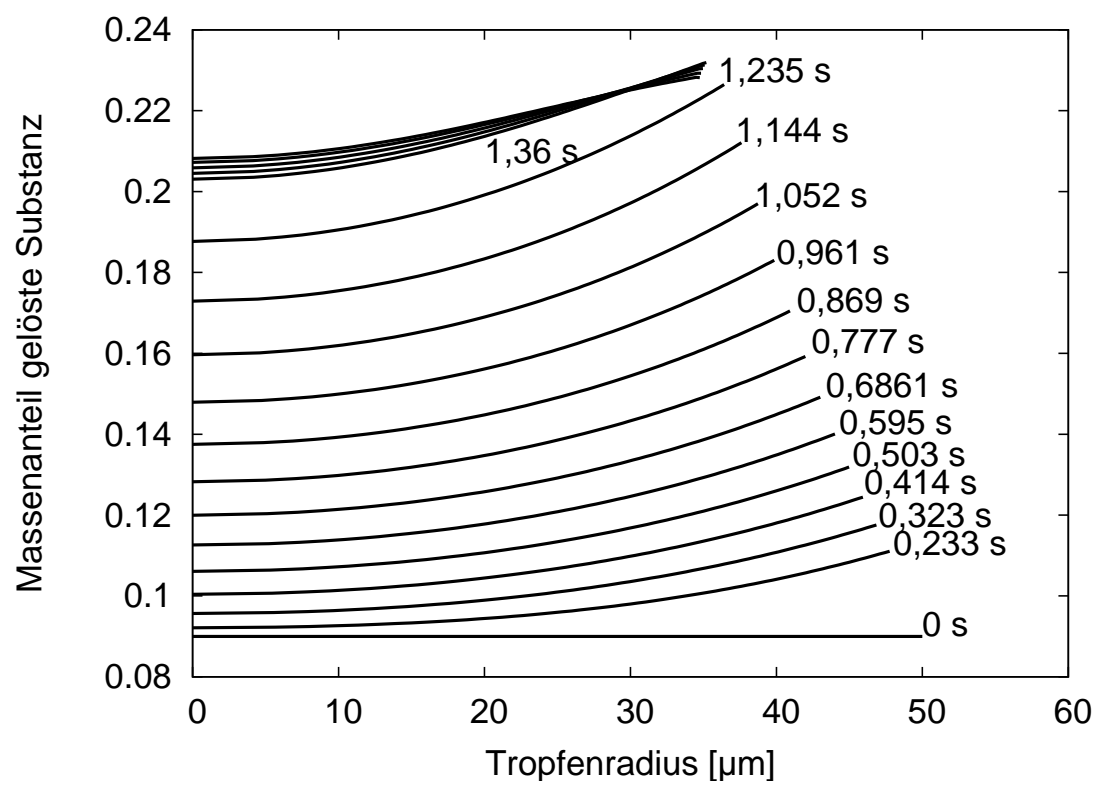

Abbildung 8.10: Zeitliche Änderung der radialen Verteilung des Massenanteils an $\mathrm{K}_{2} \mathrm{SO}_{4}$ in einem $\mathrm{K}_{2} \mathrm{SO}_{4}$-Lösungstropfen (Lufttemperatur $T_{U}=323 \mathrm{~K}$, übrige Parameter wie Abb. 8.8) 


\subsubsection{Einfluß der Trocknungsgastemperatur}

Im folgenden wird die Trocknungsgastemperatur variiert. Je geringer die Trocknungsgastemperatur ist, desto langsamer verdunstet der Tropfen. Aufgrund der langsameren Schrumpfung des Tropfens ist der Gradient des Massenanteils der gelösten Substanz geringer, weil mehr Zeit für den diffusiven Konzentrationsausgleich vorhanden ist. Daher bilden sich tendentiell mehr Partikel im Inneren des Tropfens, je langsamer der Tropfen schrumpft. Darüber hinaus ist zu erwarten, daß sich weniger Partikel bilden, weil die Übersättigung geringer ist. Brenn [27] hat eine Kennzahl G entwickelt, die eine Unterscheidung erlaubt, wann Vollpartikel und wann Hohlpartikel entstehen

$$
G=\frac{D_{\text {Tropfen }}}{D_{\text {Gas }}} \frac{\rho_{\text {Tropfen }}}{\rho_{\text {Gas }}} \frac{1}{\operatorname{Sh} \cdot \ln \left(1+B_{M}\right)} .
$$

Ist $\mathrm{G}<3,3$, so entstehen Hohlpartikel. Ford [24] hat für verschiedene Trocknungsparameter mit seinem Modell simuliert, ob Voll- oder Hohlpartikel entstehen. Die Dicke der Hülle am Ende des Trocknungsprozesses wird aus dem Endradius am Ende des 1. Trocknungsabschnitts und der Masse der gelösten Substanz berechnet, wobei für die Verteilung der gelösten Substanz eine Porosität von $74 \%$ angenommen wird. Dabei hat er festgestellt, daß bei Anfangstropfendurchmessern von 10 bis $30 \mu \mathrm{m}$ und geringen Trocknungsgastemperaturen von weniger als 333K Vollkugeln entstehen. Da bei niedrigen Temperaturen wegen der langsamen Verdunstung mehr Zeit für einen Konzentrationsausgleich vorhanden ist, wird die Sättigungsgrenze auch im Tropfeninneren erreicht, wodurch es dann gleichmäßig über den Tropfenradius verteilt zur Partikelbildung kommt. In Abb. 8.11 ist die Partikelanzahldichte am Ende des 1. Trocknungsabschnitts über dem Tropfenradius und der Partikelgröße dargestellt. Der Ausgangstropfendurchmesser liegt zwischen 10 und $80 \mu \mathrm{m}$. Die Trocknungsbedingungen liegen im Bereich von $\mathrm{G}>3,3$. In allen Abbildungen ist zu erkennen, daß sich auch im Tropfeninneren Partikel entwickelt haben. Da jedoch der zurückweichende Tropfenrand die Partikel aufsammelt, ist die Partikelanzahldichte am Tropfenrand deutlich höher als in der Tropfenmitte. Dies verdeutlicht auch Abb. 8.12, welche die Verteilung des Partikelvolumenanteils über den Tropfenradius zeigt. Der Partikelvolumenanteil ist am Tropfenrand deutlich größer als im Tropfeninneren. In dem Modell von Ford ist jedoch unklar, wie sich die Partikel am Tropfenrand verhalten, was einen Vergleich erschwert. 

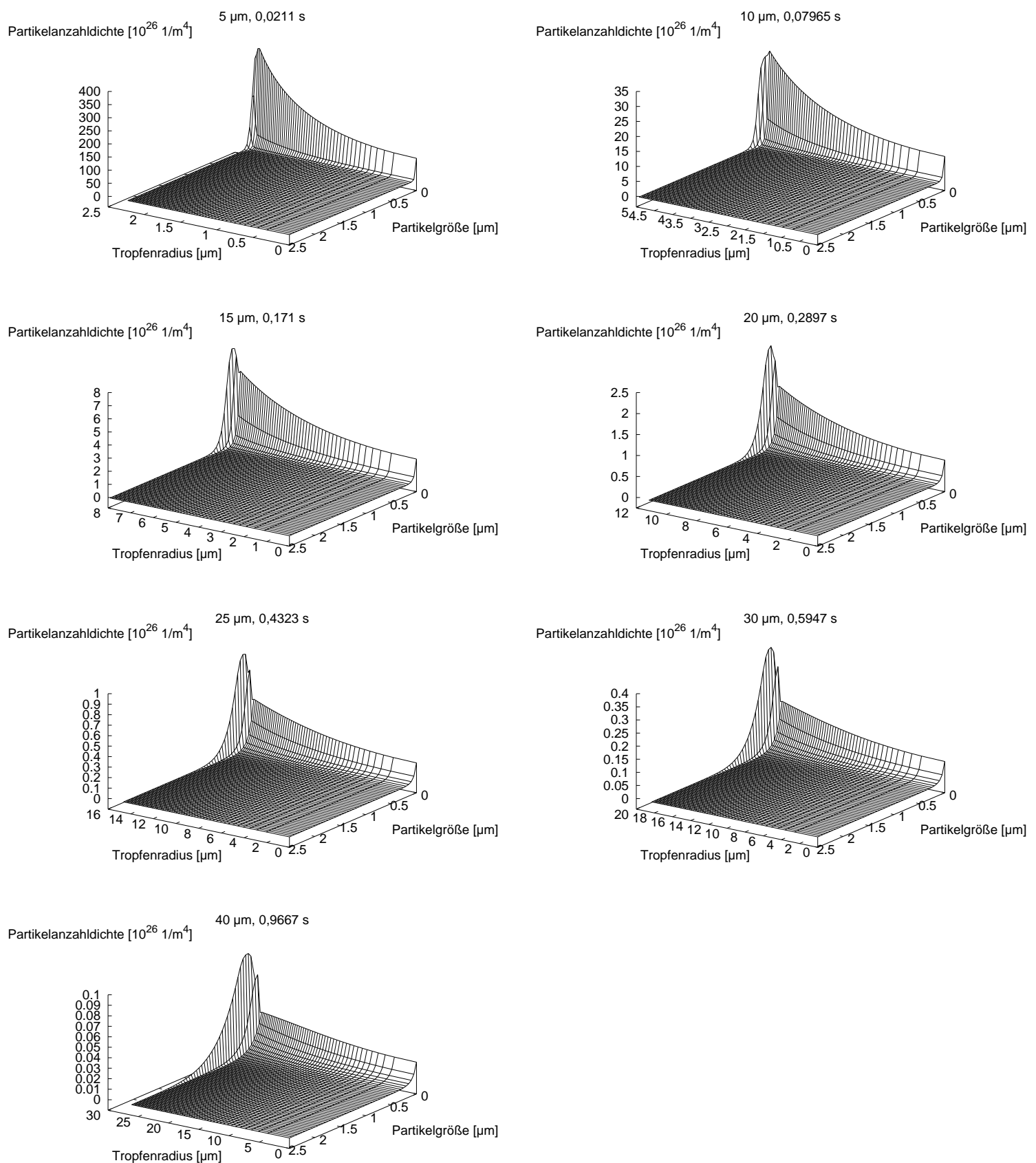

Abbildung 8.11: Partikelanzahldichte über dem Tropfenradius und der Partikelgröße am Ende des 1. Trocknungsabschnitts bei $\mathrm{K}_{2} \mathrm{SO}_{4}$-Lösungstropfen mit unterschiedlichem Anfangsdurchmesser bei einer Lufttemperatur von $50^{\circ} \mathrm{C}$

(Massenanteil der gelösten Substanz $w_{G}(t=0 s)=0,09$, Dichte der gelösten Substanz $\rho_{G}^{\prime}=2670 \mathrm{~kg} / \mathrm{m}^{3}$, Dichte des Lösungsmittels $\rho_{L}^{\prime}=1000 \mathrm{~kg} / \mathrm{m}^{3}$, Tropfentemperatur $T_{\text {Tropfen }}(t=0 s)=293 \mathrm{~K}$, Wärmekapazität $c_{p_{\text {Wasser }}}=4,183 \mathrm{~kJ} /(\mathrm{kgK})$, Wärmekapazität $c_{p_{G}}=0,92 \mathrm{~kJ} /(\mathrm{kgK})$, Wärmeleitfähigkeit $\lambda_{\text {Tropfen }}=0,637 \mathrm{~W} /\left(\mathrm{m}^{2} \mathrm{~K}\right)$, Wärmeleitfähigkeit $\lambda_{\text {Luft }}=0,03795 \mathrm{~W} /\left(\mathrm{m}^{2} \mathrm{~K}\right)$, Lufttemperatur $\left.T_{U}=323 \mathrm{~K}\right)$ 


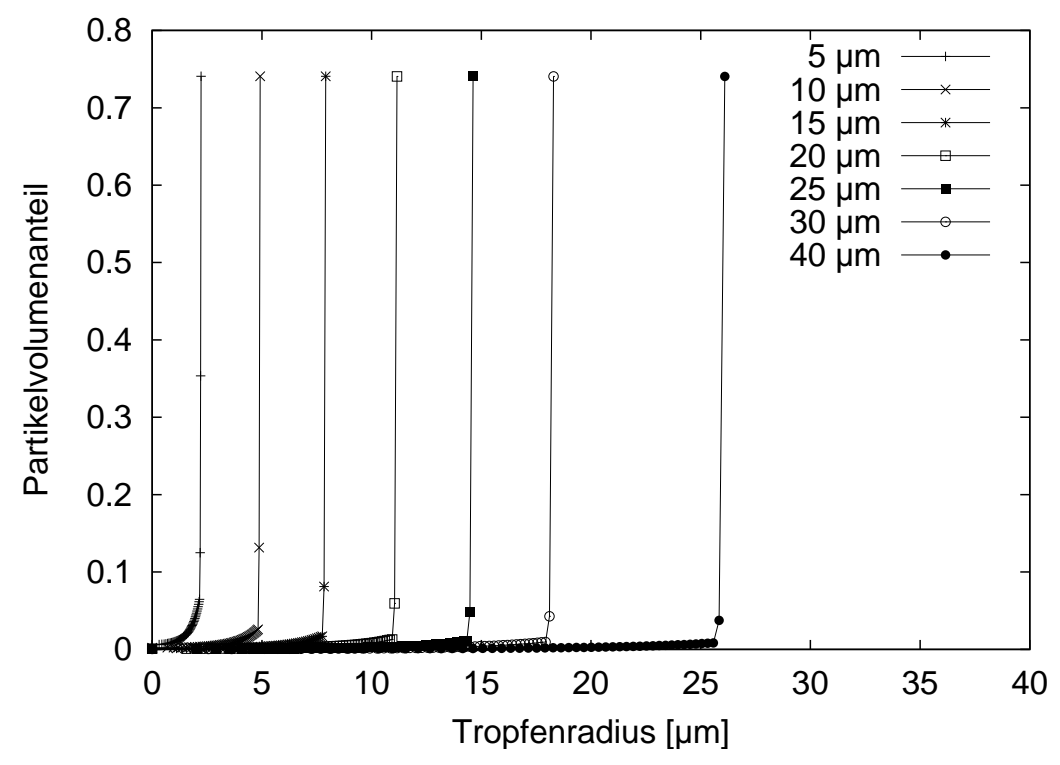

Abbildung 8.12: Partikelvolumenanteil in Abhängigkeit vom Tropfenradius in einem $\mathrm{K}_{2} \mathrm{SO}_{4^{-}}$ Lösungstropfen am Ende des 1. Trocknungsabschnitts bei unterschiedlichen Anfangsradien und einer Lufttemperatur von 323K (Parameter siehe Abb. 8.11)

In den Abb. 8.13 und 8.14 ist der zeitliche Übersättigungsverlauf am Tropfenrand für Ammoniumsulfat und Kaliumsulfat dargestellt. Auffällig ist, daß beim Kaliumsulfat mit steigender Temperatur deutlich höhere Übersättigungen im Tropfen erreicht werden, während beim Ammoniumsulfat der Unterschied nur gering ist. Dies läßst sich mit dem langsamen Kristallwachstum und der geringen Keimbildungsrate sowie der Größenabhängigkeit des Kristallwachstums bei der Kaliumsulfatkinetik erklären. Bei schneller Trocknung baut sich in kurzer Zeit eine hohe Übersättigung auf und es bilden sich zunächst viele kleine Partikel, die langsam wachsen, so daß die Übersättigung nur langsam abgebaut wird. Bei langsamer Trocknung baut sich die Übersättigung langsam auf und es entstehen wenige kleine Partikel, die mehr Zeit zum Wachsen haben und mit zunehmender Größe einen immer größeren Teil der Übersättigung abbauen. Wegen der Temperaturabhängigkeit der Löslichkeit nimmt beim Kaliumsulfat darüber hinaus zunächst die Übersättigung aufgrund der zunehmenden Löslichkeit ab, bis der Tropfen die Kühlgrenztemperatur erreicht hat. Da bei der Ammoniumsulfatkinetik die Wachstumsrate der Partikel nicht größenabhängig ist, wachsen die kleinen Partikel ebenso schnell wie die großen, wodurch auch viele kleine Partikel eine großen Teil der Übersättigung abbauen. Da der Tropfenrand entscheidend für die Hüllenbildung ist, wird in den Abb. 8.15 und 8.16 die Partikelvolumenverteilung am Tropfenrand am Ende des 1. Trocknungsabschnitts dargestellt. Die Partikelanzahldichtefunktionen werden dabei gemäß

$$
n^{*}=\frac{\pi}{6} \cdot n L^{3}
$$




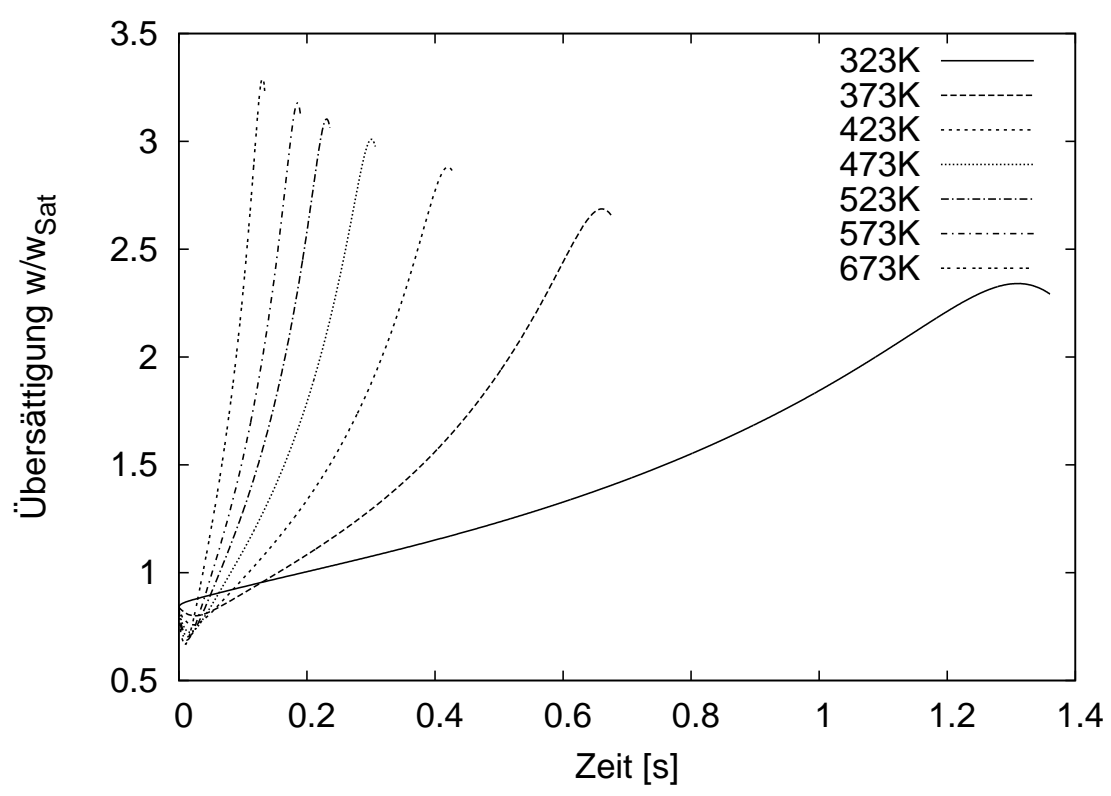

Abbildung 8.13: Zeitlicher Verlauf der Übersättigung w/w $\mathrm{w}_{\text {Sat }}$ am Tropfenrand für verschiedene Trocknungsgastemperaturen bei einem $\mathrm{K}_{2} \mathrm{SO}_{4}$-Lösungstropfen

(Massenanteil der gelösten Substanz $w_{G}(t=0 s)=0,09$, Dichte der gelösten Substanz $\rho_{G}^{\prime}=2670 \mathrm{~kg} / \mathrm{m}^{3}$, Dichte des Lösungsmittels $\rho_{L}^{\prime}=1000 \mathrm{~kg} / \mathrm{m}^{3}$, Tropfentemperatur $T_{\text {Tropfen }}(t=0 s)=293 \mathrm{~K}$, Wärmekapazität $c_{p_{\text {Wasser }}}=4,183 \mathrm{~kJ} /(\mathrm{kgK})$, Wärmekapazität $c_{p_{G}}=0,92 \mathrm{~kJ} /(\mathrm{kgK})$, Wärmeleitfähigkeit $\lambda_{\text {Tropfen }}=0,637 \mathrm{~W} /\left(\mathrm{m}^{2} \mathrm{~K}\right)$, Wärmeleitfähigkeit $\left.\lambda_{\text {Luft }}=0,03795 \mathrm{~W} /\left(\mathrm{m}^{2} \mathrm{~K}\right)\right)$

transformiert. Die volumenbasierte Verteilungsfunktion $n^{*}$ ist mit dem Volumen einer Tropfenschale normiert, wodurch die Fläche unterhalb der Verteilungsfunktionen dem Partikelvolumenanteil entspricht. Da der Partikelvolumenanteil am Ende des 1. Trocknungsabschnitts $74 \%$ beträgt, hat die Fläche unterhalb der Verteilungen eine Größe von 0,74. Es zeigt sich, daß die Breite der Partikelgrößenverteilung am Tropfenrand mit zunehmender Temperatur abnimmt. Dies läßt sich mit den in den Abb. 8.13 und 8.14 dargestellten Übersättigungsverläufen erklären. Es ist zu erkennen, daß der Übersättigungsverlauf bei hohen Temperaturen steiler ist als bei niedrigen. Durch den flacheren Verlauf der Übersättigung bei langsamer Trocknung entstehen weniger Partikel und die entstehende Übersättigung wird durch das Wachstum der Partikel aufgebraucht. Die Abb. 8.17 und 8.18 zeigen den Endradius und die mittlere Partikelgröße von $\mathrm{K}_{2} \mathrm{SO}_{4}$ - und $\left(\mathrm{NH}_{4}\right)_{2} \mathrm{SO}_{4}$-Lösungstropfen in Abhängigkeit von der Trocknungsgastemperatur, wobei der Ausgangsradius der Tropfen jeweils $50 \mu \mathrm{m}$ beträgt. Im Falle von Ammoniumsulfat ist deutlich zu sehen, daß der Endradius mit zunehmender Temperatur größer wird, während im Falle von Kaliumsulfat der Endradius bei einer Temperatur von 423K ein Minimum erreicht und anschließend wieder ansteigt. Beim Ammoniumsulfat wird bei 


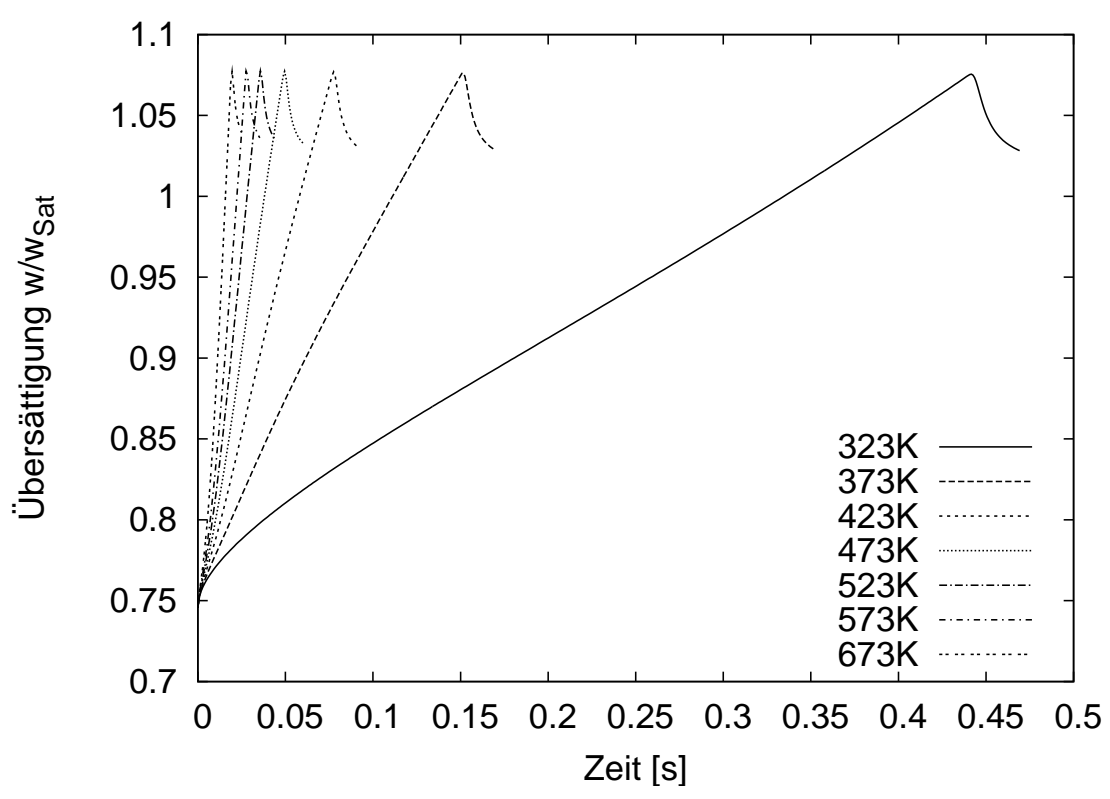

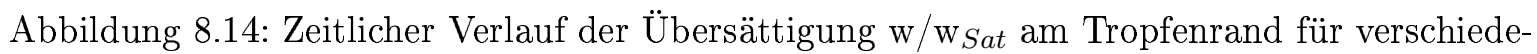
ne Trocknungsgastemperaturen bei einem $\left(\mathrm{NH}_{4}\right)_{2} \mathrm{SO}_{4}$-Lösungstropfen

(Massenanteil der gelösten Substanz $w_{G}(t=0 s)=0,34$, Dichte der gelösten Substanz $\rho_{G}^{\prime}=$ $1770 \mathrm{~kg} / \mathrm{m}^{3}$, Dichte des Lösungsmittels $\rho_{L}^{\prime}=1000 \mathrm{~kg} / \mathrm{m}^{3}$, TropfentemperaturT $T_{\text {Tropfen }}(t=$ $0 s)=293 K$, Wärmekapazität $c_{p_{\text {Wasser }}}=4,183 k J /(k g K)$, Wärmekapazität $c_{p_{G}}=$ $0,92 \mathrm{~kJ} /(\mathrm{kgK})$, Wärmeleitfähigkeit $\lambda_{\text {Tropfen }}=0,637 \mathrm{~W} /\left(\mathrm{m}^{2} \mathrm{~K}\right)$, Wärmeleitfähigkeit $\lambda_{\text {Luft }}=$ $\left.0,03795 W /\left(m^{2} K\right)\right)$ 
höheren Temperaturen die Sättigungsgrenze schneller überschritten, wodurch die Kristallisation zu einem früheren Zeitpunkt beginnt und aufgrund des steileren Übersättigungsverlaufs schneller abläuft. Dadurch wird der kritische Feststoffvolumenanteil am Tropfenrand schneller erreicht, was einen größeren Endradius am Ende des 1. Trocknungsabschnitts zur Folge hat. Das Endradiusminimum bei der Kaliumsulfatlösung ist eine Folge der Größenabhängigkeit des Kristallwachstums. Durch den Übersättigungsverlauf ergibt sich ein bestimmtes Verhältnis von Keimbildung zu Kristallwachstum. Im Falle des Kaliumsulfatlösungstropfens nimmt das Wachstum der Partikel durch den langsameren Trocknungsverlauf so stark zu, daß bereits bei einem größeren Endradius der Volumenanteil von $74 \%$ erreicht wird. Bei beiden Lösungen nimmt die mittlere Partikelgröße mit zunehmender Temperatur ab, weil der Übersättigungsverlauf mit zunehmender Temperatur steiler wird und somit mehr Keime entstehen.

Zusammenfassend kann also der Schluß gezogen werden, daß bei langsamer Trocknung tendentiell Vollpartikel entstehen, die aus weniger und größeren Kristallen bestehen, während bei schneller Trocknung Hohlpartikel entstehen, die aus vielen kleinen Kristallen bestehen. Bei Ammoniumsulfatlösungstropfen ist der Endradius umso größer, je größer die Trocknungsgeschwindigkeit ist. Dies bestätigen auch experimentelle Untersuchungen von Walton und Mumford für Kochsalz [65]. Bei den Simulationsrechnungen für einen Kaliumsulfatlösungstropfen läßst sich überraschenderweise bei $473 \mathrm{~K}$ ein Minimum des Endradius beobachten. 


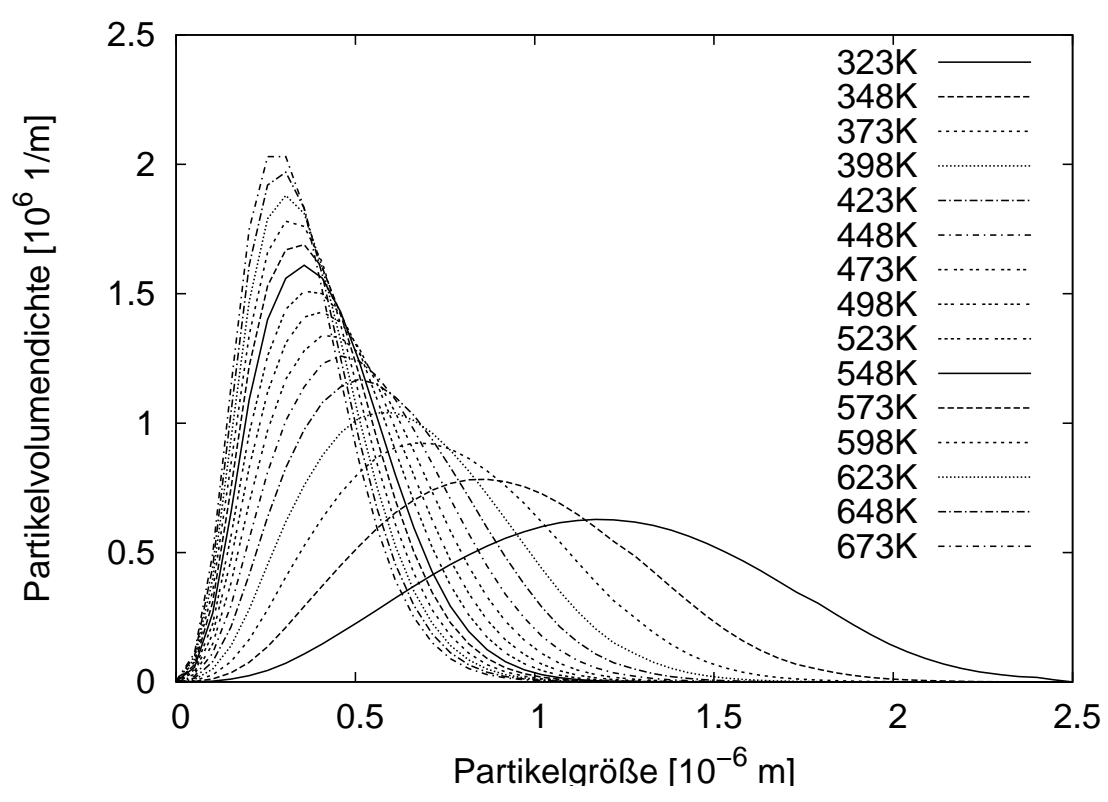

Abbildung 8.15: Partikelvolumendichteverteilung am Tropfenrand in Abhängigkeit von der Partikelgröße am Ende des 1. Trocknungsabschnitts bei einem $\mathrm{K}_{2} \mathrm{SO}_{4}$-Lösungstropfen für unterschiedliche Trocknungsgastemperaturen (Parameter siehe Abb. 8.13)

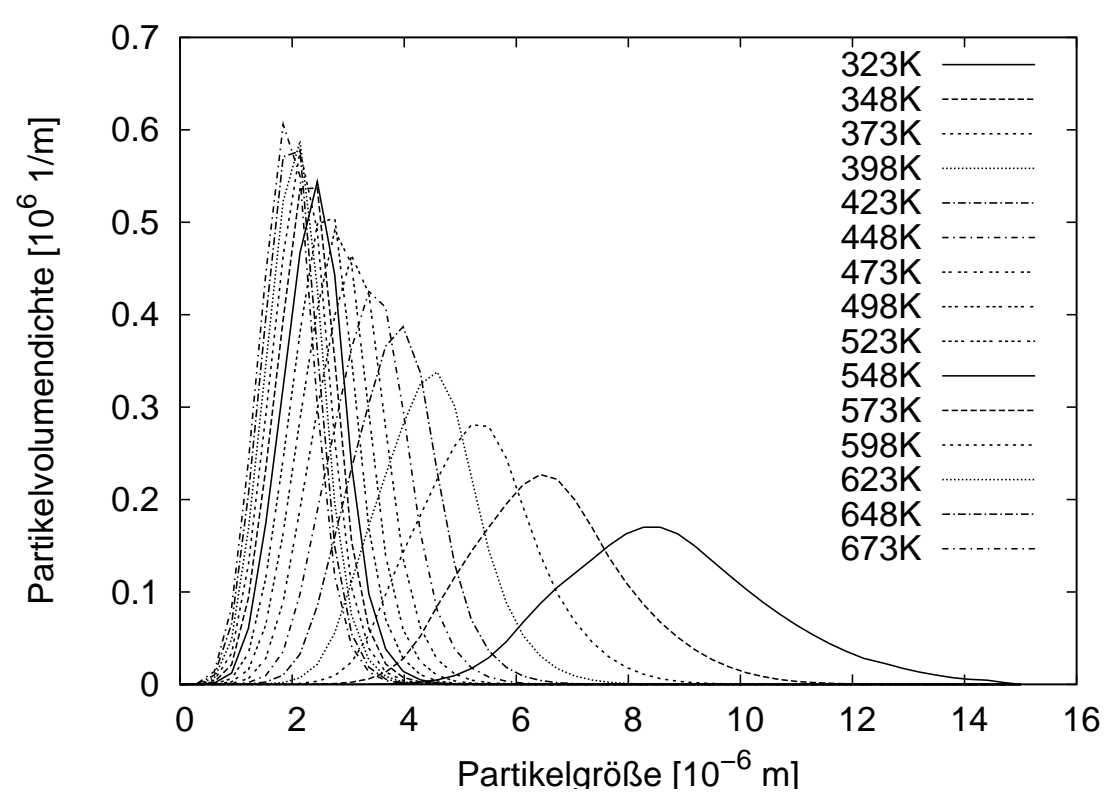

Abbildung 8.16: Partikelvolumendichteverteilung am Tropfenrand in Abhängigkeit von der Partikelgröße am Ende des 1. Trocknungsabschnitts bei einem $\left(\mathrm{NH}_{4}\right)_{2} \mathrm{SO}_{4}$-Lösungstropfen für unterschiedliche Trocknungsgastemperaturen (Parameter siehe Abb. 8.14) 


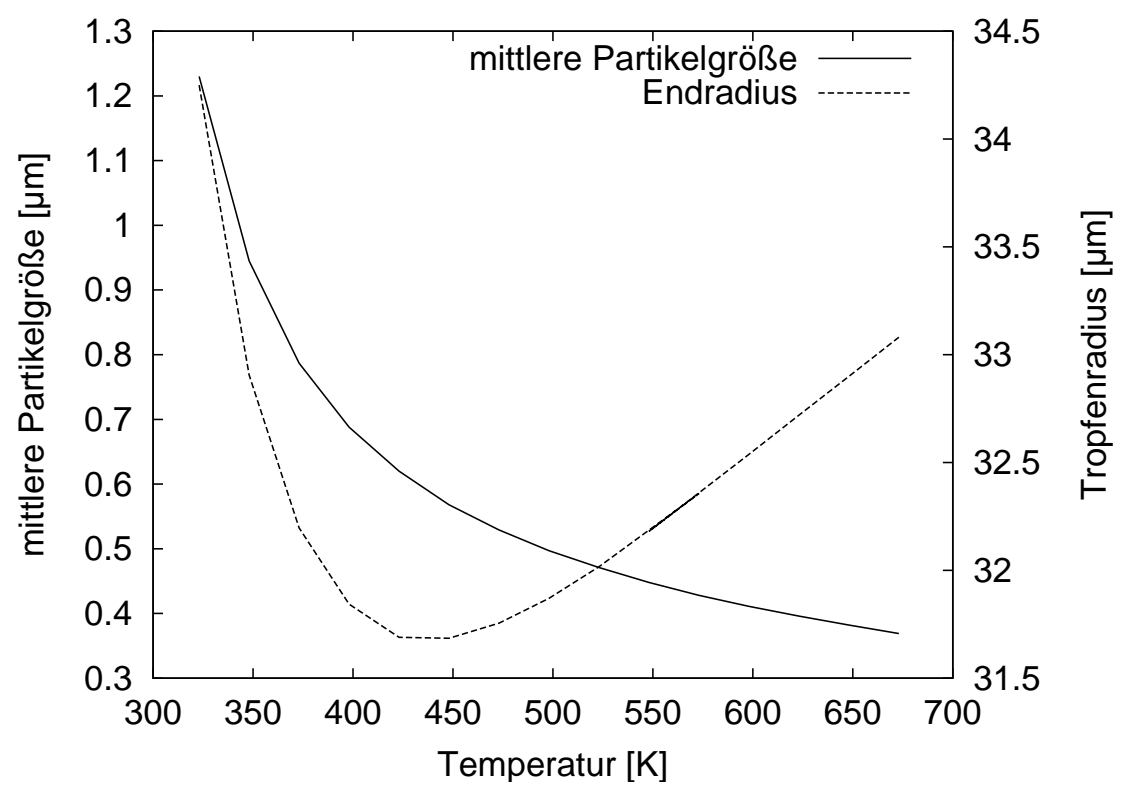

Abbildung 8.17: Endradius und mittlere Partikelgröße eines $\mathrm{K}_{2} \mathrm{SO}_{4}$-Lösungstropfens am Ende des 1. Trocknungsabschnitts für verschiedene Trocknungsgastemperaturen (Parameter siehe Abb. 8.13)

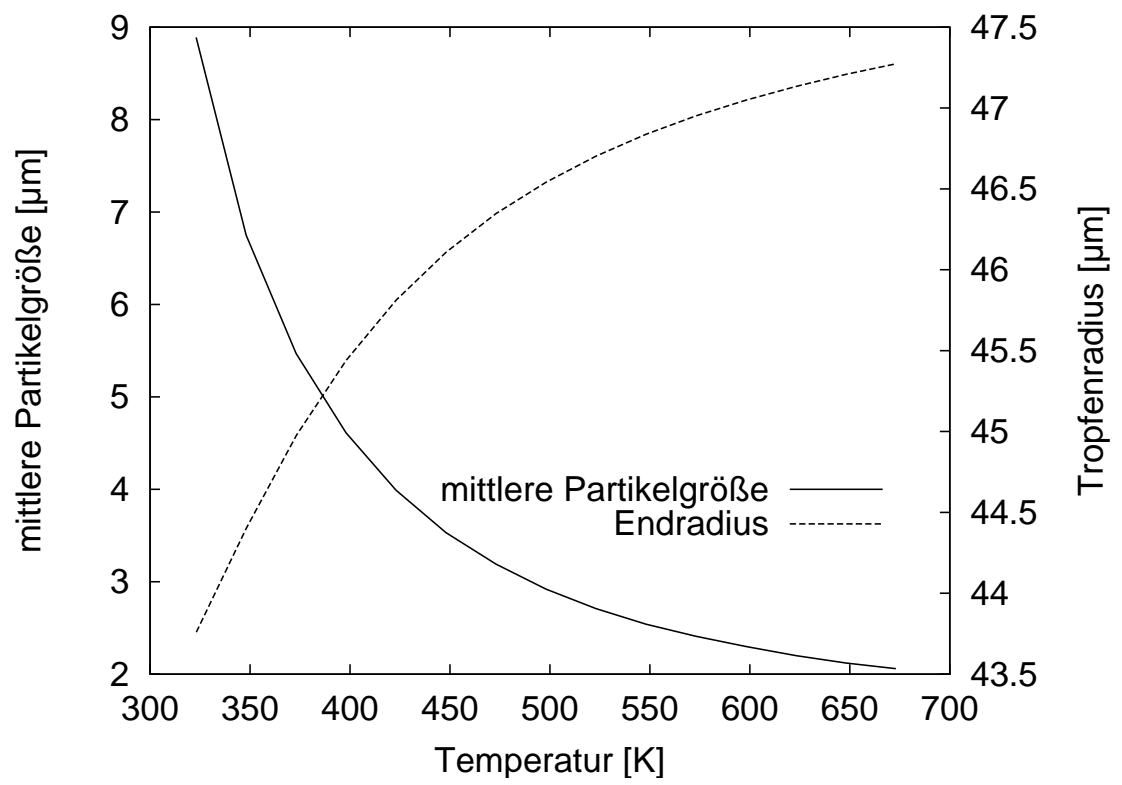

Abbildung 8.18: Endradius und mittlere Partikelgröße eines $\left(\mathrm{NH}_{4}\right)_{2} \mathrm{SO}_{4}$-Lösungstropfens am Ende des 1. Trocknungsabschnitts für verschiedene Trocknungsgastemperaturen (Parameter siehe Abb. 8.14) 


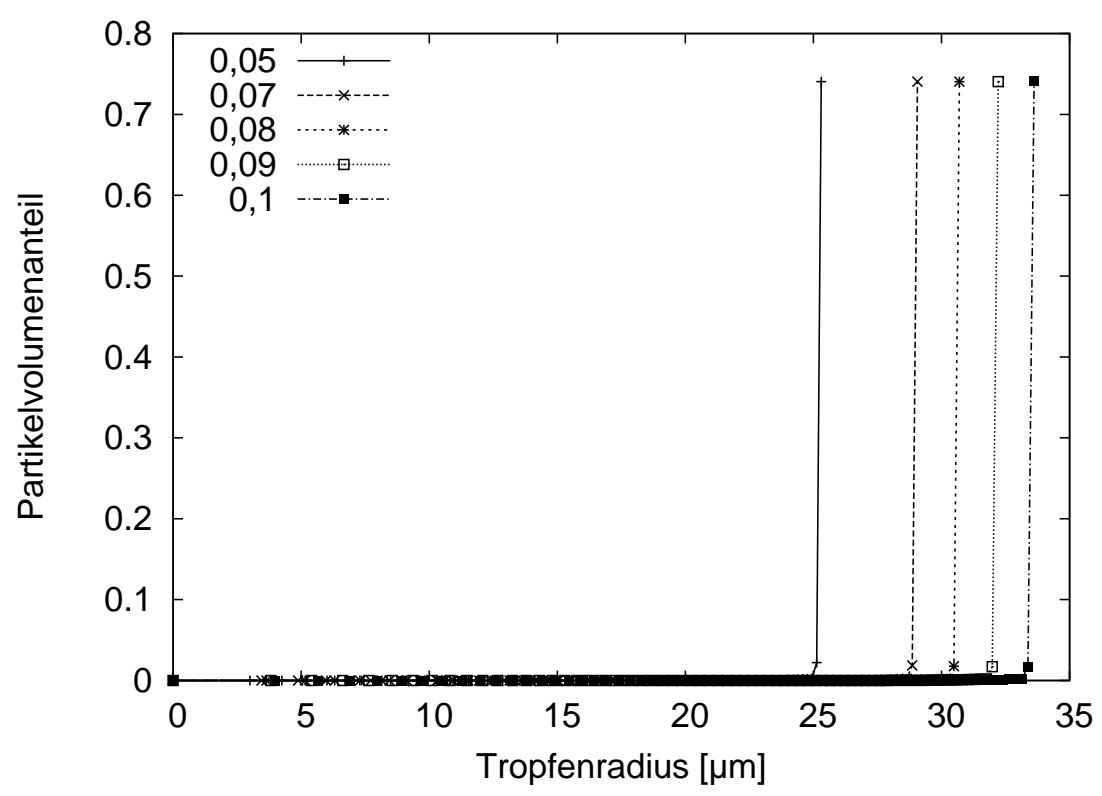

Abbildung 8.19: Partikelvolumenanteil über dem Tropfenradius am Ende des 1. Trocknungsabschnitts bei einem $\mathrm{K}_{2} \mathrm{SO}_{4}$-Lösungstropfen für verschiedene Anfangsmassenanteile an $\mathrm{K}_{2} \mathrm{SO}_{4}$ bei einer Trocknungsgastemperatur von 473K und einer Tropfentemperatur von 293K (Parameter siehe Abb. 8.1)

\subsubsection{Einfluß der Lösungskonzentration}

Bei der Sprühtrocknung ist es vom energetischen Standpunkt aus betrachtet am günstigsten, wenn möglichst hoch konzentrierte Lösungen versprüht werden, weil weniger Wasser verdunstet werden muß. Da die Löslichkeit der Salze temperaturabhängig ist, kann durch Erhitzen der Lösung im allgemeinen eine weitere Erhöhung des Salzmassenanteils erreicht werden. Abb. 8.19 zeigt die Verteilung des Partikelvolumenanteils in einem $\mathrm{K}_{2} \mathrm{SO}_{4}$-Lösungstropfen am Ende des 1. Trocknungsabschnitts über dem Tropfenradius. Es ist zu erkennen, daß Partikel mit einem höheren Startmassenanteil einen größeren Endradius haben, weil die Partikelbildung früher beginnt und somit der Partikelvolumenanteil am Tropfenrand früher den kritischen Wert erreicht. Durch das Aufsammeln der Partikel am Tropfenrand kommt es auch bei einem höheren Massenanteil zu einer erhöhten Partikelkonzentration am Tropfenrand. Bei einem hohen Ausgangsmassenanteil bilden sich in dem $\mathrm{K}_{2} \mathrm{SO}_{4}$-Lösungstropfen am Tropfenrand eher große Partikel, während sich bei einem niedrigen Ausgangsmassenanteil kleine Partikel bilden (Abb. 8.20). Im Falle des $\left(\mathrm{NH}_{4}\right)_{2} \mathrm{SO}_{4}$-Lösungstropfens bilden sich bei einer hohen Anfangskonzentration eher kleine Kristalle, während bei niedrigen Anfangskonzentrationen große enstehen. Die Abhängigkeit der Partikelgröße von der Anfangskonzentration an $\mathrm{K}_{2} \mathrm{SO}_{4}$ läßt sich darauf zurückführen, daß bei einem niedrigen Massenanteil die Sättigungsgrenze spä- 


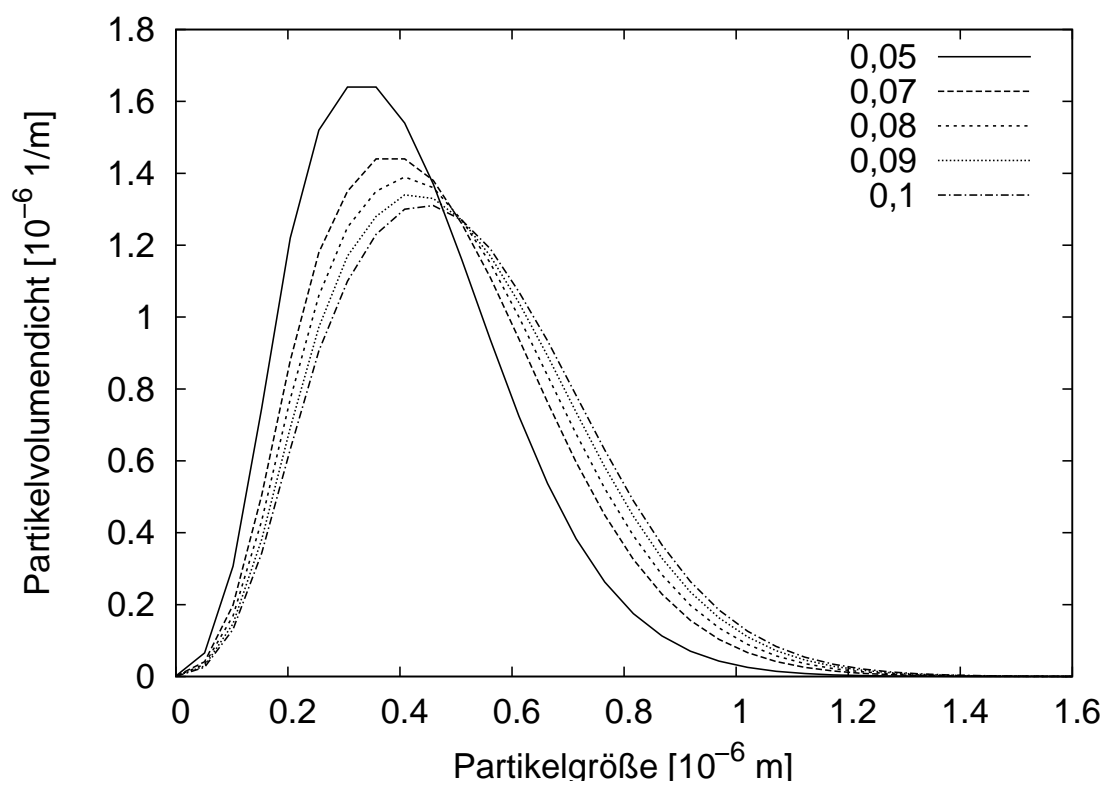

Abbildung 8.20: Partikelanzahldichteverteilung in Abhängigkeit von der Partikelgröße am Tropfenrand am Ende des 1. Trocknungsabschnitts bei einem $\mathrm{K}_{2} \mathrm{SO}_{4}$-Lösungstropfen für verschiedene Anfangsmassenanteile an $\mathrm{K}_{2} \mathrm{SO}_{4}$ bei einer Trocknungsgastemperatur von $473 \mathrm{~K}$ und einer Tropfentemperatur von 293K (Parameter siehe Abb. 8.1)

ter erreicht wird. Zu dem Zeitpunkt, an dem die Kristallisation beginnt, ist der Verlauf der Übersättigung steiler, weil der Tropfen mit abnehmendem Radius schneller schrumpft (Abb. 8.22). Durch den steileren Verlauf der Übersättigung entstehen mehr kleine Partikel als bei einem hohen Anfangsmassenanteil. Bei dem $\left(\mathrm{NH}_{4}\right)_{2} \mathrm{SO}_{4}$-Lösungstropfen hingegen ist der Übersättigungsverlauf umso steiler, je höher der Anfangsmassenanteil ist (Abb. 8.23). In experimentellen Untersuchungen von [65] ist bei Kochsalz ein Verhalten entsprechend dem vom Ammoniumsulfat beobachtet worden. 


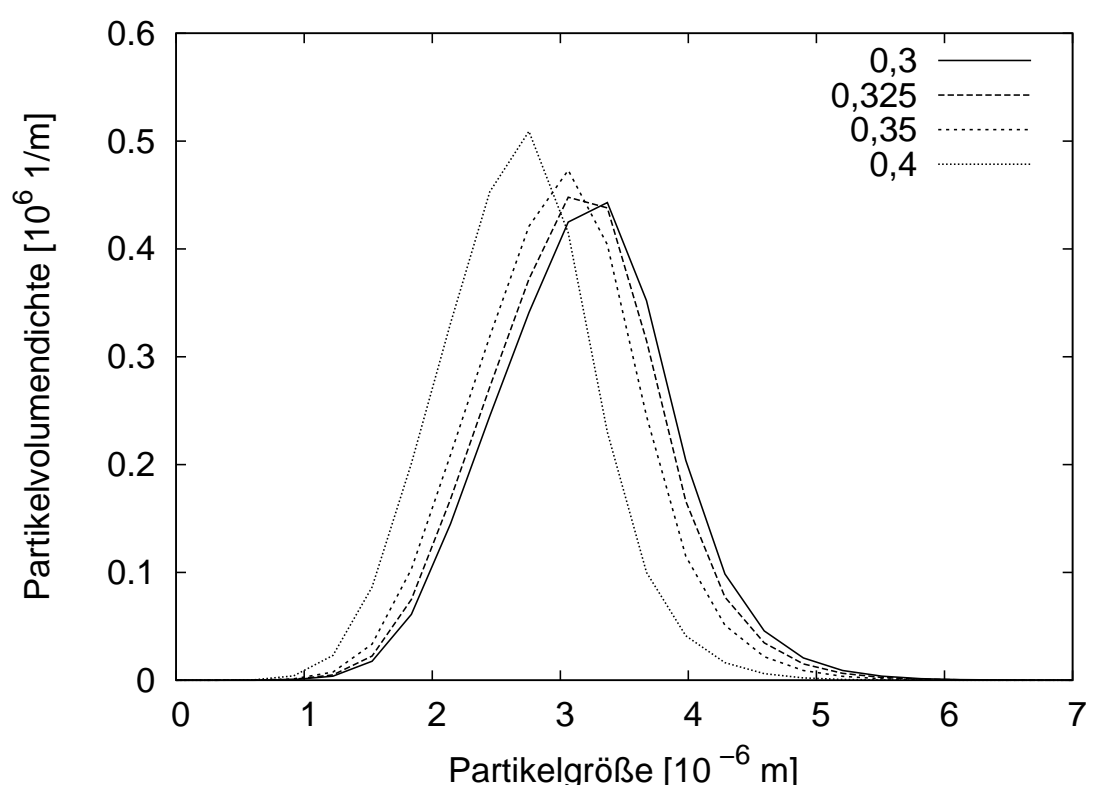

Abbildung 8.21: Partikelanzahldichteverteilung in Abhängigkeit von der Partikelgröße am Tropfenrand am Ende des 1. Trocknungsabschnitts bei einem $\left(\mathrm{NH}_{4}\right)_{2} \mathrm{SO}_{4}$-Lösungstropfen für verschiedene Anfangsmassenanteile an $\left(\mathrm{NH}_{4}\right)_{2} \mathrm{SO}_{4}$ bei einer Trocknungsgastemperatur von 473K und einer Tropfentemperatur von 293K (Parameter siehe Abb. 8.14)

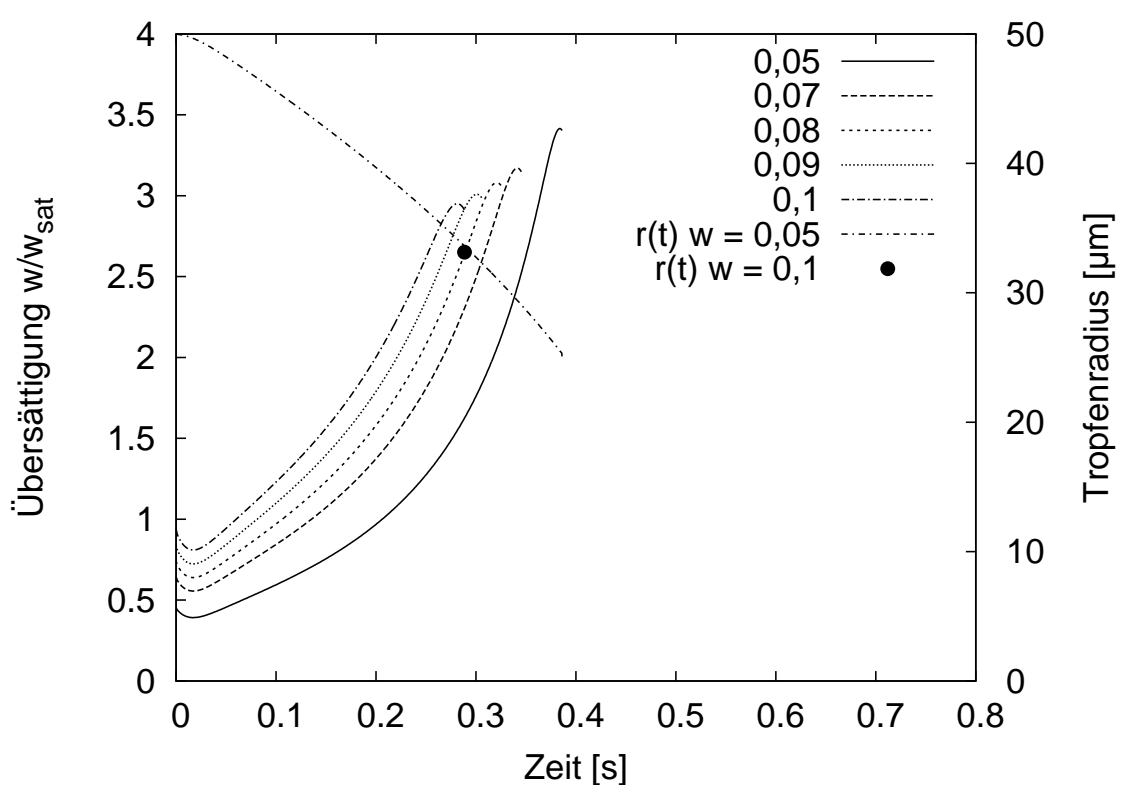

Abbildung 8.22: Zeitlicher Verlauf der Übersättigung w/w $\mathrm{w}_{\text {Sat }}$ am Tropfenrand am Ende des 1. Trocknungsabschnitts bei einem $\mathrm{K}_{2} \mathrm{SO}_{4}$-Lösungstropfen für verschiedene Anfangsmassenanteile an $\mathrm{K}_{2} \mathrm{SO}_{4}$ bei einer Trocknungsgastemperatur von $473 \mathrm{~K}$ und einer Tropfentemperatur von 293K. Zeitlicher Verlauf des Tropfenradius für zwei Anfangsmassenanteile (Parameter siehe Abb. 8.1) 


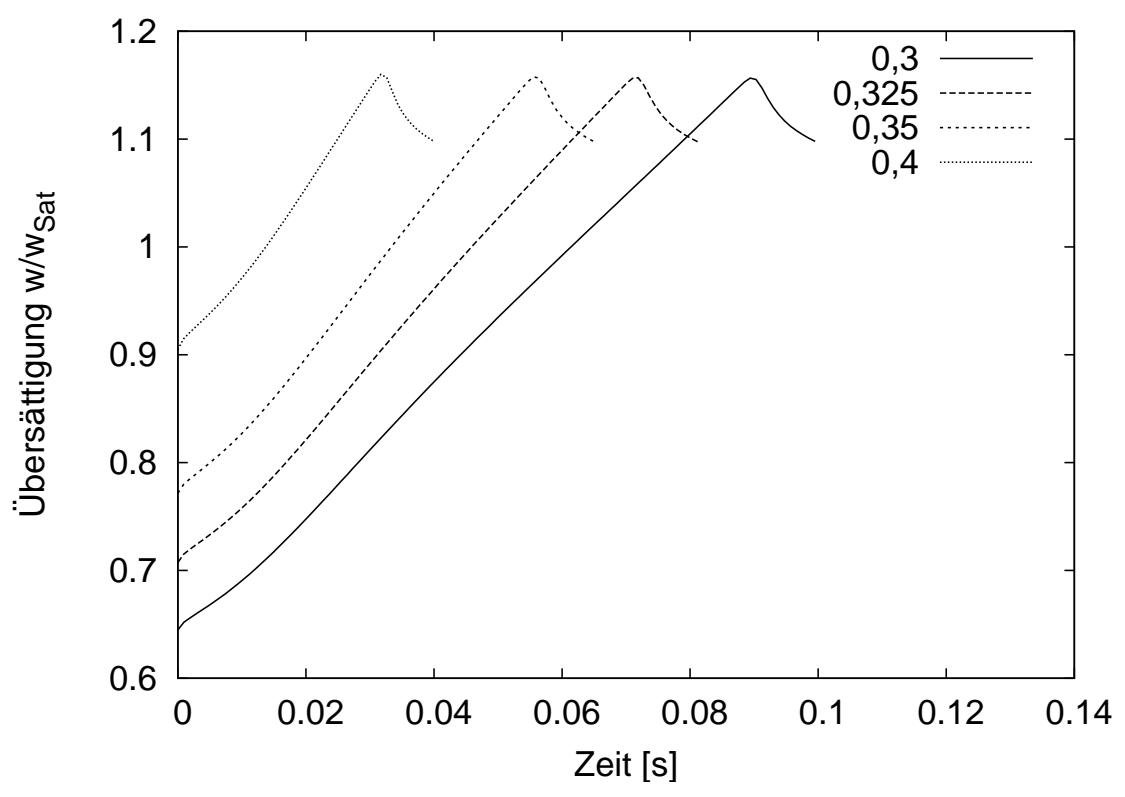

Abbildung 8.23: Zeitlicher Verlauf der Übersättigung w/w Sat am Tropfenrand am Ende des 1. Trocknungsabschnitts bei einem $\left(\mathrm{NH}_{4}\right)_{2} \mathrm{SO}_{4}$-Lösungstropfen für verschiedene Anfangsmassenanteile an $\left(\mathrm{NH}_{4}\right)_{2} \mathrm{SO}_{4}$ bei einer Trocknungsgastemperatur von $473 \mathrm{~K}$ und einer Tropfentemperatur von 293K (Parameter siehe Abb. 8.14) 


\subsection{Simulation mit gleichzeitiger Bilanzierung des Trocknungs- gases}

Bei der Sprühtrocknung verändert sich mit fortschreitender Trocknung auch der Zustand des Trocknungsgases. Unter der Annahme, daß ein Tröpfchenschwarm immer mit dem gleichen Gasvolumen Wärme und Masse austauscht, kann der reale Prozeß auch ohne die Verwendung von CFD-Software simuliert werden (Abb. 8.24). Die Dampfmassenänderung im Trocknungsgas ergibt sich aus der verdunsteten Lösungsmittelmenge zu

$$
\frac{d m_{\text {Dampf }}}{d t}=4 \pi r_{\text {Tropfen }}^{2} \dot{m}_{\text {Dampf }} \cdot \text { Tropfenzahl } .
$$

Der Dampfpartialdruck zu jedem Zeitpunkt ergibt sich dann aus

$$
p_{D}=\frac{\frac{m_{\text {Dampf }}}{m_{\text {trockene Luft }}} \frac{M_{\text {Trocknungsgas }}}{M_{\text {Dampf }}}}{1+\frac{m_{\text {Wasser }}}{m_{\text {trockene Luft }}} \frac{M_{\text {Trocknungsgas }}}{M_{\text {Dampf }}}} .
$$

Die Temperaturänderung des Trocknungsgases berechnet sich aus der Wärme, die zur Aufheizung der Tropfen verwendet wird. Die Verdampfungsenthalpie wird ebenfalls dem Trocknungsgas entzogen, diesem jedoch in Form von Dampf wieder zugeführt. Daher erscheint die Verdampfungsenthalpie nicht in der Gleichung.

$$
\frac{d T}{d t}=\frac{-4 \pi r_{\text {Tropfen }}^{2} \alpha\left(\left(T_{\text {Gas }}-T_{\text {Tropfen }}\right)\right.}{m_{\text {trockene Luft }} c_{p}} \cdot \text { Tropfenzahl }
$$

Abb. 8.25 zeigt den Temperaturverlauf und den Verlauf des Dampfpartialdrucks in der Luft für Anfangstemperaturen der Luft von $473 \mathrm{~K}$ und 673K. Im Falle der Anfangstemperatur der Luft von 473K wird zusätzlich der Verlauf des Sättigungsgrads der Trocknungsluft dargestellt. Der Tropfen heizt sich zunächst auf Kühlgrenztemperatur auf. Durch die Aufheizung der Wassertröpfchen kühlt sich die Luft jedoch ab und der Dampfpartialdruck nimmt zu. Da wegen

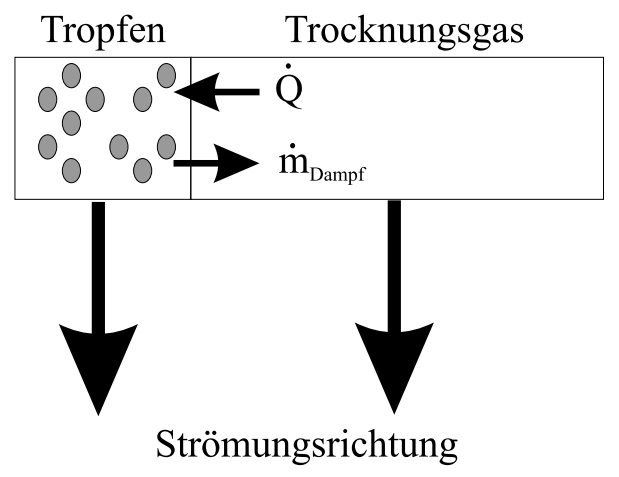

Abbildung 8.24: Die Tröpfchen tauschen immer mit dem gleichen Gasvolumen Wärme und Masse aus 

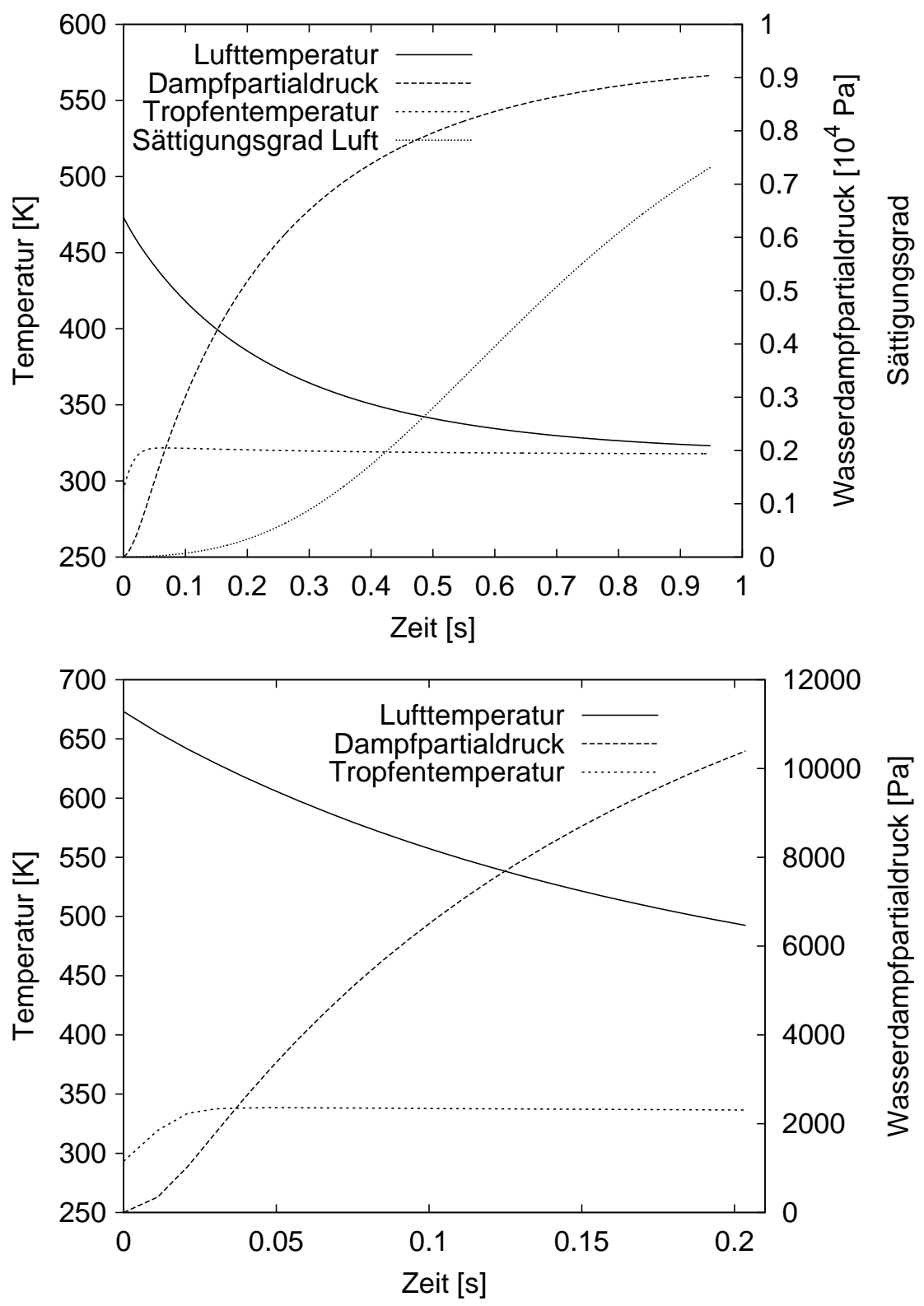

Abbildung 8.25: Zeitlicher Verlauf der Trocknungsgastemperatur und des Dampfpartialdrucks bei einer Masse des Tröpfchennebels von $5 \mathrm{~kg}$ und einer Luftmasse von $50 \mathrm{~kg}$ und einer Anfangslufttemperatur von 473K (oben) und 673K (unten) (Massenanteil der gelösten Substanz $w_{G}(t=0 s)=0,09$, Dichte der gelösten Substanz $\rho_{G}^{\prime}=2670 \mathrm{~kg} / \mathrm{m}^{3}$, Dichte des Lösungsmittels $\rho_{L}^{\prime}=1000 \mathrm{~kg} / \mathrm{m}^{3}$, Tropfentemperatur $T_{\text {Tropfen }}(t=0 \mathrm{~s})=293 \mathrm{~K}$, Wärmekapazität $c_{p_{\text {Wasser }}}=4,183 \mathrm{~kJ} /(\mathrm{kgK})$, Wärmekapazität $c_{p_{G}}=0,92 \mathrm{~kJ} /(\mathrm{kgK})$, Wärmeleitfähigkeit $\lambda_{\text {Tropfen }}=0,637 \mathrm{~W} /\left(\mathrm{m}^{2} K\right)$, Wärmeleitfähigkeit $\lambda_{\text {Luft }}=0,03795 \mathrm{~W} /\left(\mathrm{m}^{2} K\right)$, Wärmekapazität $c_{p_{\text {Luft }}}=1 \mathrm{~kJ} /(\mathrm{kgK})$, Dichte der Luft $\left.\rho_{\text {Luft }}^{\prime}=1 \mathrm{~kg} / \mathrm{m}^{3}\right)$ 
des hohen Partialdrucks die Verdunstung stark verlangsamt ist und die Kühlgrenztemperatur auch von der Partialdruckdifferenz abhängt, kühlt sich der Tropfen jedoch nicht mehr so stark ab. Bei der niedrigen Lufttemperatur von $473 \mathrm{~K}$ ist darüber hinaus zu sehen, daß die Luft einen hohen Sättigungsgrad erreicht. Durch die Verlangsamung des Trocknungsprozesses verändert sich auch die Partikelmorphologie, wie Abb. 8.26 zeigt, weil der Übersättigungsverlauf flacher ist und mehr Zeit für einen radialen Ausgleich der Verteilung des Massenanteils der gelösten Substanz vorhanden ist.

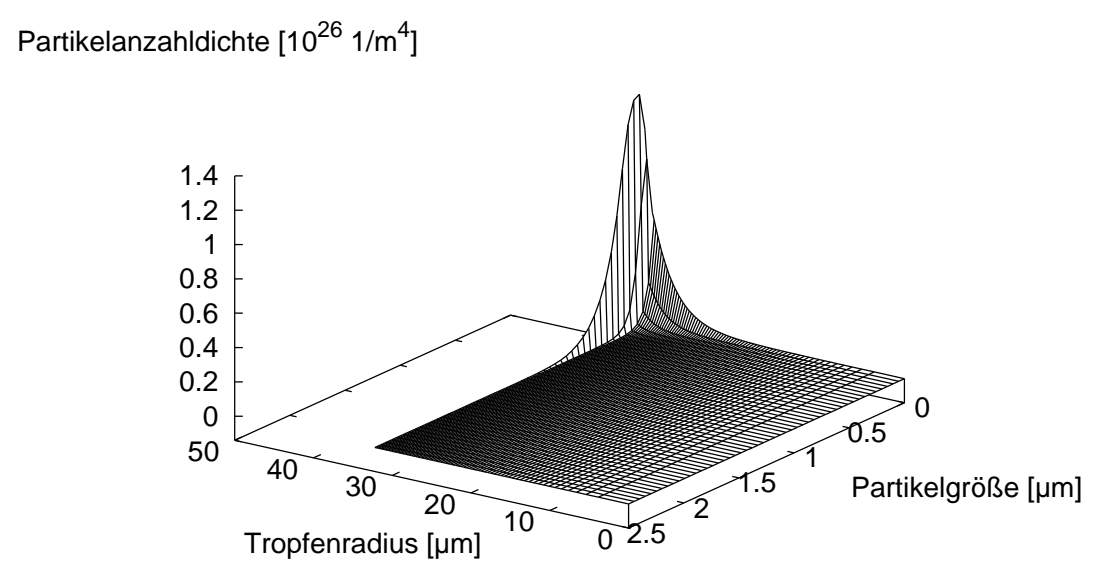

$$
\text { Partikelanzahldichte }\left[10^{26} 1 / \mathrm{m}^{4}\right]
$$

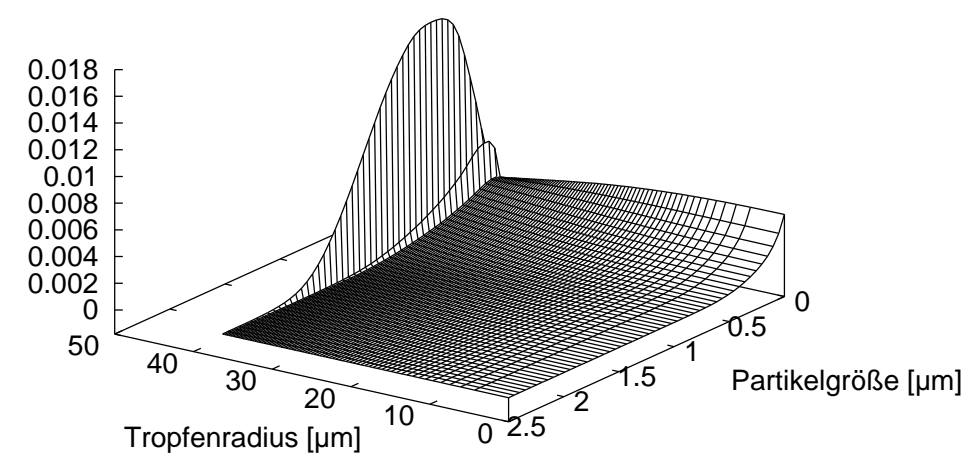

Abbildung 8.26: Partikelanzahldichteverteilung in einem $\mathrm{K}_{2} \mathrm{SO}_{4}$-Lösungstropfen am Ende des 1. Trocknungsabschnitts ohne (oben) und mit (unten) Berücksichtigung des Zustands der Luft bei einer Anfangslufttemperatur von 473K, wobei im Bild unten $5 \mathrm{~kg}$ Flüssigkeit in $50 \mathrm{~kg}$ Luft versprüht worden sind (Parameter siehe Abb. 8.25) 


\subsection{Einfluß der Diskretisierung auf die Partikelanzahldichtever- teilung}

Bei dem hier gewählten Modell nimmt eine Zelle nur so lange von innen kommende Partikel auf, bis ein bestimmter Volumenanteil erreicht ist. Anschließend nimmt der Volumenanteil in dieser Zelle nur aufgrund der Volumenänderung und der Kristallisation zu. Da keine gesicherten Erkenntnisse vorliegen, wie lange am Tropfenrand Partikel aufgesammelt werden, liegt bei der Wahl des Partikelvolumenanteils, bei dem umgeschaltet wird, eine Quelle der Unsicherheit und der Beeinflussung durch das mathematische Modell. Abb. 8.27 zeigt die Partikelanzahldichteverteilung im Tropfen am Ende des 1. Trocknungsabschnitts für einen Suspensionstropfen. Die Dicke der sich ausbildenden Hülle hängt deutlich vom Volumenanteil ab, bei dem eine Zelle keine Partikel mehr aufsammelt. Der Einfluß der Diskretisierung im Falle der Kristallisation ist jedoch geringer, weil hier die Verteilung des Massenanteils der gelösten Substanz einen größeren Einfluß hat (Abb. 8.28).
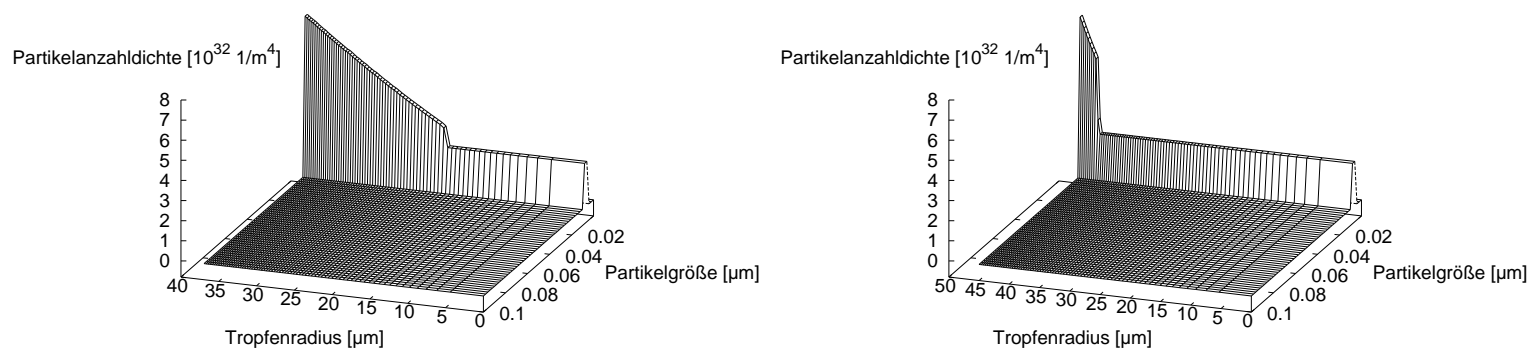

Abbildung 8.27: Partikelanzahldichteverteilung über dem Tropfenradius und der Partikelgröße in Suspensionstropfen am Ende des 1. Trocknungsabschnitts, links: Abbruch des Aufsammelns bei 30\%, rechts: Abbruch des Aufsammelns bei 55\%
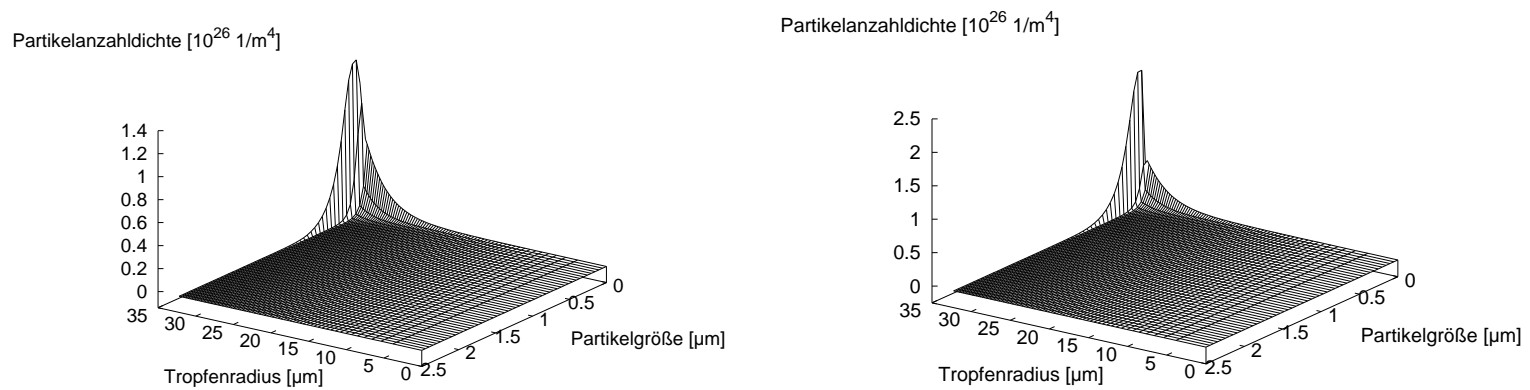

Abbildung 8.28: Partikelanzahldichteverteilung in Kaliumsulfatlösungstropfen am Ende des 1. Trocknungsabschnitts, links: Abbruch des Aufsammelns bei 30\%, rechts: Abbruch des Aufsammelns bei $55 \%$ 


\section{Kapitel 9}

\section{Zusammenfassung und Ausblick}

Die Sprühtrocknung ist ein seit etwa 100 Jahren bekannter und in vielen Industrien eingesetzter Prozeß zur Herstellung von Pulvern, doch beruhen Steuerung und Auslegung noch immer vorwiegend auf empirisch gewonnenen Erkenntnissen und Erfahrungen. Um ein tiefergehendes Verständnis der Vorgänge während der Sprühtrocknung zu gewinnen, ist im Rahmen der Arbeit ein Modell zur Beschreibung von Feststoffbildungsvorgängen in Einzeltropfen bei der Sprühtrocknung entwickelt worden. In den letzten Jahren hat sich eine Reihe von Arbeiten mit dem Trocknungsprozeß von Einzeltropfen bei gleichzeitiger Feststoffbildung beschäftigt. Die meisten dieser in den letzten Jahren entwickelten Modelle beschreiben die sich bildende Feststoffstruktur jedoch nur sehr oberflächlich. Die Dicke der Feststoffhülle, die sich bei der Sprühtrocknung in den meisten Fällen ausbildet, wird in der Regel aus einer Massenbilanz berechnet und die Hülle dann lediglich als Widerstand in der Energie- und Massenbilanz berücksichtigt. Zur Beschreibung der Pulvereigenschaften ist jedoch die detaillierte Kenntnis der mikroskopischen Partikeleigenschaften notwendig. Dazu müssen die Verteilung des Feststoffs im Tropfen sowie weitere Eigenschaften wie die Größe der mikroskopischen Feststoffpartikel im Tropfen vorausberechnet werden. Aus der Feststoffverteilung ergibt sich dann auch die Struktur der festen Hülle.

Das Hauptanliegen dieser Arbeit ist, ein besseres Verständnis der mikroskopischen, physikalischen Vorgänge während der Trocknung eines Lösungs- oder Suspensionstropfens zu gewinnen. Dazu ist ein eindimensionales Modell entwickelt worden, das die Feststoffbildung mit Populationsbilanzen beschreibt. Mit den so berechneten Partikelanzahldichteverteilungen kann die Struktur der sich während des Trocknungsprozesses ausbildenden Feststoffhülle beschrieben werden. Die Populationsbilanzen beschreiben im Gegensatz zu bestehenden Modellen neben der Größe der entstehenden Partikel auch ihre Lage im Raum, d.h. sie haben zwei Dimensionen. Beim Schrumpfungsprozeß werden die Partikel am Tropfenrand durch die zurückweichende Phasengrenze mit transportiert. Zur Beschreibung dieses Vorgangs ist erstmals 
eine Randbedingung entwickelt worden. Durch die Verwendung von Populationsbilanzen zur Beschreibung der Feststoffbildung ist es im Gegensatz zu bisherigen Modellen darüber hinaus möglich, auch die Trocknung von Suspensionstropfen mit demselben Modell zu simulieren. Die Populationsbilanzen werden mit der Energie- und Massenbilanz gekoppelt gelöst, so daßs im Tropfen die sich ändernde radiale Verteilung des Massenanteils der gelösten Substanz und die Temperaturverteilung berechnet werden können. Im Gegensatz zu bisherigen Arbeiten wird darüber hinaus bei der Diffusion berücksichtigt, daß die beiden Komponenten der Lösung stark unterschiedliche Dichten haben und dadurch ein konvektiver Ausgleichsstrom berücksichtigt werden muß, weil die durch Diffusion hervorgerufenen Massenströme im Tropfen unterschiedlich groß sind. Der Wärmetransport im Tropfen erfolgt sowohl durch Leitung als auch durch den Massentransport im Tropfen. Alle Gleichungen werden in einem normierten Koordinatensystem gelöst, um die Schrumpfung des Tropfens simulieren zu können. Die hyperbolischen Populationsbilanzen werden mit einem semidiskreten Finite-Volumen-Verfahren und die diffusionsdominierte Massen- und Energiebilanz mit einem semidiskreten ZentraleDifferenzen-Verfahren gekoppelt gelöst. Auf diese Weise können Standardlösungsverfahren zur Lösung des Gleichungssystems verwendet werden. Um das Herausfallen der Partikel aus dem Tropfen zu verhindern, ist das Diskretisierungsverfahren von Pennington und Berzins [58] am Tropfenrand modifiziert worden.

Es sind Modellrechnungen mit unterschiedlichen Parametereinstellungen durchgeführt worden, um deren Einfluß auf die Partikelanzahldichteverteilung im Tropfen zu untersuchen. Der Schwerpunkt der Untersuchungen liegt dabei zum einen auf der radialen Verteilung der Partikel im Tropfen und zum anderen ihrer Größe in Abhängigkeit von den Prozeßparametern. Dabei zeigte sich, daß die Größenverteilung der sich im Tropfen bildenden Partikel stark von der Schrumpfungsgeschwindigkeit des Tropfens abhängt. Bei langsamem Trocknen entstehen wenige große Partikel, während bei schnellem Trocknen viele kleine Partikel entstehen. Das Aufsammeln der innen liegenden Partikel durch die sich bewegende Phasengrenze führt dazu, daß die Partikelanzahldichteverteilung am Tropfenrand grundsätzlich höher ist als weiter innen. Es entstehen also wie auch experimentell beobachtet bevorzugt Hohlpartikel. Bei langsamem Trocknen entstehen jedoch auch im Inneren mehr Partikel. Dies ist in Übereinstimmung mit experimentellen Untersuchungen und bestehenden Modellen. Bei den Rechnungen hat sich auch gezeigt, daß der Temperaturgradient im Tropfen während des Trocknungsprozesses sehr gering ist, weil die Wärmeleitung im Tropfen sehr viel schneller ist als die Wärmeübertragung vom umgebenden Trocknungsgas in den Tropfen. Da dem Modell keine Informationen über die zeitliche Änderung der Umgebungsbedingungen im Trocknungsraum während des Trocknungsprozesses zur Verfügung stehen, sind die Rechnungen mit konstanten Umgebungsbedingungen durchgeführt worden. Um den Einfluß der sich im Laufe des Trocknungspro- 
zesses verändernden Eigenschaften des Trocknungsgases zu berücksichtigen, sind zusätzlich Rechnungen durchgeführt worden, bei denen die abnehmende Temperatur und der zunehmende Wasserdampfgehalt des Trocknungsgases berücksichtigt worden sind. Dabei zeigt sich, daß durch den verlangsamten Trocknungsprozeß die Partikelmorphologie deutlich von der abweicht, die mit konstanten Umgebungsbedingungen berechnet worden ist.

Da sich die bisherigen Arbeiten nicht mit der detaillierte Beschreibung des Hüllenwachstums befassen, sind zwei verschiedene mathematische Ansätze untersucht worden, die diesen Vorgang beschreiben können: Die Hülle kann durch ein weiteres äußeres Koordinatensystem oder durch das Strecken der äußeren Stützstellen, in denen sich ein bestimmter Volumenanteil an Partikeln befindet, beschrieben werden. Der zweite Ansatz hat sich dabei als vielversprechend herausgestellt. Mit diesem Ansatz muß als nächster Schritt das Wachsen der Hülle simuliert werden. Zur Simulation des Aufblähens der Hülle und ihrer Festigkeit ist ebenfalls ein Modell entwickelt worden, das noch in das Modell integriert werden muß. Das Tropfenmodell bietet darüber hinaus die Möglichkeit, statt der Kristallisation auch die Polymerisation als Feststoffbildungsmechanismus zu verwenden, indem Polymerisationsreaktionen als Feststoffbildungsmechanismus integriert werden. Die im Modell verwendete Ficksche Diffusion kann auch durch die Simulation von Mehrstoffgemischen ersetzt werden, um die im industriellen Alltag in der Regel vorkommenden Vielstoffgemische simulieren zu können. So können während des Trocknens auftretende Entmischungsvorgänge simuliert werden.

Zur Validierung des Modells sind experimentelle Untersuchungen durchgeführt worden. Es zeigte sich jedoch, daß leistungsfähigere Versuchsanlagen für eine erfolgreiche Validierung notwendig sind. Zu diesem Zweck ist ein zuverlässiger Tropfengenerator zur Erzeugung monodisperser Tropfen entwickelt worden, der für eine zukünftige Validierung eingesetzt werden und mit kostengünstiger Steuerungselektronik betrieben werden kann. Versuche haben gezeigt, daß der Tropfengenerator beim Betrieb im Bereich der theoretisch ermittelten optimalen Frequenzen monodisperse Tropfen produziert. In einem Fallturm, der höhere Temperaturen erreicht als der verwendete, müssen dann die Tropfen bei verschiedenen Parametereinstellungen getrocknet werden, so daß die Endstruktur der Partikel in Abhängigkeit von definierten Anfangs- und Randbedingungen untersucht werden kann. Weitere Möglichkeiten wären die Untersuchung von Lösungstropfen in einem Levitator oder von Tropfen am Draht, wobei hochauflösende Kameras zur Dokumentation des Trocknungsprozesses notwendig sind. 


\section{Anhang A}

\section{Programmstruktur}

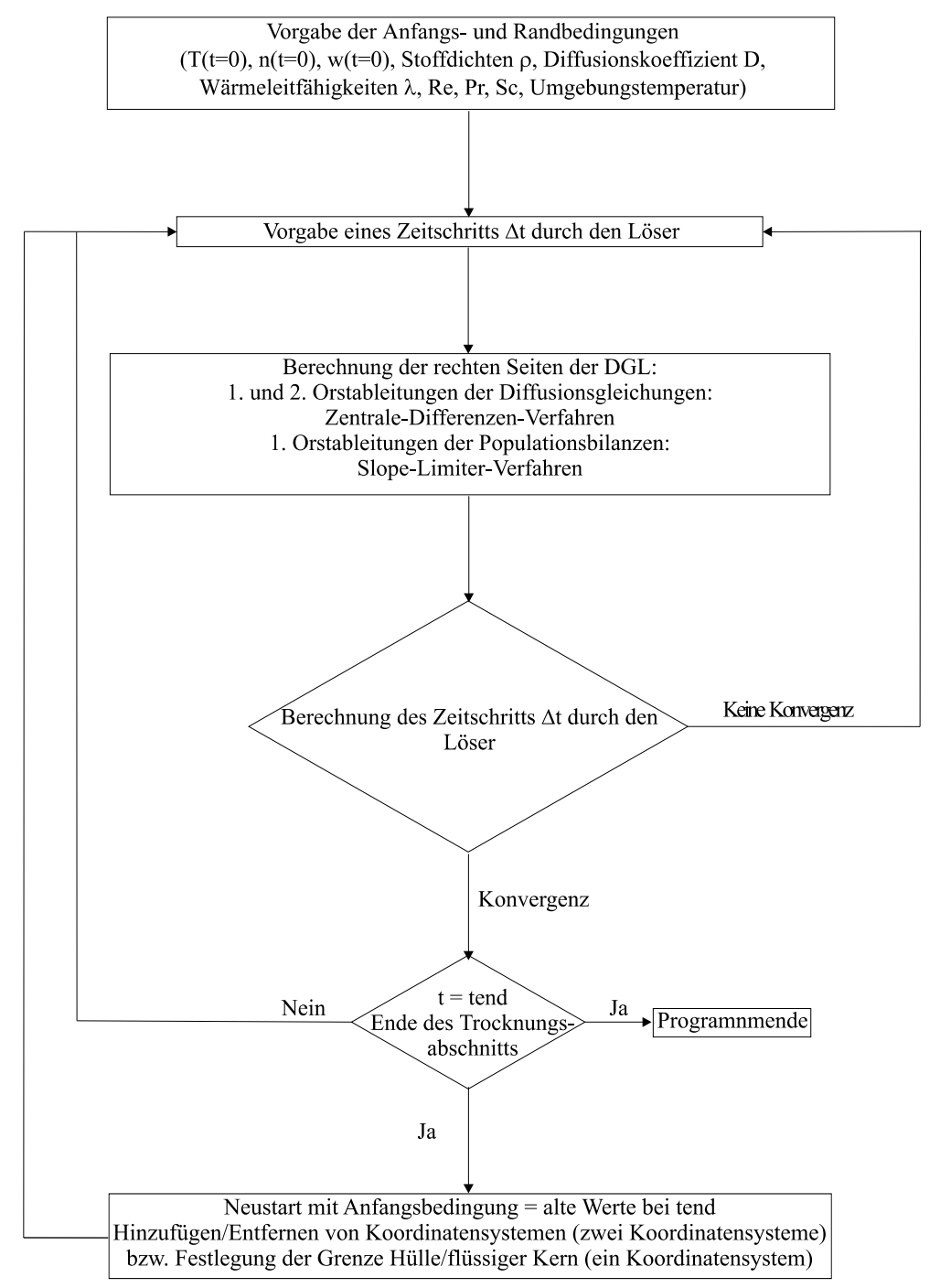

Abbildung A.1: Struktur des Programms 


\section{Literaturverzeichnis}

[1] FeIse, H. J.: Gedanken zur Zukunft der Feststoffverfahrenstechnik. Chemie Ingenieur Technik, 74(12):1661-1666, 2002.

[2] SChubert, H.: Handbuch der Mechanischen Verfahrenstechnik (Band 1). WILEY-VCH Verlag, 2003.

[3] Masters, K.: Spray drying in practice. SprayDryConsult International ApS, 2002.

[4] Huntington, D. H.: The influence of spray drying process on product properties. Drying Technology, 22(6):1261-1287, 2004.

[5] Lefebvre, A. H.: Atomization and sprays. Hemisphere Publishing Corporation, 1989.

[6] Deng, Z.-T. und S.-M. Jeng: Numerical simulation of droplet deformation in convective flows. AIAA Journal, 30(5):1290-1297, 1992.

[7] Haywood, R. J., M. Renksizbulut und G. D. Raithby: Transient deformation and evaporation of droplets at intermediate Reynolds numbers. Int. J. Heat Mass Transfer, 37(9):1401-1409, 1994.

[8] Frohn, A. und N. Roth: Dynamics of droplets. Springer-Verlag, Berlin, 1. Auflage, 2000

[9] Verdurmen, R. E. M., P. Menn, J. Ritzert, S. Blei, G. C. S. Nhumaio, T. Sonne Sorensen, M. Gunsing, J. Straatsma, M. Verschueren, M. Sibeijn, G. Schulte, U. Fritsching, K. Bauckhage, C. Tropea, M. Sommerfeld, A. P. Watkins, A. J. Yule und H. Schonfeldt: Simulation of agglomeration in spray drying installations: The EDECAD project. Drying Technology, 22(6):1403-1461, 2004.

[10] Chung, N. J.: The motion of partcles inside a droplet. Transactions of the ASME, 104:438-445, 1982.

[11] Chen, W.-H.: Unsteady absorption of sulfur dioxide by an atmospheric water droplet with internal circulation. Atmospheric Environment, 35:2375-2393, 2001. 
[12] Renksizbulut, M. und R. J. Haywood: Transient Droplet Evaporation with Varaiable Properties ans Internal Circulation at Intermediate Reynolds Numbers. Int. J. Multiphase Flow, 14(2):189-202, 1988.

[13] Gogos, G. und P. S. Ayyaswamy: A model for the evaporation of a slowly moving droplet. Combustion and Flame, 74:111-129, 1988.

[14] Bhatia, R. und W. A. Sirignano: Transient heating and burning of droplet containing a single metal particle. Combust. Sci. and Tech., 84:141-161, 1992.

[15] Bhatia, R. und W. A. SiRignano: Convective burning of a droplet containing a single metal particle. Combustion and Flame, 93:215-229, 1993.

[16] Jog, M. A., P. S. Ayyaswamy und I. M. Cohen: Evaporation and combustion of a slowly moving liquid fuel droplet: higher-order theory. J. Fluid Mech., 307:135-165, 1996.

[17] Pagel, S. und G. P. Merker: Modellierung der Tropfenverdampfung eines Mehrkomponentenbrennstoffs mit detaillierter Betrachtung des Tropfeninneren. Chemie Ingenieur Technik, 75(7):888-891, 2003.

[18] Sano, Y. und R. B. KeEY: The Drying of a spherical particle containing coloidal material into a hollow sphere. Chemical Engineering Science, 37(6):881-889, 1982.

[19] Cheong, H. W., G. V. Jeffreys und C.J.Mumford: A receding interface model for the drying of slurry droplets. A.I.Ch.E. Journal, 32(6):1334-1346, 1986.

[20] Nes̆ı́́, S. und J. VodniK: Kinetics of Droplet Evaporation. Chemical Engineering Science, 45(2):527-537, 1991.

[21] LEE, A. und C. K. LAW: Gasification and shell characteristics in slurry droplet burning. Combustion And Flame, 85:77-93, 1991.

[22] Bhatia, R. und W. A. Sirignano: Liquid vaporization from fine metal slurry droplets. American Institute of Aeronautics and Astronautics, Seiten 235-262, 1992.

[23] Elperin, T. und B. Krasovitov: Evaporation of liquid droplets containing small solid particles. International Journal of Heat Mass Transfer, 38(12):2259-2267, 1995.

[24] FORD, I. J.: Models of crystallization in evaporating droplets. Chemical Engineering Science, 1995.

[25] Hecht, J. P. und C. J. King: Spray drying: Influence of developing drop morphology on drying rates and retention of volatile substances. 2. Modeling. In. Eng. Chem. Res., 39:1766-1774, 2000. 
[26] Liang, H., K. Shinohara, H. Minoshima und K. Matsushima: Analysis of constant rate period of spray drying of slurry. Chemical Engineering Science, 56(6):2205-2213, 2001.

[27] Brenn, G., T. Wiedemann, D. Rensink, O. Kastner und A. L. Yarin: Modellierung und experimentelle Untersuchung der Morphologie sprühgetrockneter Partikel. Chemie Ingenieur Technik, 73(5):491-494, 2001.

[28] WeISS, C.: Modellierung und Simulation der Kristallisation in dispersen Systemen. Doktorarbeit, Universität Dortmund, 2002.

[29] Kadja, M. und G. Bergeles: Modelling of a slurry droplet drying. Applied Thermal Engineering, 23:829-844, 2003.

[30] BREnN, G.: Konzentrationsverteilungen in trocknenden Tropfen. Chemie Ingenieur Technik, 76(3):267-272, 2004.

[31] Chen, X. D. und S. X. Q. Lin: Air drying of milk droplet under constant and timedependent conditions. AIChE Journal, 51:1790-1799, 2005.

[32] Verkoeijen, D., G. A. Pouw, H. Meesters G. M und B. Scarlett: Population balances for particulate processes - a volume approach. Chemical Engineering Science, 57:2287-2303, 2002.

[33] Paschedag, A.: Kopplung zwischen Populationsbilanzen und CFD am Beispiel von Fällungsprozessen. Chemie Ingenieur Technik, 75(12):1835-1839, 2003.

[34] Campos, F. B. und P. L. C. LAGE: A numerical method for solving the transient multidimensional population balance equation using an Euler-Lagrange formulation. Chemical Engineering Science, 2002.

[35] Jeschar, R., R. Alt und E. Specht: Grundlagen der Wärmeübertragung. ViolaJeschar-Verlag, Goslar, 3. Auflage, 1990.

[36] Krischer, O. und W. Kast: Die wissenschaftlichen Grundlagen der Trocknungstechnik. Springer-Verlag, Berlin, 3. Auflage, 1992.

[37] Poling, B. E., J. M. Prausnitz und J. P. O'Connell: The properties of gases and liquids. McGraw-Hill, 5. Auflage, 2000.

[38] Charlesworth, D. H. und W. R. Marshall: Evaporation from drops containing dissolved solids. A.I.Ch.E. Journal, 6(1):9-23, 1960. 
[39] BÜtTIKeR, R.: Mechanism of particle formation during drying of free-falling drops containing solids. Ger.Chem. Eng., 4:298-304, 1981.

[40] Prandtl, L., K. Oswatitsch und K. Wieghardt: Führer durch die Strömungslehre. Springer-Verlag, Berlin, 9. Auflage, 1990.

[41] Mersmann, A.: Crystallization Technology Handbook. Marcel Dekker, Inc., 1. Auflage, 1995.

[42] Randolph, A. und M. Larson: Theory of particulate processes. Academic Press, 1988.

[43] Crank, J.: Free and moving boundary problems. Clarendon Press, Oxford, 1987.

[44] Bird, R. Byron, W. E. Stewart und E. N. Lightfoot: Transport Phenomena. New York: John Wiley \& Sons, 2. Auflage, 2002.

[45] Gidaspow, D.: Multiphase flow and fluidization. Academic Press, Boston, 1994.

[46] Fitzer, E., W. Fritz und G. Emig: Technische Chemie. Springer-Verlag, Berlin, 4. Auflage, 1989.

[47] Lin, J.-C. und J. W. Gentry: Spray drying drop morphology: Experimental study. Aerosol Science and Technology, 37(1):15-32, 2003.

[48] Atkins, A.: Physical Chemistry. VCH Verlagsgesellschaft mbH, 2. Auflage, 1990.

[49] Koch, M. und P. Walzel: Migration of solutes in drying suspension droplets. In: Spray Drying '01 and related processes, Dortmund, 2001.

[50] Beitz, W. und K.-H. KÜttner (Herausgeber): Dubbel. Taschenbuch für den Maschinenbau. Springer-Verlag, Berlin, 17. Auflage, 1990.

[51] Leschonski, K.: Vorlesungsskript: Mechanische Verfahrenstechnik. TU Clausthal, 1997.

[52] Sincovec, R. F. und N. M. Madsen: Software for nonlinear partial differential equations. ACM Transactions on mathematical software, 1(3):261-263, 1975.

[53] Christofides, P. D: Model-based control of particulate processes. Kluwer Academic Publishers, Dordrecht, 1. Auflage, 2002.

[54] Ewing, R. E. und H. WAng: A summary of numerical methods for time-dependent advection-dominated partial differential equations. Journal of Computational and Applied Mathematics, 128:423-445, 2001. 
[55] MalchereK, A.: Vorlesungsgkript: Numerische Methoden der Strömungsmechanik. Bundesanstalt für Wasserbau, 5.6 Auflage.

[56] Mantzaris, N. V., P. Daoutidis und F. SRienc: Numerical Simulation of multivariable cell population balance models. II. Spectral methods. Computers and Chemical Engineering, 25:1441-1462, 2001.

[57] Mantzaris, N. V., P. Daoutidis und F. SRienc: Numerical Simulation of multivariable cell population balance models. III. Finite Element methods. Computers and Chemical Engineering, 25:1463-1481, 2001.

[58] Pennigton, S. V. und M. Berzins: New (NAG) library software for first-order partial differential equations. ACM Transactions on mathematical software, 20(1):63-99, 1994.

[59] Wulkow, M., A. Gerstlauer und U. Nieken: Modeling and simulation of crystallization processes using parsival. Chemical Engineering Science, 56:2575-2588, 2001.

[60] Abdulle, A.: Fourth order Chebyshev methods with recurrence relation. SIAM Journal on Scientific Computing, 23(6):2041-2054, 2002.

[61] Abdulle, A. und A. Medovikov: Second order Chebyshev methods based on orthogonal polynomials. Numerische Mathematik, 90(1):1-18, 2001.

[62] Hairer, E. und G. WANneR: Solving ordinary differential equations II. Stiff and differential-algebraic problems. Springer Series in Computational Mathematics 14. Springer-Verlag, Berlin, 2. Auflage, 1996.

[63] Faires, J. D. und R. L. BuRden: Numerische Mathematik. Spektrum Akademischer Verlag, 1. Auflage, 1994.

[64] Taniguchi, I., T. Inoue und K. Asano: Evaporation of a salt water drop witg crystallization. Atomization and Sprays, 9:69-85, 1999.

[65] Walton, D. E. und C. J. Mumford: The morphology of spray-dried particles. The effect of process variables upon the morphology of spray-dried particles. Trans IChemE., 77(Part A):442-460, 1999.

[66] WAHL, B.: Über den Trocknungsverlauf frei fallender feststoffhaltiger Tropfen. Chemie Ingenieur Technik, 46(8):351, 1974.

[67] Yarin, A. L., G. Brenn und D. Rensink: Evaporation of acoustically levitated droplets of binary liquid mixtures. Hea and Fluid Flow, 23:471-486, 2002. 
[68] Brenn, G., O. Kastner, O. Rensink und C. Tropea: Evaporation and drying of multicomponent and multiphase droplets in a tube levitator. ILASS-Europe-99, 1999.

[69] Yang, J. C., W. Chien, M. King und W. L. Grosshandler: A simple piezoelectric droplet generator. Experiments in Fluids, 23:445-447, 1997.

[70] Walzel, P.: Zerstäuben von Flüssigkeiten. Chemie Ingenieur Technik, 62(12):983-994, 1990.

[71] Walzel, P., F. Schmelz und S. Schneider: Herstellen monodisperser Tropfen mit pneumatischen Ziehdüsen. Chemie Ingenieur Technik, 73(12):1599-1602, 2001.

[72] VDI-Wärmeatlas. Springer-Verlag, Berlin, 8. Auflage, 1997.

[73] Tavare, N. S. und M. R. Chivate: Analysis of batch evaporative ctystallizers. The Chemical Engineering Journal, 14:175-180, 1977.

[74] Römpp Online. Stuttgart: Thieme Verlag, 2005.

[75] Mersmann, A., B. Braun und M. Löffelmann: Prediction of crystallization coefficients of the population balance. Chemical Engineering Science, 57:4267-4275, 2002. 


\section{Danksagung}

Herrn Professor Weidner möchte ich für die Übernahme der Betreuung meiner Promotion danken. Herrn Professor Kümmel und Jürgen Bertling möchte ich dafür danken, daß sie mir das Thema zur Bearbeitung zur Verfügung gestellt haben. Ganz besonders möchte ich Jan Blömer für die vielen fruchtbaren Diskussionen und Ratschläge danken, ohne die ich diese Arbeit nicht erfolgreich beendet hätte. Dabei war er praktisch jederzeit zu einer Diskussion bereit. Torsten Hennig möchte ich für die intensive Unterstützung bei mathematischen und numerischen Fragen danken. Außerdem möchte ich allen am Fraunhofer Institut UMSICHT danken, die zum Gelingen der Arbeit beigetragen haben. Ganz besonders möchte ich mich auch bei meinen Eltern bedanken, ohne deren Unterstützung ich die Promotion nicht erfolgreich abgeschlossen hätte. 


\section{Lebenslauf}

$\begin{array}{ll}\text { Name: } & \text { Seydel } \\ \text { Vorname: } & \text { Philipp } \\ \text { Geburtstag: } & 18.12 .1973 \\ \text { Geburtsort: } & \text { Kiel } \\ \text { Eltern: } & \text { Dr. med. Hiltrud Seydel } \\ & \text { Prof. Dr. Ulrich Seydel }\end{array}$

\section{Schulausbildung}

$\begin{array}{ll}1980-1984 & \text { Grundschule in Bad Segeberg } \\ 1984-1993 & \text { Gymnasium in Bad Segeberg (Dahlmannschule) } \\ 18.06 .1993 & \text { Abitur }\end{array}$

Wehr- / Zivildienst

01.07.1993 - 18.01.1994 Ableistung des Wehrdienst

18.01.1994 - 30.09.1994 Ableistung des Zivildienst (Pflegedienst im Kreiskrankenhaus in Bad Segeberg)

\section{Universitätsausbildung}

$10.1994-03.2000$

27.03 .1997

12.04.1999-08.10.1999

22.03 .2000
Studium des Chemieingenieurwesens an der TU Clausthal Vordiplom im Fach Chemieingenieurwesen

Diplomarbeit bei der Firma Henkel KGaA in Düsseldorf Thema: Experimentelle Ermittlung und Modellierung der Druckabhängigkeit einer Guerbet-Reaktion

Diplom im Fach Chemieingenieurwesen

\section{Beruflicher Werdegang}

01.09.2000 - 30.09.2001 Prozeßingenieur bei der Firma Mattson Wet Products GmbH in Pliezhausen

01.10.2001 - 30.06.2005 Wissenschaftlicher Mitarbeiter am Fraunhofer Institut für Umwelt-, Sicherheits- und Energietechnik in Oberhausen 